\title{
Wigner Quasiprobability with an Application to Coherent Phase States
}

\author{
Alfred Wünsche \\ Institut für Physik, Humboldt University, Berlin, Germany \\ Email: alfred.wuensche@physik.hu-berlin.de
}

How to cite this paper: Wünsche, A. (2018) Wigner Quasiprobability with an Application to Coherent Phase States. $A d$ vances in Pure Mathematics, 8, 564-614. https://doi.org/10.4236/apm.2018.86034

Received: April 16, 2018

Accepted: June 25, 2018

Published: June 28, 2018

Copyright $\odot 2018$ by author and Scientific Research Publishing Inc. This work is licensed under the Creative Commons Attribution International License (CC BY 4.0).

http://creativecommons.org/licenses/by/4.0/

c) (i) Open Access

\begin{abstract}
Starting from Wigner's definition of the function named now after him we systematically develop different representation of this quasiprobability with emphasis on symmetric representations concerning the canonical variables $(q, p)$ of phase space and using the known relation to the parity operator. One of the representations is by means of the Laguerre $2 \mathrm{D}$ polynomials which is particularly effective in quantum optics. For the coherent states we show that their Fourier transforms are again coherent states. We calculate the Wigner quasiprobability to the eigenstates of a particle in a square well with infinitely high impenetrable walls which is not smooth in the spatial coordinate and vanishes outside the wall boundaries. It is not well suited for the calculation of expectation values. A great place takes on the calculation of the Wigner quasiprobability for coherent phase states in quantum optics which is essentially new. We show that an unorthodox entire function plays there a role in most formulae which makes all calculations difficult. The Wigner quasiprobability for coherent phase states is calculated and graphically represented but due to the involved unorthodox function it may be considered only as illustration and is not suited for the calculation of expectation values. By another approach via the number representation of the states and using the recently developed summation formula by means of Generalized Eulerian numbers it becomes possible to calculate in approximations with good convergence the basic expectation values, in particular, the basic uncertainties which are additionally represented in graphics. Both considered examples, the square well and the coherent phase states, belong to systems with $S U(1,1)$ symmetry with the same index $k=\frac{1}{2}$ of unitary irreducible representations.
\end{abstract}

\section{Keywords}

Parity Operator, Quantum Square Well, Coherent States, $S U(1,1)$ Group and Realizations, Glauber-Sudarshan and Husimi-Kano Quasiprobability, 
London Phase States, Phase Distribution, Unorthodox Entire Function,

Laguerre 2D Polynomials, Generalized Eulerian Numbers

\section{Introduction}

Most representations of probability theory begin with the discussion of some examples where probabilities play a main role and introduce then axiomatically the probability as a positively semi-definite and "normalized" function over a set of events. The main purpose of this function is to allow us to calculate mean values and their variances or more generally expectation values for arbitrary functions over the set of events when the initial conditions or the prehistory of the events are not fully under control of the experimenter or observer. The results of such calculations are then true in the mean for great ensembles of "equal" events and of their dynamics which are made under the same uncontrolled or uncontrollable initial conditions. In Hamilton dynamics of a system of one degree of freedom a trajectory is fully determined by a pair $(q, p)$ of canonical coordinate and momentum in two-dimensional phase space as initial condition and the probability function is given by a positive semi-definite function $F(q, p)$ (called distribution or partition function) and from the dynamics of single trajectories, in principle, can be determined by the time evolution of the function $F(q, p)$ (the lately discovered cases of possible chaotic dynamics destroy this assumption in some way). After foundation of probability theory in 17-th century mainly by Blaise Pascal and great contributions by Jakob B. 1654-1705 and Daniel (I.) Bernoulli 1700-1782 and by Huygens in 17-th century and by Laplace and Bayes in 18-th century a culminating point was reached by the axiomatic foundation which in the now finally accepted form was given in 1933 by Kolmogorov.

Quantum mechanics gave birth to a new kind of probabilities which are not definitely non-negative and which are called quasiprobabilities. The first and, for some reason, forever the most important such quasiprobability is the Wigner quasiprobability $W(q, p)$ [1] (see also [2]), often called Wigner function. Due to substitution of the classical canonical variables $(q, p)$ by non-commuting operators $(Q, P)$ (commutator $[Q, P]=\mathrm{i} \hbar I ; I$ identity operator in representation

space; $\hbar \equiv \frac{h}{2 \pi}, h$ Planck's constant) in quantum mechanics a single event is not determined as in classical theory by a (displaced) delta function $F(q, p)=\delta\left(q-q_{0}\right) \delta\left(p-p_{0}\right)$ and its trajectory in phase space is not defined and one may best have moving "spots" of events with minimal uncertainty products $\Delta q \Delta p=\hbar / 2$ equivalent to Gaussian distribution functions with $\Delta q=\Delta p=\sqrt{\hbar / 2}$

$$
F(q, p)=\frac{1}{\pi \hbar} \exp \left\{-\frac{\left(q-q_{0}\right)^{2}+\left(p-p_{0}\right)^{2}}{\hbar}\right\}, \quad \int \mathrm{d} q \wedge \mathrm{d} p F(q, p)=1,
$$


with all possible points (displacements $q_{0}$ and $p_{0}$ ) in phase space. The functions $F(q, p)$ to different displacements $\left(q_{0}, p_{0}\right)$ are overlapping and, therefore, are not independent from each other. The canonical operators $(Q, P)$ are determined up to unitary transformations which preserve the commutation relations and correspond to canonical transformations in classical theory [3].

Expectation values cannot only be calculated by probability or distribution functions $F(q, p)$ but also from all of their transforms which can be uniquely inverted, for example, with the Fourier transform or with the Radon transform of $F(q, p)$ or also from the wave functions. An important criterion for a probability function $F(q, p)$ is that a displacement of the state in the phase plane displaces the arguments $(q, p)$ in corresponding way. In Section 4 we calculate the Wigner quasiprobability for a particle in a square well with infinitely high impenetrable walls. This example provides a Wigner quasiprobability which for the spatial coordinate $q$ vanishes outside the wall borders and therefore is not smooth (infinitely differentiable with continuous derivatives) at the wall borders. Due to the quadratic energy spectrum it possesses internal $S U(1,1)$-symmetry. Both properties are equivalent [4]. In Section 5 we make the transition from canonical operators to pairs of boson annihilation and creation operators (and, correspondingly, complex coordinates) that is important for quantum optics, e.g., [5]-[10]. The coherent states are here a very effective mean for the treatment of many problems but it is less known that the Fourier transformation of coherent states provides anew coherent states (Section 6 and Appendix A). A further effective mean for the short representation of many basic relations of quantum optics is the use of Laguerre 2D polynomials which we dealt with in some papers (see [11] and references therein) which is shortly touched in Section 7. From Section 8 on we prepare the calculation of the Wigner quasiprobability for coherent phase states and which similar to states in an infinitely high square well (Section 4) possess internal $S U(1,1)$-symmetry (Perelomov [12] [13] [14]). The difficulty is here that a very unorthodox entire function is involved (Appendix D). In the paper [15] the authors claim that they calculate the Wigner function for a phase state but they make this for a non-normalizable London phase state and the calculated function is properly speaking not a Wigner function and is non-normalizable (Sections 8-11). Due to the presence of the mentioned unorthodox entire function which is difficult to manage the Wigner quasiprobability of a coherent phase state is less appropriate for the calculation of expectation values and variances. We discuss in addition in Sections 12-14 another possibility by means of Generalized Eulerian numbers, which we represented in a recent publication [16] (see also [17] [18]).

\section{The Wigner Quasiprobability in Different Representations and Their Equivalence}

In quantum mechanics it is not possible to find a probability function $F(q, p)$ 
over the phase space which satisfies all requirements for such functions from classical theory for the calculation of expectation values, in particular, positive semi-definiteness. One also cannot translate in a unique way a classical function $A(q, p)$ over the phase space $(q, p)$ into a quantum-mechanical function of the operators $A(Q, P)$ since such a function is ill-defined without giving a rule for the ordering of the non-commuting operators $(Q, P)$ in this function. Thus one has two problems, first to give an ordering rule for operator functions $A(Q, P)$ which substitute classical functions $A(q, p)$ and then to find a quasidistribution function which allows to calculate all expectation values of well-defined operator functions and which should be in best agreement with the classical distribution function. This is only possible by compromises.

The functions $|\psi\rangle \in \mathcal{H}$ of the irreducible representation of the Heisenberg-Weyl (Lie) algebra of the canonical commutation relations $[Q, P]=\mathrm{i} \hbar I,\left(Q=Q^{\dagger}, P=P^{\dagger}\right)$ in an infinite-dimensional Hilbert space $\mathcal{H}$ form the deeper level discovered by quantum mechanics to which we do not possess a direct access with macroscopic instruments and in almost all measurable expectation values we have it to do with the representation $\mathcal{H} \times \mathcal{H}^{*}$ of objects of combinations of $|\varphi\rangle\langle\psi|$ or, more generally, $|\varphi\rangle\langle\psi|$.

For functions $f(Q)$ of $Q$ or $f(P)$ of $P$ alone one does not have the ordering problem and expectation values of such operator functions for pure states $|\psi\rangle$ can be calculated with two (different!) normalized functions $W(q)$ or $W(p)$, correspondingly, according to

$$
\begin{aligned}
& \overline{f(Q)} \equiv\langle\psi|f(Q)| \psi\rangle=\int_{-\infty}^{+\infty} \mathrm{d} q W(q) f(q), \quad \int_{-\infty}^{+\infty} \mathrm{d} q W(q)=1, \\
& \overline{f(P)} \equiv\langle\psi|f(P)| \psi\rangle=\int_{-\infty}^{+\infty} \mathrm{d} p W(p) f(p), \quad \int_{-\infty}^{+\infty} \mathrm{d} p W(p)=1 .
\end{aligned}
$$

The two (different) functions $W(q)$ and $W(p)$ satisfy all requirements for genuine positively semi-definite probability densities.

Wigner, apparently, looked for a quasidistribution function $W(q, p)$ normalized according to

$$
\int_{-\infty}^{+\infty} \mathrm{d} q \int_{-\infty}^{+\infty} \mathrm{d} p W(q, p)=1,
$$

which integrated over one of the canonical variables $(q, p)$ possesses the property ( $\varrho$ density operator)

$$
W(q) \equiv \int_{-\infty}^{+\infty} \mathrm{d} p W(q, p)=\langle q|\varrho| q\rangle, \quad W(p) \equiv \int_{-\infty}^{+\infty} \mathrm{d} q W(q, p)=\langle p|\varrho| p\rangle,
$$

and which is the best compromise for such a function in quantum theory and found [1] [2]

\footnotetext{
${ }^{1}$ Wigner denotes it by $P(x, p)$ and generalizes it to $n$ dimensions

$(x, p) \rightarrow\left(x_{1}, \cdots, x_{n} ; p_{1}, \cdots, p_{n}\right) ; \frac{1}{\hbar \pi} \rightarrow \frac{1}{(\hbar \pi)^{n}}$. He remarks that it was found together with L. Szilard. We apply here Dirac's notation for states with $\psi(q) \equiv\langle q \mid \psi\rangle=\langle\psi \mid q\rangle^{*}$ and write it for density operators $\varrho$ with $\varrho \rightarrow|\psi\rangle\langle\psi|$ for pure states $|\psi\rangle$.
} 


$$
W(q, p)=\frac{1}{\pi \hbar} \int_{-\infty}^{+\infty} \mathrm{d} q_{0}\left\langle q-q_{0}|\varrho| q+q_{0}\right\rangle \exp \left(\mathrm{i} \frac{2 p q_{0}}{\hbar}\right), \quad(Q|q\rangle=q|q\rangle),
$$

where $|q\rangle$ are the eigenstates of the operator $Q$ to eigenvalues $q$. By Fourier transformation according to

$$
|p\rangle=\frac{1}{\sqrt{2 \pi \hbar}} \int_{-\infty}^{+\infty} \mathrm{d} q \exp \left(+\mathrm{i} \frac{p q}{\hbar}\right)|q\rangle, \quad|q\rangle=\frac{1}{\sqrt{2 \pi \hbar}} \int_{-\infty}^{+\infty} \mathrm{d} p \exp \left(-\mathrm{i} \frac{q p}{\hbar}\right)|p\rangle,
$$

one finds the analogous formula by the wave functions $\langle p \mid \psi\rangle$ in momentum representation

$$
W(q, p)=\frac{1}{\pi \hbar} \int_{-\infty}^{+\infty} \mathrm{d} p_{0}\left\langle p+p_{0}|\varrho| p-p_{0}\right\rangle \exp \left(\mathrm{i} \frac{2 q p_{0}}{\hbar}\right), \quad(P|p\rangle=p|p\rangle)
$$

In this transformation we used the scalar products

$$
\left\langle q^{\prime} \mid q\right\rangle=\delta\left(q-q^{\prime}\right),\left\langle p^{\prime} \mid p\right\rangle=\delta\left(p-p^{\prime}\right),\langle q \mid p\rangle=\frac{1}{\sqrt{2 \pi \hbar}} \exp \left(\mathrm{i} \frac{p q}{\hbar}\right)=\langle p \mid q\rangle^{*}
$$

and the completeness relations

$$
\int_{-\infty}^{+\infty} \mathrm{d} q|q\rangle\left\langle q\left|=\int_{-\infty}^{+\infty} \mathrm{d} p\right| p\right\rangle\langle p|=I .
$$

The definitions (2.4) and (2.6) are fully equivalent to the following definition $(\langle A\rangle \equiv \operatorname{Trace}(A)$ denotes the trace of an operator $A$ )

$$
W(q, p)=\left\langle\varrho \exp \left(-Q \frac{\partial}{\partial q}-P \frac{\partial}{\partial p}\right)\right\rangle \delta(q) \delta(p),
$$

showing explicitly the symmetry between the canonical variables $(q, p)$ in the definition of the Wigner quasiprobability. The equivalence of (2.9) to (2.4) and (2.6) can be proved, for example, as follows. Starting from (2.9) and using the well-known relation $\mathrm{e}^{A} \mathrm{e}^{B}=\mathrm{e}^{A+B+\frac{1}{2}[A, B]}$ if $A$ and $B$ commute with the commutator $[A, B]$, i.e. $[A,[A, B]]=[A,[A, B]]=0$, and the completeness of the states $|q\rangle$ according to (2.8), one finds

$$
\begin{aligned}
& \left\langle\rho \exp \left(-Q \frac{\partial}{\partial q}-P \frac{\partial}{\partial p}\right)\right\rangle \delta(q) \delta(p) \\
& =\left\langle\rho \exp \left(-\frac{Q}{2} \frac{\partial}{\partial q}\right) \exp \left(-P \frac{\partial}{\partial p}\right) \exp \left(-\frac{Q}{2} \frac{\partial}{\partial q}\right)\right\rangle \delta(q) \delta(p) \\
& =\int_{-\infty}^{+\infty} \mathrm{d} q^{\prime} \int_{-\infty}^{+\infty} \mathrm{d} q^{\prime \prime}\left\langle q^{\prime}|\varrho| q^{\prime \prime}\right\rangle\left\langle q^{\prime \prime}\left|\exp \left(-P \frac{\partial}{\partial p}\right)\right| q^{\prime}\right\rangle \exp \left(-\frac{q^{\prime}+q^{\prime \prime}}{2} \frac{\partial}{\partial q}\right) \delta(q) \delta(p) \\
& =\int_{-\infty}^{+\infty} \mathrm{d} q^{\prime} \int_{-\infty}^{+\infty} \mathrm{d} q^{\prime \prime}\left\langle q^{\prime}|\varrho| q^{\prime \prime}\right\rangle\left\langle q^{\prime \prime}\left|\exp \left(-P \frac{\partial}{\partial p}\right)\right| q^{\prime}\right\rangle \delta\left(q-\frac{q^{\prime}+q^{\prime \prime}}{2}\right) \delta(p) \\
& =2 \int_{-\infty}^{+\infty} \mathrm{d} q_{0}\left\langle q-q_{0}|\varrho| q+q_{0}\right\rangle\left\langle q+q_{0}\left|\exp \left(-P \frac{\partial}{\partial p}\right)\right| q-q_{0}\right\rangle \delta(p)
\end{aligned}
$$

where we made the substitution $q^{\prime}=q-q_{0}, q^{\prime \prime}=q+q_{0}$ with $\mathrm{d} q^{\prime} \wedge \mathrm{d} q^{\prime \prime}=2 \mathrm{~d} q \wedge \mathrm{d} q_{0}$ of the integration variables. In a last step using the completeness of the states $|p\rangle$ according to (2.8) and their scalar products $\langle q \mid p\rangle$ 
given in (2.7), one calculates

$$
\begin{aligned}
& \left\langle q+q_{0}\left|\exp \left(-P \frac{\partial}{\partial p}\right)\right| q-q_{0}\right\rangle \delta(p) \\
& =\int_{-\infty}^{+\infty} \mathrm{d} p^{\prime}\left\langle q+q_{0} \mid p^{\prime}\right\rangle\left\langle p^{\prime} \mid q-q_{0}\right\rangle \underbrace{\exp \left(-p^{\prime} \frac{\partial}{\partial p}\right) \delta(p)}_{\delta\left(p-p^{\prime}\right)} \\
& =\frac{1}{2 \pi \hbar} \exp \left(\mathrm{i} \frac{p\left(q+q_{0}\right)-p\left(q-q_{0}\right)}{\hbar}\right)=\frac{1}{2 \pi \hbar} \exp \left(\mathrm{i} \frac{2 p q_{0}}{\hbar}\right) .
\end{aligned}
$$

Thus the relation (2.10) in connection with (2.11) proves the equivalence of (2.9) to (2.4).

We now give a representation of the Wigner quasiprobability by the displaced parity operator. With respect to the complete sets of eigenstates $|q\rangle$ or $|p\rangle$ of the Hermitean operators $Q$ or $P$ the parity operator $\Pi$ acts according to

$$
\Pi|q\rangle=|-q\rangle, \quad \Pi|p\rangle=|-p\rangle,
$$

and the parity operator $\Pi$ itself can be represented by the following equivalent integrals

$$
\Pi=\int_{-\infty}^{+\infty} \mathrm{d} q|q\rangle\left\langle-q\left|=\int_{-\infty}^{+\infty} \mathrm{d} p\right| p\right\rangle\langle-p|=\Pi^{\dagger}=\Pi^{-1}, \quad \Pi^{2}=I .
$$

From this follows

$$
\Pi Q=-Q \Pi, \quad \Pi P=-P \Pi, \quad \Pi D(q, p)=-D(-q,-p) \Pi,
$$

and furthermore

$$
\Pi Q \Pi^{\dagger}=-Q, \quad \Pi P \Pi^{\dagger}=-P, \quad \Pi D(q, p) \Pi=-D(-q,-p),
$$

where $D(q, p)$ is the unitary displacement operator defined by ${ }^{2}$

$$
D(q, p) \equiv \exp \left(-\mathrm{i} \frac{q P-p Q}{\hbar}\right), \quad(D(q, p))^{-1}=D(-q,-p)=(D(q, p))^{\dagger} \text {. }
$$

Factorizations (or disentanglements) of the operator $D(q, p)$ are obtained by the already mentioned theorem used to derive Equation (2.10) and are

$$
\begin{aligned}
D(q, p) & =\exp \left(\mathrm{i} \frac{q p}{2 \hbar}\right) \exp \left(-\mathrm{i} \frac{q P}{\hbar}\right) \exp \left(\mathrm{i} \frac{p Q}{\hbar}\right) \\
& =\exp \left(-\mathrm{i} \frac{p q}{2 \hbar}\right) \exp \left(\mathrm{i} \frac{p Q}{\hbar}\right) \exp \left(-\mathrm{i} \frac{q P}{\hbar}\right), \quad D(0,0)=I .
\end{aligned}
$$

The product of two displacement operators is also a displacement operator (group property) multiplied by a phase factor

$$
D(q, p) D\left(q^{\prime}, p^{\prime}\right)=\exp \left(-\mathrm{i} \frac{q p^{\prime}-p q^{\prime}}{2 \hbar}\right) D\left(q+q^{\prime}, p+p^{\prime}\right),
$$

with the trace

$$
\left\langle D(q, p) D\left(q^{\prime}, p^{\prime}\right)\right\rangle=2 \pi \hbar \delta\left(q+q^{\prime}\right) \delta\left(p+p^{\prime}\right), \quad\langle D(q, p)\rangle=2 \pi \hbar \delta(q) \delta(p) .
$$

${ }^{2}$ Klauder and Sudarshan [6] (see also Klauder and Skagerstam [8]) denote it by $U(p, q)$ and the coherent states by $|z\rangle \equiv|p, q\rangle$. 
The displaced parity operator $\Pi(q, p)$ is defined as a Hermitean operator by

$$
\Pi(q, p) \equiv D(q, p) \Pi(D(q, p))^{\dagger}=(\Pi(q, p))^{\dagger} .
$$

Using (2.10), (2.18) and (2.19) and $\Pi^{2}=I$ one derives the following properties of the displaced parity operator

$$
\left\langle\Pi(q, p) \Pi\left(q^{\prime}, p^{\prime}\right)\right\rangle=\frac{\pi \hbar}{2} \delta\left(q-q^{\prime}\right) \delta\left(p-p^{\prime}\right), \quad\langle\Pi(q, p)\rangle=\langle\Pi\rangle=\frac{1}{2} .
$$

and according to definition (2.20)

$$
\begin{aligned}
(\Pi(q, p))^{2} & =D(q, p) \Pi(D(q, p))^{\dagger} D(q, p) \Pi(D(q, p))^{\dagger} \\
& =D(q, p) \Pi^{2}(D(q, p))^{\dagger}=I,
\end{aligned}
$$

where we used the property $\Pi^{2}=I$ given in (2.13).

The Wigner quasiprobability $W(q, p)$ is up to a factor $\frac{1}{\hbar \pi}$ the expectation value of the displaced parity operator $\Pi$ in the following sense

$$
W(q, p)=\frac{1}{\pi \hbar}\langle\varrho \Pi(q, p)\rangle=\frac{1}{\pi \hbar}\left\langle\varrho D(q, p) \int_{-\infty}^{+\infty} \mathrm{d} q_{0} \mid q_{0}\right\rangle\left\langle-q_{0} \mid(D(q, p))^{\dagger}\right\rangle .
$$

From this follows taking into account that $\left|q+q_{0}\right\rangle$ and $\left\langle q-q_{0}\right|$ are rightand left-hand eigenstates of $Q$

$$
\begin{aligned}
& W(q, p) \\
& =\frac{1}{\pi \hbar}\left\langle\varrho \exp \left(\mathrm{i} \frac{p Q}{\hbar}\right) \exp \left(-\mathrm{i} \frac{q P}{\hbar}\right) \int_{-\infty}^{+\infty} \mathrm{d} q_{0} \mid q_{0}\right\rangle\left\langle-q_{0} \mid \exp \left(\mathrm{i} \frac{q P}{\hbar}\right) \exp \left(-\mathrm{i} \frac{p Q}{\hbar}\right)\right\rangle \\
& =\frac{1}{\pi \hbar} \int_{-\infty}^{+\infty} \mathrm{d} q_{0}\left\langle\varrho \exp \left(\mathrm{i} \frac{p Q}{\hbar}\right) \mid q+q_{0}\right\rangle\left\langle q-q_{0} \mid \exp \left(-\mathrm{i} \frac{p Q}{\hbar}\right)\right\rangle \\
& =\frac{1}{\pi \hbar} \int_{-\infty}^{+\infty} \mathrm{d} q_{0}\left\langle\varrho \mid q+q_{0}\right\rangle\left\langle q-q_{0} \mid \varrho\right\rangle \exp \left(\mathrm{i} \frac{2 p q_{0}}{\hbar}\right),
\end{aligned}
$$

and it can now be seen that $W(q, p)$ in (2.23) is identical with the Wigner definition (2.4).

\section{Further Properties of the Wigner Quasiprobability}

We refer now further properties of the Wigner quasiprobability (e.g., [5] [6] [7] [8] [10] [19] [20] [21]). Since the density operator $\varrho$ and the displaced parity operator $\Pi(q, p)$ are Hermitean operators the Wigner quasiprobability $W(q, p)$ according to (2.23) is a real-valued function

$$
W(q, p)=(W(q, p))^{*} .
$$

However, it is not positively semi-definite and may possess regions of negativity. It is normalized if the trace of the density operator $\langle\varrho\rangle$ is normalized (integrations without writing the limits go over the whole phase space)

$$
\langle\varrho\rangle=\int \mathrm{d} q \wedge \mathrm{d} p W(q, p)=1 .
$$


The Wigner quasiprobability $W(q, p)$ contains the complete information of the density operator $\varrho$ and it can be reconstructed from the Wigner quasiprobability by the formula

$$
\varrho=2 \int \mathrm{d} q \wedge \mathrm{d} p W(q, p) \Pi(q, p) .
$$

This can be derived by inserting $\varrho$ according to (3.3) into (2.23) and using the properties (2.21) of the displaced parity operator.

The Wigner quasiprobability $W(q, p)$ is restricted by

$$
-\frac{1}{\pi \hbar} \leq W(q, p) \leq+\frac{1}{\pi \hbar} .
$$

This follows using the Cauchy-Bunyakovski-Schwarz inequality in operator form $\left\langle A^{\dagger} B\right\rangle\left\langle B^{\dagger} A\right\rangle \leq\left\langle A^{\dagger} A\right\rangle\left\langle B^{\dagger} B\right\rangle$ ( $\sqrt{\varrho}$ can be defined as positively semi-definite Hermitean operator) from

$$
\begin{aligned}
(W(q, p))^{2} & =\frac{1}{(\pi \hbar)^{2}}\langle\varrho \Pi(q, p)\rangle\langle\Pi(q, p) \varrho\rangle \\
& =\frac{1}{(\pi \hbar)^{2}}\langle\underbrace{\sqrt{\varrho} \Pi(q, p)}_{A^{\dagger}} \underbrace{\sqrt{\varrho}}_{B}\rangle\langle\underbrace{\sqrt{\varrho} \underbrace{\Pi(q, p) \sqrt{\varrho}}_{A}\rangle}_{B^{\dagger}} \\
& \leq \frac{\langle\varrho\rangle}{(\pi \hbar)^{2}}\left\langle\sqrt{\varrho}(\underbrace{\Pi(q, p))^{2}}_{=I} \sqrt{\varrho}\rangle=\frac{\langle\varrho\rangle^{2}}{(\pi \hbar)^{2}},\right.
\end{aligned}
$$

and taking into account $\langle\varrho\rangle=1$ one has proved (3.4). It can be also derived from (2.4). In comparison to the bound (3.4) a classical probability function of two real variables $(q, p)$ apart from being non-negative must not possess such a bound.

From (3.3) follows for the product of two density operators $\varrho_{1}$ and $\varrho_{2}$ to the Wigner quasiprobabilities $W_{1}(q, p)$ and $W_{2}(q, p)$ using $\Pi^{2}=I$

$$
\begin{aligned}
\varrho_{1} \varrho_{2}= & 4 \int \mathrm{d} q \wedge \mathrm{d} p \int \mathrm{d} q^{\prime} \wedge \mathrm{d} p^{\prime} W_{1}(q, p) W_{2}\left(q^{\prime}, p^{\prime}\right) \Pi(q, p) \Pi\left(q^{\prime}, p^{\prime}\right) \\
= & 4 \int \mathrm{d} q \wedge \mathrm{d} p \int \mathrm{d} q^{\prime} \wedge \mathrm{d} p^{\prime} W_{1}(q, p) W_{2}\left(q^{\prime}, p^{\prime}\right) D(q, p) \\
& \cdot \Pi D(-q,-p) D\left(q^{\prime}, p^{\prime}\right) \Pi D\left(-q^{\prime},-p^{\prime}\right) \\
= & 4 \int \mathrm{d} q \wedge \mathrm{d} p \int \mathrm{d} q^{\prime} \wedge \mathrm{d} p^{\prime} W_{1}(q, p) W_{2}\left(q^{\prime}, p^{\prime}\right) D(2 q, 2 p) D\left(-2 q^{\prime},-2 p^{\prime}\right),
\end{aligned}
$$

or if we apply the product formula (2.18) for displacement operators

$$
\begin{aligned}
\varrho_{1} \varrho_{2}= & 4 \int \mathrm{d} q \wedge \mathrm{d} p \int \mathrm{d} q^{\prime} \wedge \mathrm{d} p^{\prime} W_{1}(q, p) W_{2}\left(q^{\prime}, p^{\prime}\right) \\
& \cdot \exp \left(\mathrm{i} \frac{2\left(q p^{\prime}-p q^{\prime}\right)}{\hbar}\right) D\left(2\left(q-q^{\prime}\right), 2\left(p-p^{\prime}\right)\right) .
\end{aligned}
$$

For the trace over the product of two density operators $\varrho_{1}$ and $\varrho_{2}$ corresponding to two Wigner quasiprobabilities $W_{1}(q, p)$ and $W_{2}(q, p)$ one obtains (e.g., [2] [10] [19] [20] [21])

$$
0 \leq\left\langle\varrho_{1} \varrho_{2}\right\rangle=2 \pi \hbar \int \mathrm{d} q \wedge \mathrm{d} p W_{1}(q, p) W_{2}(q, p) \leq\left\langle\varrho_{1}\right\rangle\left\langle\varrho_{2}\right\rangle=1
$$

If the two states are mutually orthogonal that means if $\left\langle\varrho_{1} \varrho_{2}\right\rangle$ vanishes then the integral over the two Wigner functions also vanishes and vice versa, i.e. 


$$
\left\langle\varrho_{1} \varrho_{2}\right\rangle=0, \quad \Leftrightarrow \quad 0=\int \mathrm{d} q \wedge \mathrm{d} p W_{1}(q, p) W_{2}(q, p),
$$

that can be expressed as orthogonality of the corresponding Wigner quasiprobabilities. In particular, the Wigner quasiprobabilities $W_{n}(q, p)$ for the discrete orthonormalized eigenstates $\left|\psi_{n}\right\rangle,(n=0,1,2, \cdots)$ of a Hermitean operator are orthogonal to each other and in orthormalized form one finds

$$
\delta_{m, n}=\left|\left\langle\psi_{m} \mid \psi_{n}\right\rangle\right|^{2}=2 \pi \hbar \int \mathrm{d} q \wedge \mathrm{d} p W_{m}(q, p) W_{n}(q, p),\left(\left\langle\psi_{m} \mid \psi_{n}\right\rangle=\delta_{m, n}\right) .
$$

This is only possible if with exception of the ground state $\left|\psi_{0}\right\rangle$ of a Hamiltonian the Wigner quasiprobability to all other states possesses regions of negativities.

In case of $\varrho_{1}=\varrho_{2} \equiv \varrho$ one finds from (3.8)

$$
\left\langle\varrho^{2}\right\rangle=2 \pi \hbar \int \mathrm{d} q \wedge \mathrm{d} p(W(q, p))^{2} \leq 1, \quad\left\langle\varrho^{2}\right\rangle=1, \quad \Rightarrow \quad \varrho=|\psi\rangle\langle\psi|,
$$

For $\left\langle\varrho^{2}\right\rangle=1$ one has a pure state that requires the equality sign in (3.11) for the integral over the squared Wigner quasiprobability that can be used as indicator for a pure state.

\section{The Wigner Quasiprobability for a Particle in a Square Well with Infinitely High Impenetrable Walls}

As an example, we consider the stationary Schrödinger equation for a particle in a square well with width a symmetrically to the coordinate origin $q=0$ and with infinitely high impenetrable walls (e.g., [22]) (Figure 1)

$$
\left\{-\frac{\hbar^{2}}{2 \mu} \frac{\partial^{2}}{\partial q^{2}}+U(q)\right\} \psi_{n}(q)=E_{n} \psi(q) \text {. }
$$

The wave functions $\psi_{n}(q) \equiv\langle q \mid \psi\rangle$ of the eigenstates $\left|\psi_{n}\right\rangle$ to energy $E_{n}$ ( $\mu$ mass of particle)

$$
\begin{gathered}
E_{n}=\frac{\pi^{2} \hbar^{2}}{2 \mu a^{2}}(n+1)^{2} \equiv E_{0} \varepsilon_{n}, \quad E_{0} \equiv \frac{\pi^{2} \hbar^{2}}{2 \mu a^{2}}, \\
\varepsilon_{n}=(n+1)^{2}, \quad(n=0,1,2, \cdots),
\end{gathered}
$$

as it is well known, are $(\theta(x)=0,(x<0), \theta(x)=1,(x>0)$ Heaviside jump function)

$$
\begin{aligned}
\psi_{n}(q) & =\sqrt{\frac{2}{a}} \sin \left((n+1) \pi\left(\frac{1}{2}+\frac{q}{a}\right)\right) \theta\left(\frac{a}{2}-|q|\right) \\
& =(-1)^{n} \psi_{n}(-q), \quad(n=0,1,2, \cdots),
\end{aligned}
$$

In representation by the coordinate of the canonical momentum $\psi_{n}(p) \equiv\langle p \mid \psi\rangle \quad($ see $(2.5))$ they are (Figure 1)

$$
\psi_{n}(p)=\frac{1}{2} \sqrt{\frac{a}{\pi \hbar}}\left\{\mathrm{i}^{n} \frac{\sin \left(\frac{p a-(n+1) \pi \hbar}{2 \hbar}\right)}{\frac{p a-(n+1) \pi \hbar}{2 \hbar}}+(-\mathrm{i})^{n} \frac{\sin \left(\frac{p a+(n+1) \pi \hbar}{2 \hbar}\right)}{\frac{p a+(n+1) \pi \hbar}{2 \hbar}}\right\},
$$


Square well with infinitely high walls

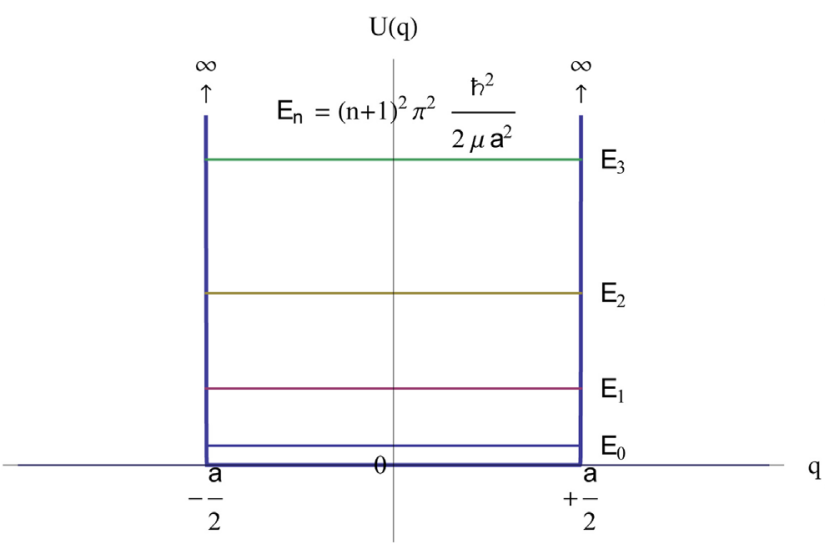

First 6 eigenfunctions in position representation

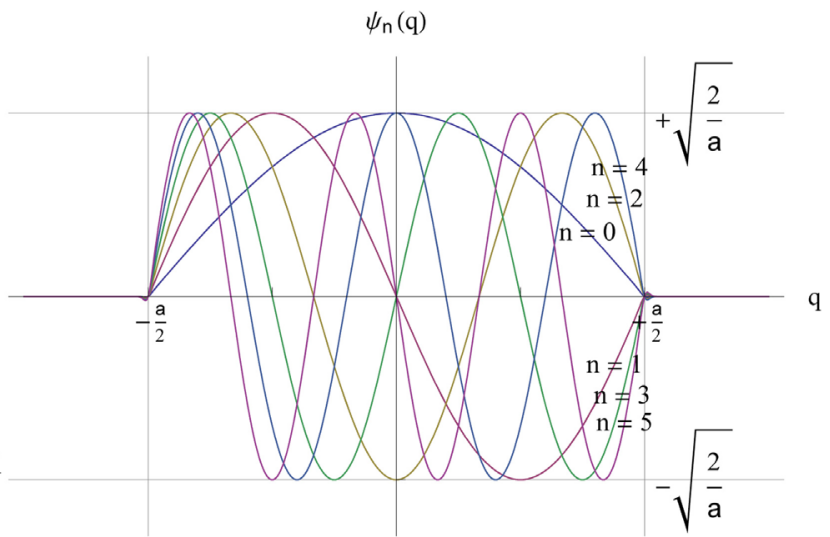

First 3 even eigenfunctions in momentum representation First 3 odd eigenfunctions in momentum representation
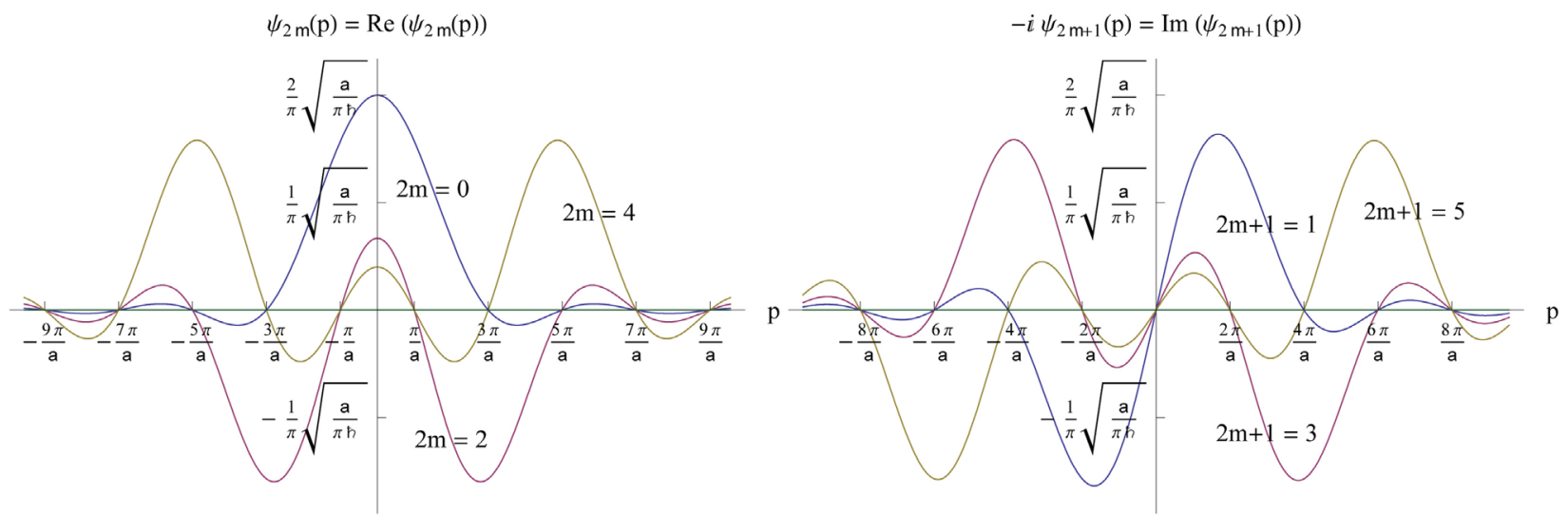

Figure 1. Square well potential with infinitely high walls and first 6 eigenfunctions in position and momentum representation.

$$
\operatorname{Im}\left(\psi_{2 m}(p)\right)=0, \quad \operatorname{Re}\left(\psi_{2 m+1}(p)\right)=0, \quad(m=0,1, \cdots) .
$$

For the Wigner quasiprobability $W(q, p)$ of an arbitrary state with density operator $\varrho$

$$
W(q, p)=\sum_{m=0}^{\infty} \sum_{n=0}^{\infty}\left\langle\psi_{m}|\varrho| \psi_{n}\right\rangle W_{n, m}(q, p)
$$

calculated from (2.4) we find

$$
\begin{aligned}
& W_{n, m}(q, p)=\frac{1}{2 \pi \hbar} \theta\left(\frac{a}{2}-|q|\right) \\
& \left\{\begin{array}{l}
\exp \left(+\mathrm{i} \frac{n-m}{2} \pi\left(1+\frac{2 q}{a}\right)\right) \frac{\sin \left(\left(\frac{p a}{\hbar}+\frac{n+m+2}{2} \pi\right)\left(1-\frac{2|q|}{a}\right)\right)}{\frac{p a}{\hbar}+\frac{n+m+2}{2} \pi} \\
+\exp \left(-\mathrm{i} \frac{n-m}{2} \pi\left(1+\frac{2 q}{a}\right)\right) \frac{\sin \left(\left(\frac{p a}{\hbar}-\frac{n+m+2}{2} \pi\right)\left(1-\frac{2|q|}{a}\right)\right)}{\frac{p a}{\hbar}-\frac{n+m+2}{2} \pi}
\end{array}\right.
\end{aligned}
$$




$$
\begin{aligned}
& -\exp \left(+\mathrm{i} \frac{n+m+2}{2} \pi\left(1+\frac{2 q}{a}\right)\right) \frac{\sin \left(\left(\frac{p a}{\hbar}+\frac{n-m}{2} \pi\right)\left(1-\frac{2|q|}{a}\right)\right)}{\frac{p a}{\hbar}+\frac{n-m}{2} \pi} \\
& \left.-\exp \left(-\mathrm{i} \frac{n+m+2}{2} \pi\left(1+\frac{2 q}{a}\right)\right) \frac{\sin \left(\left(\frac{p a}{\hbar}-\frac{n-m}{2} \pi\right)\left(1-\frac{2|q|}{a}\right)\right)}{\frac{p a}{\hbar}-\frac{n-m}{2} \pi}\right\} .
\end{aligned}
$$

For the eigenstates $\left|\psi_{n}\right\rangle$ of the Hamilton operator to this system this leads to

$$
\begin{aligned}
W_{n, n}(q, p)= & \frac{\theta\left(\frac{a}{2}-|q|\right)}{2 \pi \hbar}\left\{\frac{\sin \left(\left(\frac{p a}{\hbar}+(n+1) \pi\right)\left(1-\frac{2|q|}{a}\right)\right)}{\frac{p a}{\hbar}+(n+1) \pi}\right. \\
& +\frac{\sin \left(\left(\frac{p a}{\hbar}-(n+1) \pi\right)\left(1-\frac{2|q|}{a}\right)\right)}{\frac{p a}{\hbar}-(n+1) \pi} \\
& \left.+2(-1)^{n} \cos \left(2(n+1) \pi \frac{q}{a}\right) \frac{\sin \left(\frac{p a}{\hbar}\left(1-\frac{2|q|}{a}\right)\right)}{\frac{p a}{\hbar}}\right\} .
\end{aligned}
$$

They are illustrated in Figure 2.

We emphasize here that the calculation of expectation values from the Wigner quasiprobability is by no means in every case the simplest way. Often it is much simpler to calculate them from the wave functions in position or momentum representation. However, the principal possibility to calculate them from the Wigner quasiprobability shows the way of correspondences between classical and quantum mechanics including the transition from the last to the classical phase space by approximations.

A Hamilton system of one degree of freedom with a general quadratic energy spectrum $E_{n}=A n^{2}+B n+C$ with constants $(A, B, C)$ such as $(4.2)^{3}$ can be considered by the group $S U(1,1)$ with the basis operators $\left(K_{-}, K_{+}, K_{0}\right)$ of its Lie algebra [4]

$$
\begin{aligned}
& K_{-} \equiv \sum_{n=0}^{\infty} \sqrt{\varepsilon_{n}}\left|\psi_{n}\right\rangle\left\langle\psi_{n+1}\left|=\sum_{n=0}^{\infty}(n+1)\right| \psi_{n}\right\rangle\left\langle\psi_{n+1}\right|=\left(K_{+}\right)^{\dagger}, \\
& K_{+} \equiv \sum_{n=0}^{\infty} \sqrt{\varepsilon_{n}}\left|\psi_{n+1}\right\rangle\left\langle\psi_{n}\left|=\sum_{n=0}^{\infty}(n+1)\right| \psi_{n+1}\right\rangle\left\langle\psi_{n}\right|=\left(K_{-}\right)^{\dagger},
\end{aligned}
$$

${ }^{3}$ In a general quadratic energy spectrum $E_{n}=A n^{2}+B n+C$ with three real constants $(A, B, C)$ our abbreviation $E_{0}$ in (4.2) corresponds to the more general $E_{0} \rightarrow A$ and $\varepsilon_{n}$ to general $\varepsilon_{n} \rightarrow n^{2}+\frac{B}{A} n+\frac{C}{A}$. 

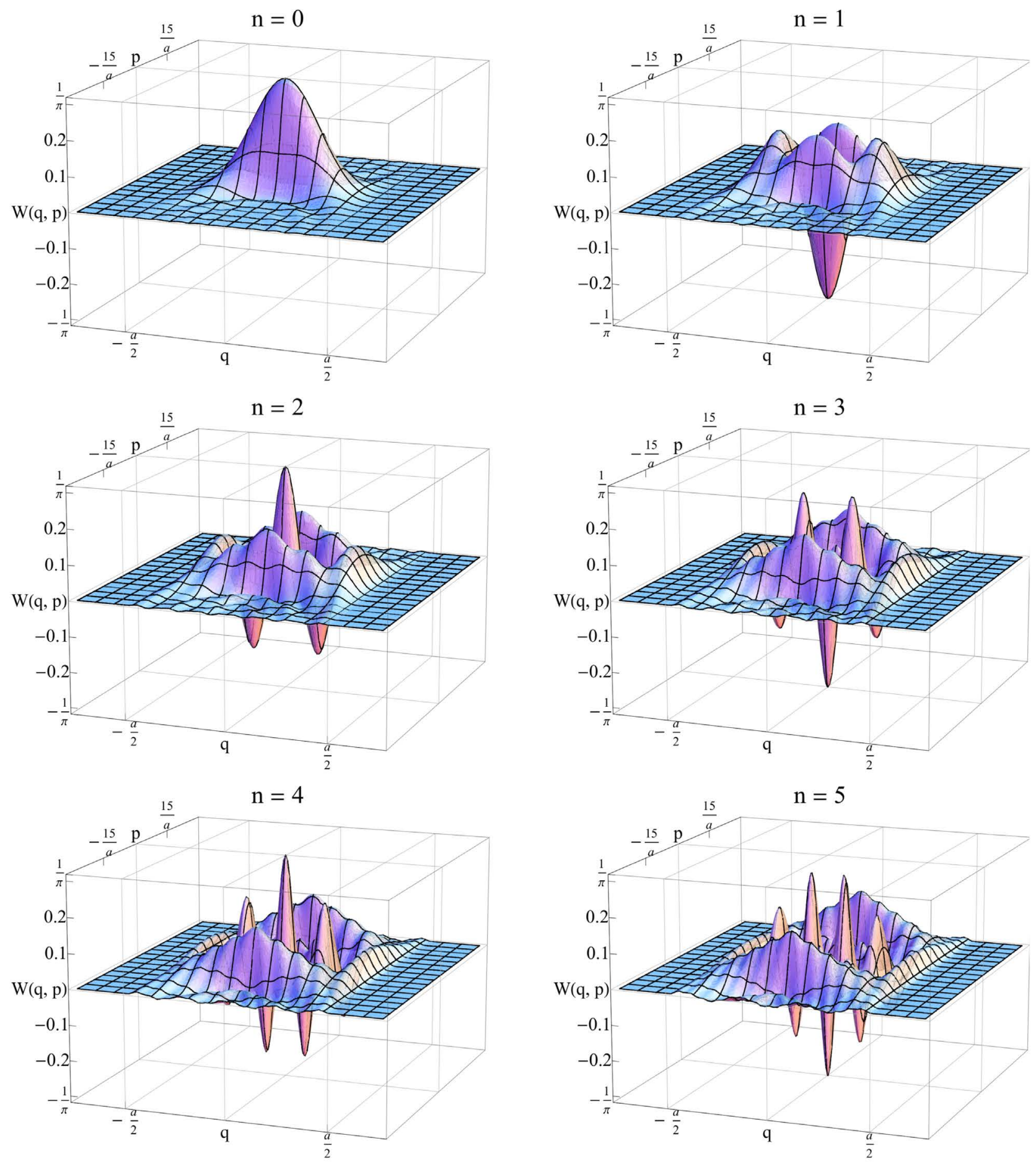

Figure 2. Wigner quasiprobabilities for first 6 eigenstates $\left|\psi_{n}\right\rangle$ of square well with infinitely high impenetrable walls $(n=0,1, \cdots, 5) . W(q, p)$ vanishes for $|q| \geq \frac{a}{2}$. We set $\hbar=1$; (made by "Mathematica 6 " in 2011).

$$
K_{0} \equiv \frac{1}{2} \sum_{n=0}^{\infty}\left(\varepsilon_{n}-\varepsilon_{n-1}\right)\left|\psi_{n}\right\rangle\left\langle\psi_{n}\left|=\sum_{n=0}^{\infty}\left(n+\frac{1}{2}\right)\right| \psi_{n}\right\rangle\left\langle\psi_{n}\right|=\left(K_{0}\right)^{\dagger},
$$

which satisfy the commutation relations 


$$
\left[K_{-}, K_{+}\right]=2 K_{0}, \quad\left[K_{0}, K_{-}\right]=-K_{-}, \quad\left[K_{0}, K_{+}\right]=+K_{+} .
$$

The Casimir operator $C$ as invariant of the considered irreducible representation is

$$
\begin{aligned}
C & \equiv\left(K_{0}\right)^{2}-\frac{1}{2}\left(K_{-} K_{+}+K_{+} K_{-}\right) \\
& =-\frac{1}{4} \sum_{n=0}^{\infty}\left|\psi_{n}\right\rangle\left\langle\psi_{n}\right|=-\frac{1}{4} I \equiv k(k-1) I, \quad \Rightarrow \quad k=\frac{1}{2} .
\end{aligned}
$$

Thus the considered system corresponds to the index $k=\frac{1}{2}$ of the irreducible representation. The completeness relation is here $\sum_{n=0}^{\infty}\left|\psi_{n}\right\rangle\left\langle\psi_{n}\right|=I$.

The Hamilton operator $H$ of the system can be represented by

$$
H=E_{0} K_{-} K_{+}=E_{0} \frac{1}{2}\left(K_{-} K_{+}+K_{+} K_{-}+I\right)=E_{0} \sum_{n=0}^{\infty}(n+1)^{2}\left|\psi_{n}\right\rangle\left\langle\psi_{n}\right| .
$$

In addition we introduce the operators $E_{-}$and $E_{+}$by

$$
E_{-} \equiv \sum_{n=0}^{\infty}\left|\psi_{n}\right\rangle\left\langle\psi_{n+1}\left|=\left(E_{+}\right)^{\dagger}, E_{+} \equiv \sum_{n=0}^{\infty}\right| \psi_{n+1}\right\rangle\left\langle\psi_{n}\left|=\left(E_{-}\right)^{\dagger},\left[E_{-}, E_{+}\right]=\right| \psi_{0}\right\rangle\left\langle\psi_{0}\right| \text {. }
$$

Their commutation relations with the operator $K_{0}$ are

$$
\left[K_{0}, E_{-}\right]=-\sum_{n=0}^{\infty}\left|\psi_{n}\right\rangle\left\langle\psi_{n+1}\left|=-E_{-}, \quad\left[K_{0}, E_{+}\right]=\sum_{n=0}^{\infty}\right| \psi_{n+1}\right\rangle\left\langle\psi_{n}\right|=+E_{+} .
$$

These relations may be considered as a possible equivalent to the quantum-mechanical commutation relations for classical action $j$ and phase $\varphi$ with the classical to quantum correspondences $\left(\mathrm{e}^{\mathrm{i} \varphi} \rightarrow K_{-}, \mathrm{e}^{-\mathrm{i} \varphi} \rightarrow K_{+}\right)$if the action operator $J$ is determined by

$$
J \equiv \hbar K_{0}=\hbar \sum_{n=0}^{\infty}\left(n+\frac{1}{2}\right)\left|\psi_{n}\right\rangle\left\langle\psi_{n}\right|
$$

Hamilton operator $H$ and action operator $J$ are here not generally proportional as it is the case for a harmonic oscillator and, therefore, is omnipresent in quantum optics. Due to

$$
\begin{gathered}
E_{-} E_{+}=\sum_{n=0}^{\infty}\left|\psi_{n}\right\rangle\left\langle\psi_{n}\right|=I, \\
E_{+} E_{-}=\sum_{n=0}^{\infty}\left|\psi_{n+1}\right\rangle\left\langle\psi_{n+1}|=I-| \psi_{0}\right\rangle\left\langle\psi_{0}\right| \neq\left(E_{-} E_{+}\right)^{\dagger}=E_{-} E_{+} .
\end{gathered}
$$

The operators $E_{-}$and $E_{+}$are not unitary operators and it is not possible to determine a Hermitean phase operator from them by transition to the Logarithms $-\mathrm{i} \log \left(E_{-}\right)$or $\mathrm{i} \log \left(E_{+}\right)$. This is related to the fact that in classical theory the transition from canonical variables $(q, p)$ to action-angle variables $(j, \varphi)$ possesses a defect as a canonical transformation for $j=0$ with undetermined $\varphi$ there.

For systems such as here a quantum-mechanical particle in a potential well or, 
more generally for example, in other potentials (e.g. Pöschl-Teller potentials [22]) the Wigner quasiprobability plays a subsidiary role for the calculation of expectation values since for mixed powers of operators $Q$ and $P$ they are mostly not of great interest. The operators $Q$ and $P$ are here not involved in a symmetrical way in the Hamiltonian and, intuitively, play an "absolute" role independently of possible canonical transformations mixing the variables.

\section{Transition to Complex Conjugate Coordinates in Quantum Optics and Coherent States}

The following well-known considerations serve at once for the further introduction of our notations. The Hamilton operator $H^{\prime}$ to a one-dimensional harmonic oscillator of frequency $\omega$ in quantum mechanics of a (charged) particle in a potential $V(q)=\frac{k}{2} q^{2},\left(k=\frac{\partial^{2} V(q, p)}{\partial q^{2}}\right.$ its second derivative $)$, and with kinetic energy $T(p)=\frac{p^{2}}{2 m}$ (m mass) can be represented by

$$
H^{\prime}=\frac{k}{2} Q^{\prime 2}+\frac{1}{2 m} P^{\prime 2}=\frac{\omega}{2}\left(m \omega Q^{\prime 2}+\frac{1}{m \omega} P^{\prime 2}\right), \quad \omega \equiv \sqrt{\frac{k}{m}}
$$

By a unitary transformation $\left(Q^{\prime}, P^{\prime}\right) \leftrightarrow(Q, P)=\left(\sqrt{m \omega} Q^{\prime}, \frac{1}{\sqrt{m \omega}} P^{\prime}\right)$ the Hamiltonian $H^{\prime}$ is transformed to

$$
H=\frac{\omega}{2}\left(Q^{2}+P^{2}\right)
$$

which is the usual form for a member (mode) of the sum of harmonic oscillators into which a bounded electromagnetic field in quantum optics can be decomposed. Canonical coordinate and momentum $(Q, P)$ are then in "symmetric way" related to the electric and magnetic field and have nothing to do with "coordinate" and moment of the mode as wave packet in free space.

We introduce now the pair of boson annihilation and creation operators $\left(a, a^{\dagger}\right)$ and corresponding complex conjugate variable $\left(\alpha, \alpha^{*}\right)$ by

$a=\frac{Q+\mathrm{i} P}{\sqrt{2 \hbar}}, a^{\dagger}=\frac{Q-\mathrm{i} P}{\sqrt{2 \hbar}}, \alpha=\frac{q+\mathrm{i} p}{\sqrt{2 \hbar}}, \alpha^{*}=\frac{q-\mathrm{i} p}{\sqrt{2 \hbar}}, \frac{\mathrm{i}}{2} \mathrm{~d} \alpha \wedge \mathrm{d} \alpha^{*}=\frac{\mathrm{d} q \wedge \mathrm{d} p}{2 \hbar}$,

with the inversion

$$
Q=\sqrt{\frac{\hbar}{2}}\left(a+a^{\dagger}\right), P=-\mathrm{i} \sqrt{\frac{\hbar}{2}}\left(a-a^{\dagger}\right), q=\sqrt{\frac{\hbar}{2}}\left(\alpha+\alpha^{*}\right), p=-\mathrm{i} \sqrt{\frac{\hbar}{2}}\left(\alpha-\alpha^{*}\right) \text {. }
$$

From this results the representation of the Wigner quasiprobability by the following substitution in (2.9)

$$
\exp \left(-Q \frac{\partial}{\partial q}-P \frac{\partial}{\partial p}\right)=\exp \left(-a \frac{\partial}{\partial \alpha}-a^{\dagger} \frac{\partial}{\partial \alpha^{*}}\right)
$$

plus the substitution of $\delta(q) \delta(p)$ by $\delta\left(\alpha, \alpha^{*}\right)$ with $\int \frac{\mathrm{i}}{2} \mathrm{~d} \alpha \wedge \mathrm{d} \alpha^{*} \delta\left(\alpha, \alpha^{*}\right) f\left(\alpha, \alpha^{*}\right)=f(0,0)$. The Hamilton operator (5.2) takes on 
the form

$$
H=\hbar \frac{\omega}{2}\left(a a^{\dagger}+a^{\dagger} a\right)=\hbar \omega\left(N+\frac{1}{2} I\right), \quad N \equiv a^{\dagger} a=N^{\dagger}, \quad\left[a, a^{\dagger}\right]=I .
$$

The transition from (5.2) (or equivalently from (5.6)) to (5.1) and inversely is a special squeezing transformation with real squeezing parameter $\zeta=\zeta^{*}$ and with the correspondence $m \omega=\sqrt{\mathrm{km}} \equiv \frac{1+\zeta}{1-\zeta}$ and, therefore, a canonical transformation.

The eigenstates $|n\rangle$ (number states) of the number operator $N$ to eigenvalues $n,(n=0,1,2, \cdots)$ are

$$
N|n\rangle=n|n\rangle,|n\rangle=\frac{a^{\dagger n}}{\sqrt{n !}}|0\rangle, a|n\rangle=\sqrt{n}|n-1\rangle, a^{\dagger}|n\rangle=\sqrt{n+1}|n+1\rangle .
$$

The vacuum state $|0\rangle$ as ground state of the Hamilton operator $H$ in (5.6) for a single mode of the electromagnetic field is in quantum optics considered as the genuine vacuum to this single mode in contrast to possible squeezed vacua but, apparently, in agreement with experiments.

Coherent states $|\alpha\rangle$ are the displaced states (or excitations) of the vacuum state $|0\rangle$ as follows

$$
\begin{aligned}
|\alpha\rangle & =D\left(\alpha, \alpha^{*}\right)|0\rangle=\exp \left(-\frac{\alpha \alpha^{*}}{2}\right) \exp (\alpha a^{\dagger} \underbrace{\exp \left(-\alpha^{*} a\right)|0\rangle}_{=|0\rangle} \\
& =\exp \left(-\frac{\alpha \alpha^{*}}{2}\right) \sum_{n=0}^{\infty} \frac{\alpha^{n}}{\sqrt{n !}}|n\rangle .
\end{aligned}
$$

The displacement operator $D\left(\alpha, \alpha^{*}\right)$ in variables $\left(\alpha, \alpha^{*}\right)$ was introduced by Glauber [5] and is defined by

$$
D\left(\alpha, \alpha^{*}\right)=\exp \left(\alpha a^{\dagger}-\alpha^{*} a\right) \equiv \exp \left(-\mathrm{i} \frac{q P-p Q}{\hbar}\right)=D(q, p) .
$$

The coherent states $|\alpha\rangle$ are the right-hand eigenstates of the operator $a$ to complex eigenvalues $\alpha$

$$
a|\alpha\rangle=\alpha|\alpha\rangle, \quad\langle\alpha| a^{\dagger}=\alpha^{*}\langle\alpha|, \quad\langle\beta \mid \alpha\rangle=\exp \left(\alpha \beta^{*}-\frac{\alpha \alpha^{*}+\beta \beta^{*}}{2}\right),
$$

and they are mutually non-orthogonal and (over-)complete. This is well known [5] [6] and, e.g., [7], and is written here for convenience. It is, however, less known that the two-dimensional Fourier transformation of coherent states provides again coherent states in the following sense

$$
\frac{1}{2 \pi} \int \frac{\mathrm{i}}{2} \mathrm{~d} \alpha \wedge \mathrm{d} \alpha^{*} \exp \left(\frac{\beta \alpha^{*}-\beta^{*} \alpha}{2}\right)|\alpha\rangle=|\beta\rangle,
$$

with specialization $\beta=0$ to the vacuum state $|\beta=0\rangle \equiv|0\rangle$ (vacuum state $|0\rangle$ possesses even parity, i.e., $\Pi|0\rangle=+|0\rangle$ )

$$
\frac{1}{2 \pi} \int \frac{\mathrm{i}}{2} \mathrm{~d} \alpha \wedge \mathrm{d} \alpha^{*}|\alpha\rangle=\frac{1}{2 \pi} \int \frac{\mathrm{i}}{2} \mathrm{~d} \alpha \wedge \mathrm{d} \alpha^{*} D\left(\alpha, \alpha^{*}\right)|0\rangle=\Pi|0\rangle=|0\rangle .
$$


Relation (5.11) is one of the possible forms to show the over-completeness of the coherent states. This can be also proved using the group integral over the displacement operators (see Appendix B)

$$
\frac{1}{2 \pi} \int \frac{\mathrm{i}}{2} \mathrm{~d} \alpha \wedge \mathrm{d} \alpha^{*} D\left(\alpha, \alpha^{*}\right)=\frac{1}{4 \pi \hbar} \int \mathrm{d} q \wedge \mathrm{d} p D(q, p)=\Pi, \quad\langle\Pi\rangle=\frac{1}{2},
$$

providing the parity operator $\Pi$ as "sum" (integral) over all weighted elements of the Heisenberg-Weyl group.

A general group relation for arbitrary operators $A$ which includes the (over-)completeness of coherent states is the following $(\langle A\rangle$ is trace of $A$ )

$$
\frac{1}{\pi} \int \frac{\mathrm{i}}{2} \mathrm{~d} \alpha \wedge \mathrm{d} \alpha^{*} D\left(\alpha, \alpha^{*}\right) A\left(D\left(\alpha, \alpha^{*}\right)\right)^{\dagger}=\langle A\rangle I .
$$

It is written in [8] in another but equivalent form (Equation (3.9) there) and is the special case for the Heisenberg-Weyl group $W(2, \mathbb{R})$ (with usual basis $(Q, P, I)$ of its Lie algebra) of a general relation for unitary irreducible representations of a group with an invariant measure and corresponds to the lemmas of Schur (properly to the first; the second lemma of Schur includes also inequivalent irreducible representations $)^{4}$. In Appendix B we give a derivation of this relation for the case of the Heisenberg-Weyl group.

A more special form is obtained if we insert in (5.14) the number-state operators $A=|n\rangle\langle m|$. With the following two equivalent definitions of the displaced number states $|\alpha, n\rangle$ (see Section 7 for more details)

$$
|\alpha, n\rangle \equiv D\left(\alpha, \alpha^{*}\right)|n\rangle=D\left(\alpha, \alpha^{*}\right) \frac{a^{\dagger n}}{\sqrt{n !}}|0\rangle=\frac{1}{\sqrt{n !}}\left(a^{\dagger}-\alpha^{*} I\right)^{n}|\alpha\rangle,
$$

and find from (5.14) the special form

$$
\frac{1}{\pi} \int \frac{\mathrm{i}}{2} \mathrm{~d} \alpha \wedge \mathrm{d} \alpha^{*}|\alpha, n\rangle\langle\alpha, m|=\langle m \mid n\rangle I=\delta_{m, n} I .
$$

For $m=n=0$ and $|\alpha, 0\rangle \equiv|\alpha\rangle$ results from this the well-known completeness relation of the coherent states

$$
\frac{1}{\pi} \int \frac{\mathrm{i}}{2} \mathrm{~d} \alpha \wedge \mathrm{d} \alpha^{*}|\alpha\rangle\langle\alpha|=I
$$

and for $m \neq 0, n=0$ the following series of special forms

$$
\frac{1}{\pi} \int \frac{\mathrm{i}}{2} \mathrm{~d} \alpha \wedge \mathrm{d} \alpha^{*}|\alpha\rangle\langle\alpha, m|=0, \quad(m \neq 0) .
$$

${ }^{4}$ For finite groups $G$ with $N$ elements $g \in G$ and dimension $n_{s}$ of the irreducible representation $D^{(s)}$ written by their matrix elements $D_{i, j}^{(s)}(q)$ this possesses the form

$$
\frac{n_{s}}{N} \sum_{g \in G} D_{i, j}^{(s)}(g) D_{k, l}^{(s)}\left(g^{-1}\right)=\frac{n_{s}}{N} \sum_{g \in G} D_{i, j}^{(s)}(g)\left(D_{l, k}^{(s)}(g)\right)^{*}=\delta_{i, l} \delta_{j, k} .
$$

If we sum over an operator $A$ in its matrix representation $A_{i, j}$ one finds

$$
\sum_{j=1}^{n_{s}} \sum_{k=1}^{n_{s}}\left(\frac{n_{s}}{N} \sum_{g \in G} D_{i, j}^{(s)}(g) A_{j, k}\left(D_{l, k}^{(s)}(g)\right)^{*}\right)=\left(\sum_{j=1}^{n_{s}} A_{j, j}\right) \delta_{i, l} \equiv\langle A\rangle \delta_{i, l}
$$

that is analogous to the form (5.14). 
This operator relation can be multiplied by arbitrary states $|\psi\rangle$ and we obtain different forms of linear dependence between the coherent states.

The parity operator $\Pi$ using the completeness of the coherent states (5.17) can be represented now by

$$
\Pi=\frac{1}{\pi} \int \frac{\mathrm{i}}{2} \mathrm{~d} \beta \wedge \mathrm{d} \beta^{*}|\beta\rangle\left\langle\beta\left|\Pi=\frac{1}{\pi} \int \frac{\mathrm{i}}{2} \mathrm{~d} \beta \wedge \mathrm{d} \beta^{*}\right| \beta\right\rangle\left\langle-\beta\left|=\sum_{n=0}^{\infty}(-1)^{n}\right| n\right\rangle\langle n| .
$$

Using the action of the displacement operators from this relation follows

$$
\begin{aligned}
\Pi\left(\alpha, \alpha^{*}\right) & \equiv D\left(\alpha, \alpha^{*}\right) \Pi\left(D\left(\alpha, \alpha^{*}\right)\right)^{\dagger} \\
& =\frac{1}{\pi} \int \frac{\mathrm{i}}{2} \mathrm{~d} \beta \wedge \mathrm{d} \beta^{*} \exp \left(\alpha \beta^{*}-\alpha^{*} \beta\right)|\alpha+\beta\rangle\langle\alpha-\beta| .
\end{aligned}
$$

where $|\alpha, j\rangle$ are displaced number states $|j\rangle$. Thus the displaced parity operator is the Fourier transformation of the operator $\frac{|\alpha+\beta\rangle\langle\alpha-\beta|}{\pi}$ with respect to variables $\left(\beta, \beta^{*}\right)$.

\section{The Wigner Quasiprobability in Complex Conjugate Coordinates and Related Quasiprobabilities of Quantum Optics}

The coherent states form the basis of the representations of the quasiprobabilities used in quantum optics. The representation of $W\left(\alpha, \alpha^{*}\right)$ in complex conjugate coordinates $\left(\alpha, \alpha^{*}\right)$ by the displaced parity operator and its representation by displaced number states (5.15) possesses the form (see Section 2, Equation (2.23) and also [23], Equation (4.1) there)

$$
W\left(\alpha, \alpha^{*}\right)=\frac{2}{\pi}\left\langle\varrho \Pi\left(\alpha, \alpha^{*}\right)\right\rangle=\frac{2}{\pi} \sum_{n=0}^{\infty}(-1)^{n}\langle\alpha, n|\varrho| \alpha, n\rangle
$$

Inserting the representation (5.20) for the displaced parity operator into this relation immediately follows

$$
W\left(\alpha, \alpha^{*}\right)=\frac{2}{\pi^{2}} \int \frac{\mathrm{i}}{2} \mathrm{~d} \beta \wedge \mathrm{d} \beta^{*} \exp \left(\alpha \beta^{*}-\alpha^{*} \beta\right)\langle\alpha-\beta|\varrho| \alpha+\beta\rangle .
$$

In its structure this is up to a constant factor a two-dimensional Fourier transformation of the matrix elements $\langle\alpha-\beta|\varrho| \alpha+\beta\rangle$ where the involved states are the coherent states ${ }^{5}$. In this sense it is widely in analogy two the one-dimensional Fourier transformation of the matrix elements $\left\langle q-q_{0}|\varrho| q+q_{0}\right\rangle$ in the definition (2.4) given by Wigner (or equivalently in (2.6)). Apart from the possibility of alternative calculations of the Wigner quasiprobability for special states the knowledge of different representation may become important in case that one gains a deeper insight in the nature of the emergence of such functions.

${ }^{5}$ We mention the identity $\exp \left(\alpha \beta^{*}-\alpha^{*} \beta\right)=\exp \left(-\mathrm{i} \frac{q p^{\prime}-p q^{\prime}}{\hbar}\right)$ in real variables by $\alpha \equiv \frac{q+\mathrm{i} p}{\sqrt{2 \hbar}}$ and $\beta \equiv \frac{q^{\prime}+\mathrm{i} p^{\prime}}{\sqrt{2 \hbar}}$. 
The reconstruction of the density operator $\varrho$ from the Wigner quasiprobability $W\left(\alpha, \alpha^{*}\right)$ can be made by the formula

$$
\varrho=2 \int \frac{\mathrm{i}}{2} \mathrm{~d} \alpha \wedge \mathrm{d} \alpha^{*} W\left(\alpha, \alpha^{*}\right) \Pi\left(\alpha, \alpha^{*}\right) .
$$

The Wigner quasiprobability in representation by the variables $\left(\alpha, \alpha^{*}\right)$ is restricted in its values by

$$
-\frac{2}{\pi} \leq W\left(\alpha, \alpha^{*}\right) \leq \frac{2}{\pi}
$$

As another possible basic definition of the (normalized) Wigner quasiprobability in complex conjugate coordinates $\left(\alpha, \alpha^{*}\right)$ follows from (2.9) using (5.5)

$$
W\left(\alpha, \alpha^{*}\right) \equiv\left\langle\varrho \exp \left(-a \frac{\partial}{\partial \alpha}-a^{\dagger} \frac{\partial}{\partial \alpha^{*}}\right)\right\rangle \delta\left(\alpha, \alpha^{*}\right),
$$

where the delta "functions" in representation by real coordinates and complex conjugate coordinates are related by $\delta(q) \delta(p)=\frac{1}{2 \hbar} \delta\left(\alpha, \alpha^{*}\right)$. Using (6.1) and taking into account that $\varrho$ is an arbitrary density operator from (6.5) follows the pure operator relation

$$
\exp \left(-a \frac{\partial}{\partial \alpha}-a^{\dagger} \frac{\partial}{\partial \alpha^{*}}\right) \delta\left(\alpha, \alpha^{*}\right)=\frac{2}{\pi} \sum_{n=0}^{\infty}(-1)^{n}|\alpha, n\rangle\langle\alpha, n| \equiv \frac{2}{\pi} \Pi\left(\alpha, \alpha^{*}\right) .
$$

It is normalized as follows by partial integration

$$
\begin{aligned}
& \int \frac{\mathrm{i}}{2} \mathrm{~d} \alpha \wedge \mathrm{d} \alpha^{*} 1 \exp \left(-a \frac{\partial}{\partial \alpha}-a^{\dagger} \frac{\partial}{\partial \alpha^{*}}\right) \delta\left(\alpha, \alpha^{*}\right) \\
& =\int \frac{\mathrm{i}}{2} \mathrm{~d} \alpha \wedge \mathrm{d} \alpha^{*} \delta\left(\alpha, \alpha^{*}\right) \underbrace{\exp \left(a \frac{\partial}{\partial \alpha}+a^{\dagger} \frac{\partial}{\partial \alpha^{*}}\right)}_{=I} 1=I .
\end{aligned}
$$

This is in agreement with

$$
\begin{aligned}
& \frac{2}{\pi} \int \frac{\mathrm{i}}{2} \mathrm{~d} \alpha \wedge \mathrm{d} \alpha^{*} \Pi\left(\alpha, \alpha^{*}\right) \\
& =2 \sum_{n=0}^{\infty}(-1)^{n} \frac{1}{\pi} \int \frac{\mathrm{i}}{2} \mathrm{~d} \alpha \wedge \mathrm{d} \alpha^{*}|\alpha, n\rangle\langle\alpha, n| \\
& =2 \lim _{\varepsilon \rightarrow+0} \sum_{n=0}^{\infty}\left(-\frac{1-\varepsilon}{1+\varepsilon}\right)^{n} I=I,
\end{aligned}
$$

where we used (7.9) but the arising geometric series is only conditionally convergent.

In anti normal ordering of the operator involved in the definition (6.5) one finds

$$
\begin{aligned}
& \exp \left(-a \frac{\partial}{\partial \alpha}-a^{\dagger} \frac{\partial}{\partial \alpha^{*}}\right) \delta\left(\alpha, \alpha^{*}\right) \\
& =\exp \left(-a \frac{\partial}{\partial \alpha}\right) \exp \left(-a^{\dagger} \frac{\partial}{\partial \alpha^{*}}\right) \exp \left(-\frac{1}{2} \frac{\partial^{2}}{\partial \alpha \partial \alpha^{*}}\right) \delta\left(\alpha, \alpha^{*}\right)
\end{aligned}
$$


Introducing into (6.9) the completeness relation (5.17) of the coherent states one obtains from (6.5)

$$
\begin{aligned}
& W\left(\alpha, \alpha^{*}\right)= \exp \left(-\frac{1}{2} \frac{\partial^{2}}{\partial \alpha \partial \alpha^{*}}\right) \frac{1}{\pi} \int \frac{\mathrm{i}}{2} \mathrm{~d} \beta \wedge \mathrm{d} \beta^{*} \\
& \cdot\left\langle\rho \exp \left(-a \frac{\partial}{\partial \alpha}\right) \mid \beta\right\rangle\left\langle\beta \mid \exp \left(-a^{\dagger} \frac{\partial}{\partial \alpha^{*}}\right)\right\rangle \delta\left(\alpha, \alpha^{*}\right) \\
&= \exp \left(-\frac{1}{2} \frac{\partial^{2}}{\partial \alpha \partial \alpha^{*}}\right) \int \frac{\mathrm{i}}{2} \mathrm{~d} \beta \wedge \mathrm{d} \beta^{*} \frac{\langle\beta|\varrho| \beta\rangle}{\pi} \underbrace{\exp \left(-\beta \frac{\partial}{\partial \alpha}-\beta^{*} \frac{\partial}{\partial \alpha^{*}}\right) \delta\left(\alpha, \alpha^{*}\right)}_{=\delta\left(\alpha-\beta, \alpha^{*}-\beta^{*}\right)} \\
&=\exp \left(-\frac{1}{2} \frac{\partial^{2}}{\partial \alpha \partial \alpha^{*}}\right) \frac{\langle\alpha|\varrho| \alpha\rangle}{\pi} \equiv \exp \left(-\frac{1}{2} \frac{\partial^{2}}{\partial \alpha \partial \alpha^{*}}\right) Q\left(\alpha, \alpha^{*}\right) .
\end{aligned}
$$

One may introduce in this way by $Q\left(\alpha, \alpha^{*}\right)$ the coherent-state quasiprobability also called Husimi-Kano quasiprobability which is connected with the Wigner quasiprobability as follows (“*” is notation for convolution of two functions)

$$
\begin{aligned}
Q\left(\alpha, \alpha^{*}\right) & \equiv \exp \left(\frac{1}{2} \frac{\partial^{2}}{\partial \alpha \partial \alpha^{*}}\right) W\left(\alpha, \alpha^{*}\right) \equiv \frac{2}{\pi} \exp \left(-2 \alpha \alpha^{*}\right) * W\left(\alpha, \alpha^{*}\right) \\
& =\frac{2}{\pi} \int \frac{\mathrm{i}}{2} \mathrm{~d} \beta \wedge \mathrm{d} \beta^{*} \exp \left(-2(\alpha-\beta)\left(\alpha^{*}-\beta^{*}\right)\right) W\left(\beta, \beta^{*}\right)=\frac{\langle\alpha|\varrho| \alpha\rangle}{\pi} .
\end{aligned}
$$

The normalization of $Q\left(\alpha, \alpha^{*}\right)$ follows immediately using partial integration

$$
\begin{aligned}
& \int \frac{\mathrm{i}}{2} \mathrm{~d} \alpha \wedge \mathrm{d} \alpha^{*} Q\left(\alpha, \alpha^{*}\right) \\
& =\int \frac{\mathrm{i}}{2} \mathrm{~d} \alpha \wedge \mathrm{d} \alpha^{*} \operatorname{lexp}\left(\frac{1}{2} \frac{\partial^{2}}{\partial \alpha \partial \alpha^{*}}\right) W\left(\alpha, \alpha^{*}\right) \\
& =\int \frac{\mathrm{i}}{2} \mathrm{~d} \alpha \wedge \mathrm{d} \alpha^{*} W\left(\alpha, \alpha^{*}\right) \underbrace{\exp \left(\frac{1}{2} \frac{\partial^{2}}{\partial \alpha \partial \alpha^{*}}\right)}_{=1} 1=1 .
\end{aligned}
$$

The coherent-state quasiprobability $Q\left(\alpha, \alpha^{*}\right)$ (correspondingly $Q(q, p)$ ) is real-valued and restricted by

$$
0 \leq Q\left(\alpha, \alpha^{*}\right) \leq \frac{1}{\pi}, \quad\left(0 \leq Q(q, p) \leq \frac{1}{2 \pi \hbar}\right),
$$

due to definition (6.11). It is easy to show that similarly to the Wigner quasiprobability a displacement of the state $\varrho_{0} \rightarrow \varrho=D\left(\beta, \beta^{*}\right) \varrho_{0}\left(D\left(\beta, \beta^{*}\right)\right)^{\dagger}$ displaces the function $Q_{0}\left(\alpha, \alpha^{*}\right)$ corresponding to $\varrho_{0}$ according to $Q_{0}\left(\alpha, \alpha^{*}\right) \rightarrow Q\left(\alpha, \alpha^{*}\right)=Q_{0}\left(\alpha-\beta, \alpha^{*}-\beta^{*}\right)$. In the process of "desmoothing" the singular zero points of $Q\left(\alpha, \alpha^{*}\right)$ form the germ cells for the formation of regions of negative values of the Wigner quasiprobability $W\left(\alpha, \alpha^{*}\right)$.

In comparison to the Wigner quasiprobability $W\left(\alpha, \alpha^{*}\right)$ the quasiprobability $Q\left(\alpha, \alpha^{*}\right)$ is smoothed by the operator $\exp \left(\frac{1}{2} \frac{\partial^{2}}{\partial \alpha \partial \alpha^{*}}\right)$ that is not connected 
with a loss of information. Inversely from given $Q\left(\alpha, \alpha^{*}\right)$ according to (6.11) one may derive the basic formula (6.11) for the Wigner quasiprobability $W\left(\alpha, \alpha^{*}\right)$ obtained from $Q\left(\alpha, \alpha^{*}\right)$. In Appendix $\mathbf{C}$ we give a derivation with interesting intermediate formulae for applications.

One may consider more general real-valued quasiprobabilities with a real parameter $r$ by the following convolution of the Wigner quasiprobability with normalized Gaussian functions

$$
F_{r}\left(\alpha, \alpha^{*}\right) \equiv \exp \left(\frac{r}{2} \frac{\partial^{2}}{\partial \alpha \partial \alpha^{*}}\right) W\left(\alpha, \alpha^{*}\right)=\frac{2}{\pi|r|} \exp \left(-\frac{2 \alpha \alpha^{*}}{r}\right) * W\left(\alpha, \alpha^{*}\right) .
$$

For $r=1$ one obtains from $W\left(\alpha, \alpha^{*}\right)$ the Husimi-Kano quasiprobability $Q\left(\alpha, \alpha^{*}\right)$ and for $r=-1$ the Glauber-Sudarshan quasiprobability $P\left(\alpha, \alpha^{*}\right)$ which may be indirectly introduced by the following formula [5] [6] (Equation (7.48)) and [7] (chap. 13) $(\mathcal{N}\{\ldots\}$ means normal ordering of content in braces)

$$
\varrho=\int \frac{\mathrm{i}}{2} \mathrm{~d} \alpha \wedge \mathrm{d} \alpha^{*} P\left(\alpha, \alpha^{*}\right)|\alpha\rangle\langle\alpha|, \quad P\left(\alpha, \alpha^{*}\right) \equiv\left\langle\varrho \mathcal{N}\left\{\delta\left(a-\alpha I, a^{\dagger}-\alpha^{*} I\right)\right\}\right\rangle,
$$

with normalization

$$
\int \frac{\mathrm{i}}{2} \mathrm{~d} \alpha \wedge \mathrm{d} \alpha^{*} P\left(\alpha, \alpha^{*}\right)=1
$$

At first place is here set the reconstruction formula of the density operator from the function $P\left(\alpha, \alpha^{*}\right)$.

The quasiprobability $P\left(\alpha, \alpha^{*}\right)$ possesses an interesting property. All classical normalized probability functions $P_{\mathrm{cl}}\left(\alpha, \alpha^{*}\right)$ which are by definition positively semi-definite but may be not bounded provide inserted into the formula for $\varrho$ in (6.15) a possible quantum-optical density operator of one mode. For coherent states $|\beta\rangle$ one finds $P\left(\alpha, \alpha^{*}\right)=\delta\left(\alpha-\beta, \alpha^{*}-\beta^{*}\right)$ and from pure states these are the only states with positively semi-definite (generalized) functions as they are also possible for classical probability functions. All other classical probability functions inserted in (6.15) provide mixed quantum-optical density operators. The corresponding Wigner quasiprobabilities for such states since they are smoothed functions of $P\left(\alpha, \alpha^{*}\right)$ according to

$$
W\left(\alpha, \alpha^{*}\right)=\exp \left(\frac{1}{2} \frac{\partial^{2}}{\partial \alpha \partial \alpha^{*}}\right) P\left(\alpha, \alpha^{*}\right)=\frac{2}{\pi} \exp \left(-2 \alpha \alpha^{*}\right) * P\left(\alpha, \alpha^{*}\right),
$$

cannot possess regions of negativity or values equal to zero but they are not identical with all non-negative such functions. For example, from the pure states they do not give the squeezed coherent states. The mentioned states with classical probability functions $P\left(\alpha, \alpha^{*}\right)=P_{\mathrm{cl}}\left(\alpha, \alpha^{*}\right)$ can be considered at once as the "most classical" quantum-optical states and a measure of nonclassicality of an arbitrary state may be defined as the nearest distance to such a state (in case of pure states the nearest distance to a coherent state [24] [25]).

The possible quantum-optical functions $P\left(\alpha, \alpha^{*}\right)$ form a greater manifold compared with the possible classical probability functions $P_{\mathrm{cl}}\left(\alpha, \alpha^{*}\right)$. For the 
transition to classical optics by the limiting procedure $\hbar \rightarrow 0$ all possible quantum-optical (usually generalized) functions $P\left(\alpha, \alpha^{*}\right)$ have to make the transition into a classically possible probability function $P_{\mathrm{cl}}\left(\alpha, \alpha^{*}\right)$ which is then not specific for its quantum-optical origin that may be one key to a deeper understanding of this function which seems to be absent now. It has to be noted that for the transition $\hbar \rightarrow 0$ one has primarily to make the transition from variables $\left(\alpha, \alpha^{*}\right)$ to real canonical variables $(q, p)$ according to (6.3). Planck's constant is involved in the denominators of $\left(\alpha, \alpha^{*}\right)$ and apart from thermodynamic formulae (mixed states) does not fully appear in representations of the quasiprobabilities by the complex variables $\left(\alpha, \alpha^{*}\right)$.

\section{Wigner Quasiprobability in Number-State Representation}

In this Section we derive the number representation of the displaced number states and formulae for the Wigner quasiprobability of arbitrary states in expansions of the number states. For symmetries in the formulae it is favorable to use the Laguerre $2 \mathrm{D}$ polynomials $\mathrm{L}_{m, n}\left(z, z^{*}\right)$ instead of the usual generalized Laguerre or Laguerre-Sonin polynomials $\mathrm{L}_{n}^{k}(u)$ for the representations. The Laguerre $2 \mathrm{D}$ polynomials $\mathrm{L}_{m, n}\left(z, z^{*}\right)$ are alternatively defined by (e.g., [11])

$$
\begin{aligned}
\mathrm{L}_{m, n}\left(z, z^{*}\right) & \equiv \exp \left(-\frac{\partial^{2}}{\partial z \partial z^{*}}\right) z^{m} z^{*_{n}} \\
& =(-1)^{m+n} \exp \left(z z^{*}\right) \frac{\partial^{m+n}}{\partial z^{* m} \partial z^{n}} \exp \left(-z z^{*}\right) \\
& =\left(z-\frac{\partial}{\partial z^{*}}\right)^{m}\left(z^{*}-\frac{\partial}{\partial z}\right)^{n} 1 \\
& =\sum_{j=0}^{\{m, n\}} \frac{m ! n !}{j !(m-j) !(n-j) !}(-1)^{j} z^{m-j} z^{* n-j} \\
& =\left(\mathrm{L}_{n, m}\left(z, z^{*}\right)\right)^{*},
\end{aligned}
$$

with the special and limiting cases

$$
\mathrm{L}_{m, 0}\left(z, z^{*}\right)=z^{m}, \quad \mathrm{~L}_{0, n}\left(z, z^{*}\right)=z^{* n}, \quad \mathrm{~L}_{m, n}(0,0)=(-1)^{n} n ! \delta_{m, n} .
$$

For the derivatives of $\mathrm{L}_{m, n}\left(z, z^{*}\right)$ from (7.1) follows

$$
\frac{\partial}{\partial z} \mathrm{~L}_{m, n}\left(z, z^{*}\right)=m \mathrm{~L}_{m-1, n}\left(z, z^{*}\right), \quad \frac{\partial}{\partial z^{*}} \mathrm{~L}_{m, n}\left(z, z^{*}\right)=n \mathrm{~L}_{m, n-1}\left(z, z^{*}\right),
$$

The more general formulae

$$
\frac{\partial^{k+l}}{\partial z^{k} \partial z^{* l}} \mathrm{~L}_{m, n}\left(z, z^{*}\right)=\frac{m ! n !}{(m-k) !(n-l) !} \mathrm{L}_{m-k, n-l}\left(z, z^{*}\right)
$$

follow immediately by repeated application of derivatives from (7.3).

The relation of the Laguerre 2D polynomials to the generalized Laguerre (or Laguerre-Sonin) polynomials $\mathrm{L}_{n}^{v}(u)$ is [11]

$$
\mathrm{L}_{m, n}\left(z, z^{*}\right)=(-1)^{n} n ! z^{m-n} \mathrm{~L}_{n}^{m-n}\left(z z^{*}\right)=(-1)^{m} m ! z^{n-m} \mathrm{~L}_{m}^{n-m}\left(z z^{*}\right),
$$

that shows that the Laguerre-Sonin polynomials may be considered as the radial 
rudiments of the Laguerre $2 \mathrm{D}$ polynomials.

We now consider the displaced number states $|\alpha, n\rangle$ defined in (5.15). By expansion of the operators as functions of $a^{\dagger}$ in Taylor series and accomplishment of their actions onto the number states it is easy to obtain the following representation of the displaced number states by the number states [11] (Equation (5.8))

$$
|\alpha, n\rangle=\exp \left(-\frac{\alpha \alpha^{*}}{2}\right) \frac{(-1)^{n}}{\sqrt{n !}} \sum_{k=0}^{\infty} \frac{1}{\sqrt{k !}} \mathrm{L}_{k, n}\left(\alpha, \alpha^{*}\right)|k\rangle .
$$

We see using (7.2) that the special cases $|0, n\rangle \equiv|n\rangle$ and $|\alpha, 0\rangle \equiv|\alpha\rangle$ are correctly obtained. From (7.6) follows for the scalar products of displaced number states

$$
\begin{aligned}
\langle\beta, m \mid \alpha, n\rangle & =\exp \left(\frac{1}{2}\left(\alpha \beta^{*}-\beta \alpha^{*}\right)\right)\left\langle m\left|D\left(\alpha-\beta, \alpha^{*}-\beta^{*}\right)\right| n\right\rangle \\
& =\exp \left(\alpha \beta^{*}-\frac{\alpha \alpha^{*}+\beta \beta^{*}}{2}\right) \frac{(-1)^{n}}{\sqrt{m ! n !}} \mathrm{L}_{m, n}\left(\alpha-\beta, \alpha^{*}-\beta^{*}\right) .
\end{aligned}
$$

Another basic relation for the displaced number states follows from (5.14) inserting there $A=|n\rangle\langle m|$ (see Appendix D)

$$
\begin{aligned}
& \frac{1}{\pi} \int \frac{\mathrm{i}}{2} \mathrm{~d} \alpha \wedge \mathrm{d} \alpha^{*}|\alpha, n\rangle\langle\alpha, m| \\
& \equiv \frac{1}{\pi} \int \frac{\mathrm{i}}{2} \mathrm{~d} \alpha \wedge \mathrm{d} \alpha^{*} D\left(\alpha, \alpha^{*}\right)|n\rangle\langle m|\left(D\left(\alpha, \alpha^{*}\right)\right)^{\dagger} \\
& =\langle n \mid m\rangle I=\delta_{m, n} I, \quad(m, n=0,1,2, \cdots) .
\end{aligned}
$$

For $m=n$ this means the (over-)completeness of the displaced number states for arbitrary $n=0,1,2, \cdots$ including the coherent states $|\alpha\rangle=|\alpha, 0\rangle$ as special case $n=0$. In (7.8) the numbers $(m, n)$ are fixed. If one fixes the displacement $\left(\alpha, \alpha^{*}\right)$ one finds immediately

$$
\sum_{n=0}^{\infty}|\alpha, n\rangle\langle\alpha, n|=D\left(\alpha, \alpha^{*}\right) \underbrace{\left(\sum_{n=0}^{\infty}|n\rangle\langle n|\right)}_{=I}\left(D\left(\alpha, \alpha^{*}\right)\right)^{\dagger}=I .
$$

This is a completeness relation of the infinite set of displaced number states $|\alpha, n\rangle,(n=0,1, \cdots)$ for arbitrary fixed $\left(\alpha, \alpha^{*}\right)$ and together with (7.8) we have for them two different systems of completeness relations.

The Wigner quasiprobability in number-state representation is

$$
\begin{aligned}
W(\alpha, \alpha) & =\frac{2}{\pi} \sum_{j=0}^{\infty}(-1)^{j}\langle\alpha, j|\varrho| \alpha, j\rangle \\
& =\frac{2}{\pi} \sum_{j=0}^{\infty}(-1)^{j} \sum_{m=0}^{\infty} \sum_{n=0}^{\infty}\langle\alpha, j \mid n\rangle\langle n|\varrho| m\rangle\langle m \mid \alpha, j\rangle \\
& =\frac{2}{\pi} \sum_{m=0}^{\infty} \sum_{n=0}^{\infty}\langle n|\varrho| m\rangle \sum_{j=0}^{\infty}(-1)^{j}\langle m \mid \alpha, j\rangle\langle\alpha, j \mid n\rangle .
\end{aligned}
$$

Using now the following formula of the form of a generating function for products of Laguerre 2D polynomials [11] (Equation (8.4)) 


$$
\begin{aligned}
& \sum_{k=0}^{\infty} \frac{(-t)^{k}}{k !} \mathrm{L}_{m, k}\left(z, z^{*}\right) \mathrm{L}_{k, n}\left(w, w^{*}\right) \\
& =\exp \left(-t w z^{*}\right)(\sqrt{t})^{m+n} \mathrm{~L}_{m, n}\left(\frac{z+t w}{\sqrt{t}}, \frac{w^{*}+t z^{*}}{\sqrt{t}}\right) \\
& =\exp \left(-t w z^{*}\right) t^{n} \mathrm{~L}_{m, n}\left(z+t w, z^{*}+\frac{w^{*}}{t}\right)
\end{aligned}
$$

in the special case $t=1,\left(w, w^{*}\right)=\left(z, z^{*}\right)$ we find for the Wigner quasiprobability from (7.10)

$$
\begin{aligned}
W\left(\alpha, \alpha^{*}\right) & =\frac{2}{\pi} \exp \left(-\alpha \alpha^{*}\right) \sum_{m=0}^{\infty} \sum_{n=0}^{\infty} \frac{\langle n|\varrho| m\rangle}{\sqrt{m ! n !}} \sum_{j=0}^{\infty} \frac{(-1)^{j}}{j !} \mathrm{L}_{m, j}\left(\alpha, \alpha^{*}\right) \mathrm{L}_{j, n}\left(\alpha, \alpha^{*}\right) \\
& =\frac{2}{\pi} \exp \left(-2 \alpha \alpha^{*}\right) \sum_{m=0}^{\infty} \sum_{n=0}^{\infty} \frac{\langle n|\varrho| m\rangle}{\sqrt{m ! n !}} \mathrm{L}_{m, n}\left(2 \alpha, 2 \alpha^{*}\right) .
\end{aligned}
$$

In representation by the canonical variables $(q, p)$ this is equivalent to (see transformations (5.3) and (5.5))

$$
W(q, p)=\frac{1}{\pi \hbar} \exp \left(-\frac{q^{2}+p^{2}}{\hbar}\right) \sum_{m=0}^{\infty} \sum_{n=0}^{\infty} \frac{\langle n|\varrho| m\rangle}{\sqrt{m ! n !}} \mathrm{L}_{m, n}\left(\sqrt{\frac{2}{\hbar}}(q+\mathrm{i} p), \sqrt{\frac{2}{\hbar}}(q-\mathrm{i} p)\right)
$$

In special case of the Wigner quasiprobabilities for the number states $\rho=|n\rangle\langle n|$ one finds from (7.12) [5]

$$
\begin{aligned}
W\left(\alpha, \alpha^{*}\right) & =\frac{2}{\pi} \exp \left(-2 \alpha \alpha^{*}\right) \frac{1}{n !} \mathrm{L}_{n, n}\left(2 \alpha, 2 \alpha^{*}\right) \\
& =\frac{2}{\pi} \exp \left(-2 \alpha \alpha^{*}\right)(-1)^{n} \mathrm{~L}_{n}\left(4 \alpha \alpha^{*}\right),
\end{aligned}
$$

and from (7.13)

$$
\begin{aligned}
W(q, p) & =\frac{1}{\pi \hbar} \exp \left(-\frac{q^{2}+p^{2}}{\hbar}\right) \frac{1}{n !} \mathrm{L}_{n, n}\left(\sqrt{\frac{2}{\hbar}}(q+\mathrm{i} p), \sqrt{\frac{2}{\hbar}}(q-\mathrm{i} p)\right) \\
& =\frac{1}{\pi \hbar} \exp \left(-\frac{q^{2}+p^{2}}{\hbar}\right)(-1)^{n} \mathrm{~L}_{n}\left(\frac{2\left(q^{2}+p^{2}\right)}{\hbar}\right) .
\end{aligned}
$$

The Wigner quasiprobability $W(q, p)$ for the first 6 number states $|n\rangle,(n=0,1, \cdots, 5)$ is illustrated in Figure 3. In principle, it is known [5] but we illustrate it for easy comparison with the eigenstates of the Hamiltonian for an impenetrable square well discussed in Section 4.

\section{Coherent Phase States with London Phase States as Their Limiting Case and Quasi-Distribution of Phase}

In this and in the following Sections we discuss coherent phase states and calculate their Wigner quasiprobability.

As phase states one defines the eigenstates of phase operators. Since the phase is only defined up to multiples of $2 \pi$ only $2 \pi$-periodically functions of the phase are uniquely defined and as classical basic functions one may choose the functions $\mathrm{e}^{\mathrm{i} n 2 \pi},(n=0, \pm 1, \pm 2, \cdots)$ which play a role in Fourier analysis. For the 


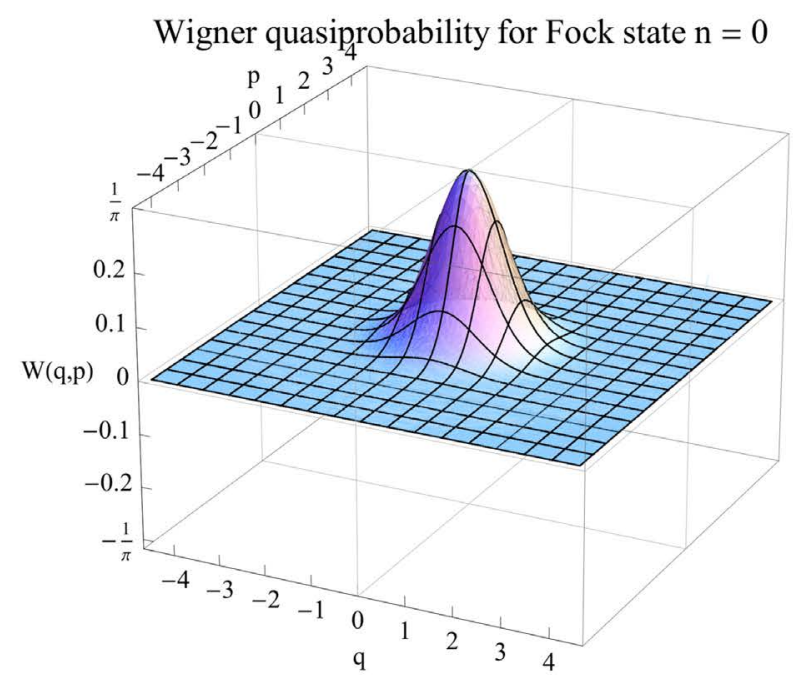

Wigner quasiprobability for Fock state $\mathrm{n}=2$

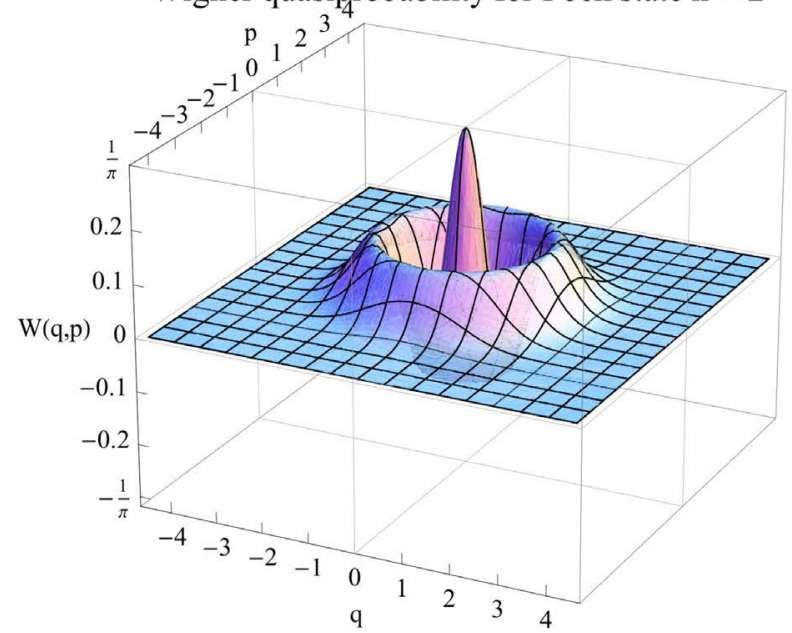

Wigner quasiprobability for Fock state $n=4$

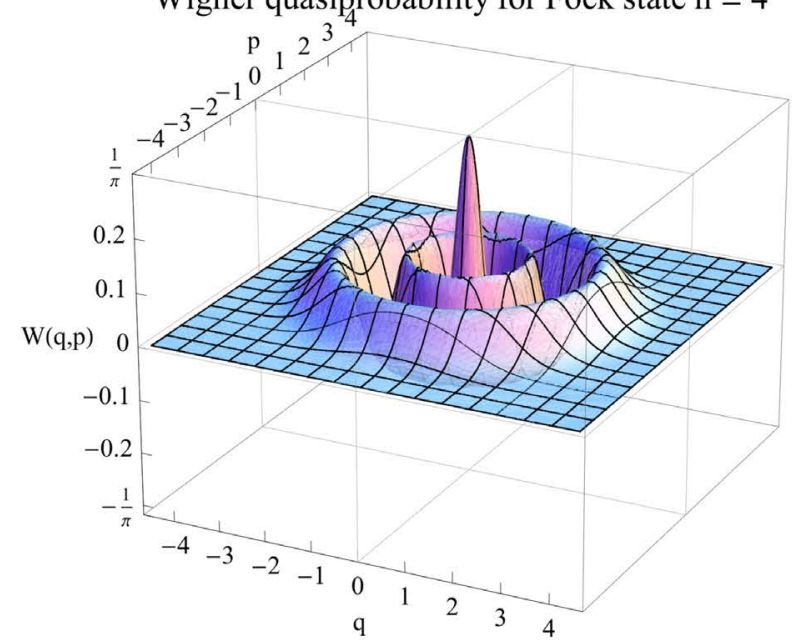

Wigner quasiprobability for Fock state $n=1$

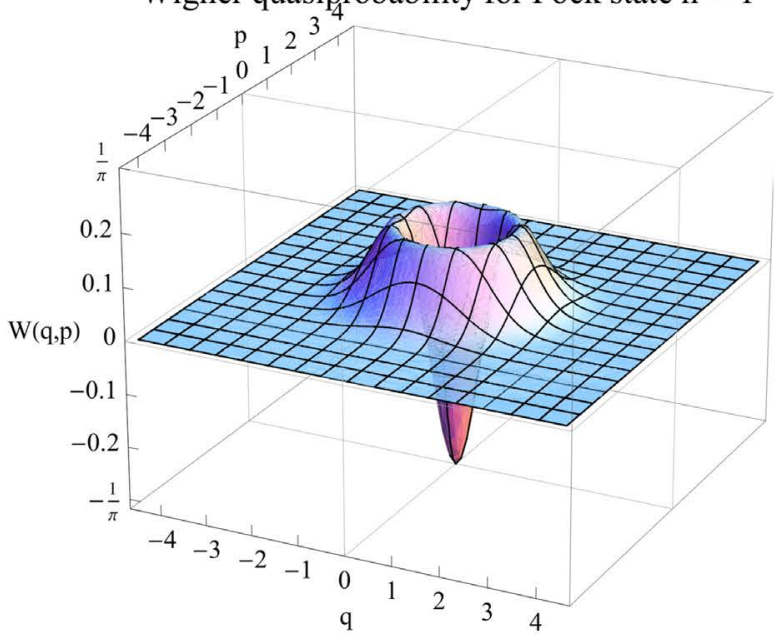

Wigner quasiprobability for Fock state $\mathrm{n}=3$

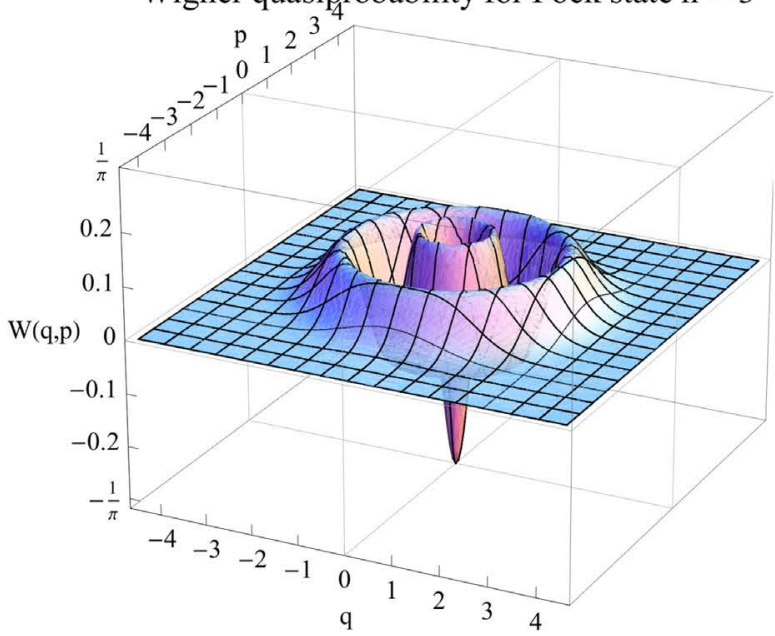

Wigner quasiprobability for Fock state $n=5$

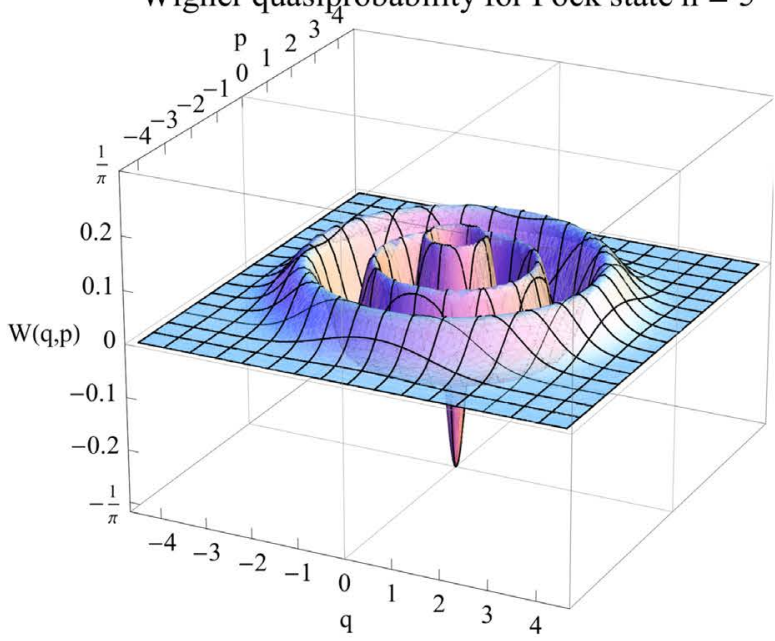

Figure 3. Wigner quasiprobability for first 6 number (Fock) states $|n\rangle$ to $n=0,1, \cdots, 5$. The maximal possible height is $\frac{1}{\pi \hbar}$ and the maximal possible depth $-\frac{1}{\pi \hbar}$. We set $\hbar=1$; (made by "Mathematica 6 " in 2011). 
quantum-mechanical equivalent operators there exist two concepts. The first is the general Weyl correspondence of symmetrically ordered operators [3] and its implementation for different categories of functions (see, e.g., [23]). The other possibility is to choose the Susskind-Glogower formalism [26] of correspondence of the operators $E_{-}$and $E_{+}$as substitution of the classical functions $\mathrm{e}^{\mathrm{i} \varphi}$ and $\mathrm{e}^{-\mathrm{i} \varphi}$ [27] [28] [29] of the phase $\varphi$ and which are

$$
E_{-}=\sum_{n=0}^{\infty}|n\rangle\left\langle n+1\left|, \quad E_{+}=\sum_{n=0}^{\infty}\right| n+1\right\rangle\left\langle n\left|, \quad\left[E_{-}, E_{+}\right]=\right| 0\right\rangle\langle 0|, \quad E_{-} E_{+}=I,
$$

where $|n\rangle$ are the number states as normalized eigenstates of the harmonic oscillator. From this follows

$$
E_{-}|n+1\rangle=|n\rangle, \quad E_{-}|0\rangle=0, \quad E_{+}|n\rangle=|n+1\rangle, \quad(n=0,1,2, \cdots) .
$$

We consider now the normalizable right-hand and left-hand eigenstates $|\varepsilon\rangle$ of the operator $E_{-}$to eigenvalues $\varepsilon$ or $\varepsilon^{*}$, respectively, according to

$$
E_{-}|\varepsilon\rangle=\varepsilon|\varepsilon\rangle, \Leftrightarrow\langle\varepsilon| E_{+}=\varepsilon^{*}\langle\varepsilon|,
$$

which are well known (e.g., [29]) and are

$$
|\varepsilon\rangle=\sqrt{1-\varepsilon \varepsilon^{*}} \sum_{n=0}^{\infty} \varepsilon^{n}|n\rangle, \quad\langle\varepsilon|=\sqrt{1-\varepsilon \varepsilon^{*}} \sum_{n=0}^{\infty}\left\langle n\left|\varepsilon^{*_{n}}, \quad\right| \varepsilon\right|<1 .
$$

They are called coherent phase states. The scalar product of two coherent phase states is

$$
\left\langle\varepsilon^{\prime} \mid \varepsilon\right\rangle=\sqrt{\left(1-\varepsilon^{\prime} \varepsilon^{\prime *}\right)\left(1-\varepsilon \varepsilon^{*}\right)} \sum_{n=0}^{\infty}\left(\varepsilon \varepsilon^{\prime^{*}}\right)^{n}=\frac{\sqrt{\left(1-\varepsilon^{\prime} \varepsilon^{\prime *}\right)\left(1-\varepsilon \varepsilon^{*}\right)}}{1-\varepsilon \varepsilon^{\prime *}}, \quad \Rightarrow \quad\langle\varepsilon \mid \varepsilon\rangle=1 .
$$

It affirms the chosen normalization factor in (8.4) and shows that the coherent phase states $|\varepsilon\rangle$ are nonorthogonal. They are (over-)complete and are already complete for arbitrary constant $|\varepsilon|$ with $0<|\varepsilon|<1$ that can be shown by contour integral in connection with the dual states [30]. That they are (over-)complete as a whole follows from $\left(\varepsilon \equiv|\varepsilon| \mathrm{e}^{\mathrm{i} \varphi}, \varepsilon^{*} \equiv|\varepsilon| \mathrm{e}^{-\mathrm{i} \varphi}\right)$

$$
\begin{aligned}
& \lim _{\delta \rightarrow 0} \frac{1}{\Gamma(\delta)} \int_{|\varepsilon| \leq 1} \frac{\mathrm{i}}{2} \mathrm{~d} \varepsilon \wedge \mathrm{d} \varepsilon^{*} \frac{|\varepsilon\rangle\langle\varepsilon|}{\left(1-|\varepsilon|^{2}\right)^{2-\delta}} \\
& =\sum_{m=0}^{\infty} \sum_{n=0}^{\infty}|m\rangle\left\langle n\left|\lim _{\delta \rightarrow 0} \frac{1}{\Gamma(\delta)} \int_{0}^{1} \mathrm{~d}\right| \varepsilon\right| \frac{|\varepsilon|^{m+n+1}}{\left(1-|\varepsilon|^{2}\right)^{1-\delta}} \int_{-\pi}^{+\pi} \mathrm{d} \varphi \mathrm{e}^{\mathrm{i}(m-n) \varphi} \\
& =2 \pi \sum_{n=0}^{\infty}|n\rangle\left\langle n\left|\lim _{\delta \rightarrow 0} \frac{1}{\Gamma(\delta)} \int_{0}^{1} \mathrm{~d}\right| \varepsilon\left|\frac{|\varepsilon|^{2 n+1}}{\left(1-|\varepsilon|^{2}\right)^{1-\delta}}=\pi \sum_{n=0}^{\infty}\right| n\right\rangle\langle n|=\pi I .
\end{aligned}
$$

In the limiting case $|\varepsilon| \rightarrow 1$ the coherent phase states $|\varepsilon\rangle$ become non-normalizable. We define

$$
|\varphi\rangle \equiv \frac{1}{\sqrt{2 \pi}} \sum_{n=0}^{\infty} \mathrm{e}^{\mathrm{i} n \varphi}|n\rangle, \quad\langle\varphi| \equiv \frac{1}{\sqrt{2 \pi}} \sum_{n=0}^{\infty} \mathrm{e}^{-\mathrm{i} n \varphi}\langle n|, \quad(0 \leq \varphi \leq 2 \pi) .
$$


These are the London phase states (after Fritz London ${ }^{6}[31]$ ). They are complete according to

$$
\int_{0}^{2 \pi} \mathrm{d} \varphi|\varphi\rangle\left\langle\varphi\left|=\sum_{m=0}^{\infty} \sum_{n=0}^{\infty}\right| m\right\rangle\left\langle n\left|\frac{1}{2 \pi} \int_{0}^{2 \pi} \mathrm{d} \varphi \mathrm{e}^{\mathrm{i}(m-n)}=\sum_{n=0}^{\infty}\right| n\right\rangle\langle n|=I .
$$

Their scalar product is

$$
\left\langle\varphi^{\prime} \mid \varphi\right\rangle=\frac{1}{2 \pi} \sum_{n=0}^{\infty} \mathrm{e}^{\mathrm{in}\left(\varphi-\varphi^{\prime}\right)}=\frac{1}{2 \pi} \frac{1}{1-\mathrm{e}^{\mathrm{i}\left(\varphi-\varphi^{\prime}\right)}} .
$$

This is a generalized function, here an analytic functional, which for $\varphi=\varphi^{\prime},(\bmod 2 \pi)$, does not give a finite value of $\langle\varphi \mid \varphi\rangle$. To make it a genuine analytic functional of variable $\varphi$ we have to determine the way to deal with the singularity at $\mathrm{e}^{\mathrm{i}\left(\varphi-\varphi^{\prime}\right)}=1$ that we will not discuss here.

The London phase states can be used to define for arbitrary (pure or mixed) states with density operator $\varrho$ a normalized $2 \pi$-periodic phase distribution $F(\varphi)$ according to $(k=0, \pm 1, \pm 2, \cdots)$

$$
F(\varphi) \equiv\langle\varphi|\varrho| \varphi\rangle \geq 0, \quad F(\varphi)=F(\varphi+2 \pi k), \quad \int_{0}^{2 \pi} \mathrm{d} \varphi F(\varphi)=1 .
$$

Since the London phase states are non-orthogonal it is not a genuine probability but only a "one-dimensional" quasiprobability (however, of other kind than the Wigner quasiprobability since it is positively semi-definite) defined over the unit circle $r=r(\varphi)=\mathrm{e}^{\mathrm{i} \varphi},(0 \leq \varphi<2 \pi)$.

For coherent phase states $|\varepsilon\rangle=|| \varepsilon\left|\mathrm{e}^{\mathrm{i} \bar{\varphi}}\right\rangle$ one finds for $F(\varphi)$

$$
F(\varphi)=\frac{1-|\varepsilon|^{2}}{2 \pi\left(1-2|\varepsilon| \cos (\varphi-\bar{\varphi})+|\varepsilon|^{2}\right)}=\frac{1-|\varepsilon|^{2}}{2 \pi\left((1-|\varepsilon|)^{2}+2 \sin ^{2}\left(\frac{\varphi-\bar{\varphi}}{2}\right)\right)} .
$$

The centered distribution for coherent phase states (i.e., for $\bar{\varphi}=0$ ) which we denote by $F_{0}(\varphi)$ possesses a very simple Fourier decomposition

$$
\begin{aligned}
F_{0}(\varphi) & \equiv \frac{1-|\varepsilon|^{2}}{2 \pi\left(1-2|\varepsilon| \cos (\varphi)+|\varepsilon|^{2}\right)}=\frac{1}{2 \pi}\left(1+2 \sum_{n=1}^{\infty}|\varepsilon|^{n} \mathrm{~T}_{n}(\cos (\varphi))\right) \\
& =\frac{1}{2 \pi}\left(1+2 \sum_{n=1}^{\infty}|\varepsilon|^{n} \cos (n \varphi)\right)=\frac{1}{2 \pi} \sum_{n=-\infty}^{+\infty}|\varepsilon|^{|n|} \mathrm{e}^{\mathrm{i} n \varphi} .
\end{aligned}
$$

The used identities are well known from the theory of Chebyshev polynomials and involve the relation $\mathrm{T}_{n}(\cos (\varphi))=\cos (n \varphi)$ and one of the generating functions for the Chebyshev polynomials of first kind $\mathrm{T}_{n}(x)$ (e.g., [32], chap. 10.11., Equations (2) and (29)). The Fourier series of the more general distribution (8.11) can be obtained from (8.12) by the substitution $\varphi \rightarrow \varphi-\bar{\varphi}$. The phase distribution (8.12) is illustrated in Figure 4 for a coherent phase state with $|\varepsilon|=0.98 \quad$ (and $\bar{\varphi}=0$ ).

The Fourier amplitudes $|\varepsilon|^{|n|}$ in (8.12) are strictly decreasing for $|\varepsilon|<1$ from $n=0$ on to both sides of $n$. In the limiting case $|\varepsilon| \rightarrow 1$ one derives or affirms from (8.12) the known relation of the theory of generalized functions

${ }^{6}$ The elder of the two brothers Fritz and Heinz London, both physicists. 
Phase distribution of coherent phase state, $|\varepsilon|=0.98$

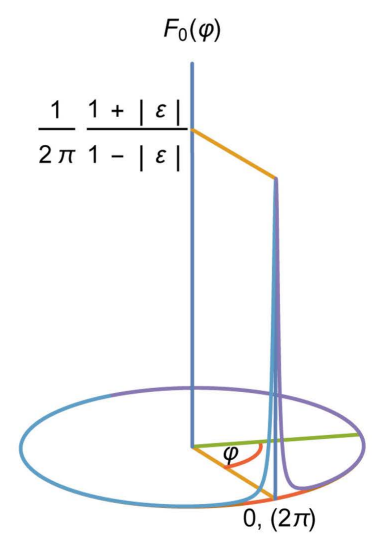

Phase distribution of coherent phase state unfolded, $|\varepsilon|=0.98$

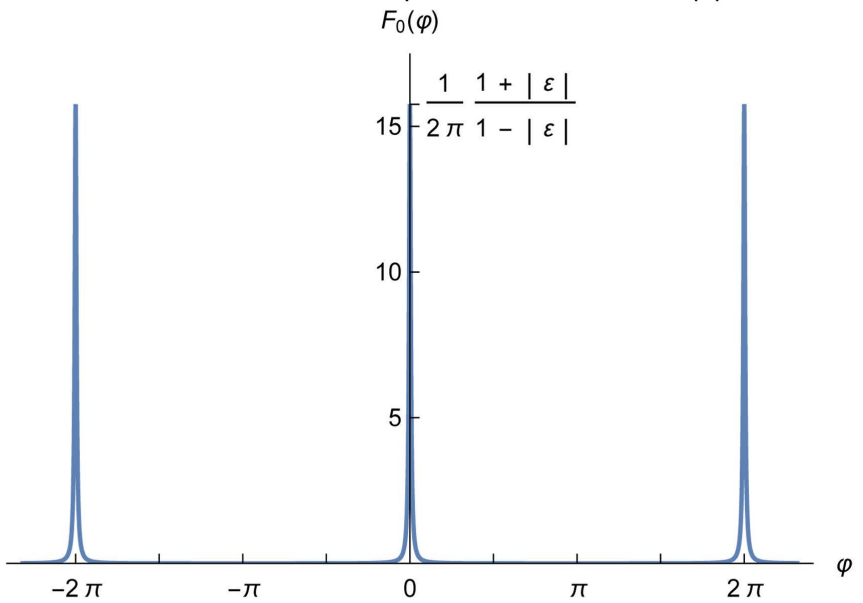

Figure 4. Phase distribution $F_{0}(\varphi)$ for a phase state $|\varepsilon=| \varepsilon\left|\mathrm{e}^{\mathrm{i} \bar{\varphi}}\right\rangle$ with $|\varepsilon|=0.98$ and $\bar{\varphi}=0$ folded and unfolded. The mean value of the number operator $N$ is $\bar{N}=24.2555$ from which follows $\frac{1}{2 \bar{N}}=0.0206461$; (“Mathematica 10" in 2018).

(e.g., Vladimirov [33], chap. 2, \$9, Equation (35))

$$
\frac{1}{2 \pi} \sum_{n=-\infty}^{+\infty} \mathrm{e}^{\mathrm{i} n \varphi}=\sum_{m=-\infty}^{+\infty} \delta(\varphi-2 \pi m)
$$

which is also an example for the Poisson transformation of a sum from ordinary to reciprocal lattice.

\section{Coherent Phase States as $S U(1,1)$ Coherent States}

Up to now we developed the elementary theory of coherent phase states $|\varepsilon\rangle$. Less elementary is that the coherent phase states are $S U(1,1)$ coherent states [12] [13] [14] [34]. The definition (8.4) of coherent phase states can be also written

$$
|\varepsilon\rangle=\sqrt{1-\varepsilon \varepsilon^{*}} \exp \left(\varepsilon \sqrt{N} a^{\dagger}\right)|0\rangle=\sqrt{1-\varepsilon \varepsilon^{*}} \sum_{n=0}^{\infty} \frac{\varepsilon^{n}}{n !}\left(\sqrt{N} a^{\dagger}\right)^{n}|0\rangle .
$$

The operators

$$
\begin{gathered}
K_{-} \equiv E_{-} N=a \sqrt{N}=\sqrt{N+I} a, \\
K_{+} \equiv N E_{+}=\sqrt{N} a^{\dagger}=a^{\dagger} \sqrt{N+I}, \\
K_{0} \equiv N+\frac{1}{2} I, \quad\left(N \equiv a^{\dagger} a\right),
\end{gathered}
$$

form a realization of the Lie algebra $S U(1,1)$ with the commutation relations (see also (4.9))

$$
\left[K_{-}, K_{+}\right]=2 K_{0}, \quad\left[K_{0}, K_{-}\right]=-K_{-},\left[K_{0}, K_{+}\right]=+K_{+},
$$

and with the Casimir operator (confer also (4.9) with the same value $k$ )

$$
C \equiv\left(K_{0}\right)^{2}-\frac{1}{2}\left(K_{-} K_{+}+K_{+} K_{-}\right)=-\frac{1}{4} I \equiv k(k-1) I, \quad \Rightarrow \quad k=\frac{1}{2} \text {. }
$$


Interestingly, the index $k=\frac{1}{2}$ of the unitary irreducible representation of $S U(1,1)$ is the same as for the system of a particle in a square well with infinitely high impenetrable walls (see Section 4).

The generation of the coherent phase states $|\varepsilon\rangle$ from the vacuum state $|0\rangle$ by a unitary operator can now be written

$$
|\varepsilon\rangle=\exp \left(\frac{\operatorname{Arth}\left(\sqrt{\varepsilon \varepsilon^{*}}\right)}{\sqrt{\varepsilon \varepsilon^{*}}}\left(\varepsilon K_{+}-\varepsilon^{*} K_{-}\right)\right)|0\rangle=\sqrt{1-\varepsilon \varepsilon^{*}} \exp \left(\varepsilon K_{+}\right)|0\rangle
$$

and (9.1) follows from the disentanglement of this unitary operator. The first relation on the right-hand side is the unitary approach that means the application of a unitary operator to the vacuum state which guarantees the normalization $\langle\varepsilon \mid \varepsilon\rangle=1$. The second relation on the right-hand side is that what we call the non-unitary approach meaning the application of the non-unitary operator $\exp \left(\varepsilon K_{+}\right)$to the vacuum state. The normalization factor $\sqrt{1-\varepsilon \varepsilon^{*}}$ can be obtained in this case by finding disentanglement relations for general group operations into products of simpler group elements. All formulae for the $S U(1,1)$ group given in Appendix A of [35] among them the disentanglement relations are applicable to the present case.

\section{Wave Functions and Further Characteristics of Coherent Phase States}

We begin with the Bargmann (-Segal) representation of states (e.g., [8]) which is the scalar product of the considered state with analytic but non-normalized coherent states $\| \alpha\rangle \equiv \exp \left(\frac{\alpha \alpha^{*}}{2}\right)|\alpha\rangle$ concerning complex variable $\alpha$ and which leads in our case of coherent phase states $|\varepsilon\rangle$ to

$$
\langle\varepsilon|| \alpha\rangle=\sqrt{1-\varepsilon \varepsilon^{*}} \sum_{n=0}^{\infty} \frac{\left(\varepsilon^{*} \alpha\right)^{n}}{\sqrt{n !}} \equiv \sqrt{1-\varepsilon \varepsilon^{*}} f\left(\varepsilon^{*} \alpha\right), \quad\langle\alpha \| \varepsilon\rangle=\langle\varepsilon \| \alpha\rangle^{*},
$$

where we introduced the very unorthodox entire function $f(z)$ of the complex variable $z$ defined by

$$
f(z) \equiv \sum_{n=0}^{\infty} \frac{z^{n}}{\sqrt{n !}}, \quad f\left(z^{*}\right)=(f(z))^{*} .
$$

This function which is difficult to deal with plays a role, practically, in all representations of the coherent phase states $|\varepsilon\rangle[36][37]^{7}$. Although the Taylor series (10.2) represents an entire function which, therefore, converges in the

\footnotetext{
${ }^{7}$ Besides a lecture about Hermite and Laguerre 2D polynomials at a Conference in Patras (Greece) I presented there in the Section "Open Problems" a short remark about the very unorthodox entire function (10.2) and the difficulties of its treatment and it was admitted and desired to publish from this one page [37]. Much later, I got an email from Skorokhodov (see Appendix D) with some information, in particular, about the zeros of this function (35 pairs with high precision). It is a pity that with my present computer I was no more able up to now to open again the appended file.
} 
whole complex plane this convergence is "very slow" and direct computer calculations from the series for high values of $|z|$ (say, for about $|z| \geq 10$ ) are very difficult to make. About some progress with this function we give a few information in Appendix D.

From the Bargmann representation (10.1) of the coherent phase states one obtains immediately the Husimi-Kano quasiprobability $Q\left(\alpha, \alpha^{*}\right)$ according to

$$
\begin{aligned}
& Q\left(\alpha, \alpha^{*}\right) \equiv \frac{\langle\alpha \mid \varepsilon\rangle\langle\varepsilon \mid \alpha\rangle}{\pi} \\
& =\frac{1-\varepsilon \varepsilon^{*}}{\pi} \exp \left(-\alpha \alpha^{*}\right) f\left(\varepsilon^{*} \alpha\right) f\left(\varepsilon \alpha^{*}\right) .
\end{aligned}
$$

From the normalization of $Q\left(\alpha, \alpha^{*}\right)$ according to (6.12) follows by the substitutions $z=\varepsilon^{*} \alpha, z^{*}=\varepsilon \alpha^{*}$

$$
\int \frac{\mathrm{i}}{2} \mathrm{~d} z \wedge \mathrm{d} z^{*} \exp \left(-\frac{z z^{*}}{\varepsilon \varepsilon^{*}}\right) f(z) f\left(z^{*}\right)=\frac{\pi|\varepsilon|^{2}}{1-|\varepsilon|^{2}}, \quad|\varepsilon|<1 .
$$

This means that $f(z) f\left(z^{*}\right)$ increases for real $z=x \rightarrow+\infty$ exponentially not faster than $\exp \left((1+\delta) x^{2}\right)$ with arbitrary small $\delta>0$. The order of the entire function is equal to $\frac{1}{2}$ (see [38] and Appendix D).

For the wave functions $\langle q \mid \varepsilon\rangle$ one finds

$$
\begin{aligned}
\langle q \mid \varepsilon\rangle & =\frac{\sqrt{1-\varepsilon \varepsilon^{*}}}{(\pi \hbar)^{\frac{1}{4}}} \exp \left(-\frac{q^{2}}{2 \hbar}\right) \sum_{n=0}^{\infty} \frac{1}{\sqrt{n !}}\left(\frac{\varepsilon}{\sqrt{2}}\right)^{n} \mathrm{H}_{n}\left(\frac{q}{\sqrt{\hbar}}\right) \\
& =\frac{\sqrt{1-\varepsilon \varepsilon^{*}}}{(\pi \hbar)^{\frac{1}{4}}} \exp \left(-\frac{q^{2}}{2 \hbar}\right) \exp \left(-\frac{\hbar}{4} \frac{\partial^{2}}{\partial q^{2}}\right) \sum_{n=0}^{\infty} \frac{1}{\sqrt{n !}}\left(\sqrt{\frac{2}{\hbar}} \varepsilon q\right)^{n},
\end{aligned}
$$

where we applied the generation of the Hermite polynomials $\mathrm{H}_{n}(z)$ from the monomials $z^{n}$ according to

$$
\mathrm{H}_{n}\left(\frac{z}{c}\right)=\exp \left(-\frac{c^{2}}{4} \frac{\partial^{2}}{\partial z^{2}}\right)\left(\frac{2 z}{c}\right)^{n},
$$

with a parameter $c$. With the function $f(z)$ defined in (10.2) this and analogously $\langle p \mid \varepsilon\rangle$ can be written

$$
\begin{array}{r}
\langle q \mid \varepsilon\rangle=\frac{\sqrt{1-\varepsilon \varepsilon^{*}}}{(\pi \hbar)^{\frac{1}{4}}} \exp \left(-\frac{q^{2}}{2 \hbar}\right) \exp \left(-\frac{\hbar}{4} \frac{\partial^{2}}{\partial q^{2}}\right) f\left(\sqrt{\left.\frac{2}{\hbar} \varepsilon q\right),}\right. \\
\langle p \mid \varepsilon\rangle=\frac{\sqrt{1-\varepsilon \varepsilon^{*}}}{(\pi \hbar)^{\frac{1}{4}}} \exp \left(-\frac{p^{2}}{2 \hbar}\right) \exp \left(-\frac{\hbar}{4} \frac{\partial^{2}}{\partial p^{2}}\right) f\left(-\mathrm{i} \sqrt{\left.\frac{2}{\hbar} \varepsilon p\right) .}\right.
\end{array}
$$

The operator $\exp \left(\frac{a^{2}}{4} \frac{\partial^{2}}{\partial x^{2}}\right)$ with $a^{2}>0$ applied to a function $f(x)$ makes a smoothing of this function and the operator $\exp \left(-\frac{a^{2}}{4} \frac{\partial^{2}}{\partial x^{2}}\right)$ with 
$a^{2}>0$, correspondingly, the opposite of this ("desmoothing").

\section{Calculation of the Wigner Quasiprobability of a Coherent Phase State}

For the calculation of the Wigner quasiprobability $W\left(\alpha, \alpha^{*}\right)$ we have different possibilities. The first is that we calculate it from the general relation to the $\mathrm{Hu}-$ simi-Kano quasiprobability $Q\left(\alpha, \alpha^{*}\right)$ and using the special form (10.3) of the last (see Appendix C)

$$
\begin{aligned}
& W\left(\alpha, \alpha^{*}\right)=\exp \left(-\frac{1}{2} \frac{\partial^{2}}{\partial \alpha \partial \alpha^{*}}\right) Q\left(\alpha, \alpha^{*}\right) \\
& =\frac{1-\varepsilon \varepsilon^{*}}{\pi} \exp \left(-\frac{1}{2} \frac{\partial^{2}}{\partial \alpha \partial \alpha^{*}}\right) \exp \left(-\alpha \alpha^{*}\right) f\left(\varepsilon^{*} \alpha\right) f\left(\varepsilon \alpha^{*}\right) \\
& =\frac{1-\varepsilon \varepsilon^{*}}{\pi} \exp \left(-\alpha \alpha^{*}\right) \exp \left\{-\frac{1}{2}\left(\frac{\partial}{\partial \alpha}-\alpha^{*}\right)\left(\frac{\partial}{\partial \alpha^{*}}-\alpha\right)\right\} f\left(\varepsilon^{*} \alpha\right) f\left(\varepsilon \alpha^{*}\right) \\
& =\frac{1-\varepsilon \varepsilon^{*}}{\pi} \exp \left\{-\left(\alpha-\frac{1}{2} \frac{\partial}{\partial \alpha^{*}}\right)\left(\alpha^{*}-\frac{1}{2} \frac{\partial}{\partial \alpha}\right)\right\} \exp \left(-\frac{1}{2} \frac{\partial^{2}}{\partial \alpha \partial \alpha^{*}}\right) f\left(\varepsilon^{*} \alpha\right) f\left(\varepsilon \alpha^{*}\right) .
\end{aligned}
$$

This is in number representation equivalent to the formula (see (7.12) and (7.13))

$$
W\left(\alpha, \alpha^{*}\right)=\frac{2}{\pi} \exp \left(-2 \alpha \alpha^{*}\right) \sum_{m=0}^{\infty} \sum_{n=0}^{\infty} \frac{\langle n \mid \varepsilon\rangle\langle\varepsilon \mid m\rangle}{\sqrt{m ! n !}} \mathrm{L}_{m, n}\left(2 \alpha, 2 \alpha^{*}\right),
$$

where $\langle n \mid \varepsilon\rangle$ and $\langle\varepsilon \mid m\rangle$ can be taken from (8.4) and which we used for the calculations. To get $W(q, p)$ we have to transform it by relations (5.3). A second possibility is the calculation directly from the wave function $\varepsilon(q) \equiv\langle q \mid \varepsilon\rangle$ or from $\varepsilon(p) \equiv\langle p \mid \varepsilon\rangle$ via definitions (2.4) or (2.6) with density operator $\rho=|\varepsilon\rangle\langle\varepsilon|$. A difficulty in the calculation of the Wigner quasiprobability for coherent phase states $|\varepsilon\rangle$ is the slow convergence of the involved series and the presence of the unorthodox entire function $f(z)$ in the formulae. The convergence is caused only by the powers $|\varepsilon|^{n}$ which for $1-|\varepsilon| \ll 1$ is very slow.

In Figure 5 we illustrate the calculation of the Wigner quasiprobability for a coherent phase state for real $\varepsilon=0.98$, i.e. for $\bar{\varphi}=0$, made by formula (C.11) using their number representation (8.4) with "Mathematica 6" in $2011^{8}$.

The maximal value of the Wigner quasiprobability occurs under increasing parameter $|\varepsilon|<1$ for increasing values $\sqrt{q^{2}+p^{2}}=\sqrt{2 \hbar}|\alpha|$ and does not reach

\footnotetext{
${ }^{8}$ After different trials we found that $|\varepsilon|=0.98$ was for us the maximally acceptable value concerning the calculation time. Trials were also made to suppress the visibility of the oscillations at the borders due to truncation of the involved series. We needed approximately 40 hours calculation time for one such picture (we did not apply any function of exponential type for acceleration of the convergence). In crass opposition to this was the pure calculation time for the given squeezed coherent state with some similarity to the Wigner quasiprobability of the coherent phase state which after programming according to the known formula was approximately of the order of a very few minutes.
} 

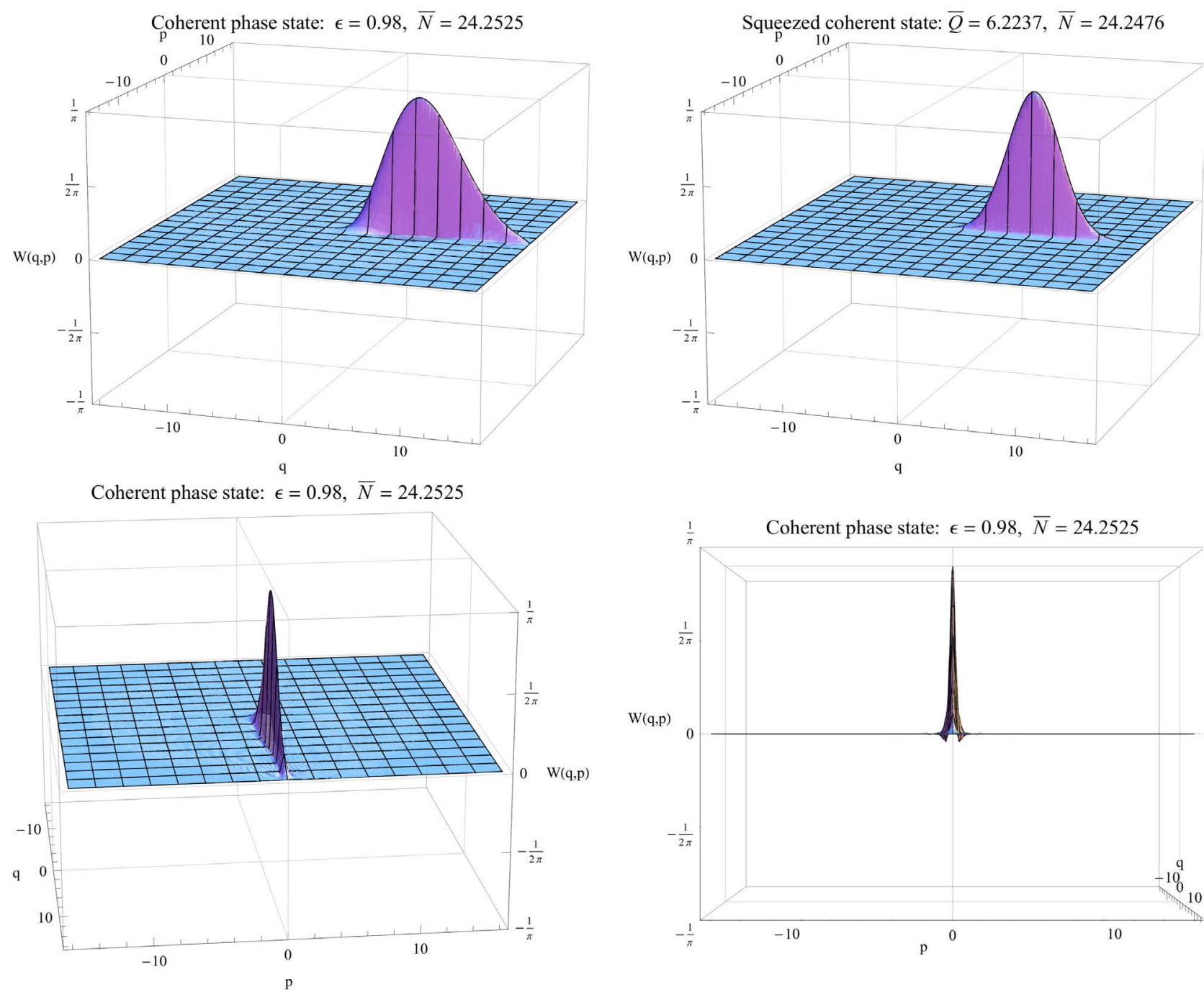

Figure 5. Wigner quasiprobability for a coherent phase state compared with a squeezed coherent state in units $\hbar$. The parameters of the coherent phase states are: $\varepsilon=0.98, \bar{N}=24.2525, \bar{Q}=6.22366, \bar{P}=0, \overline{(\Delta Q)^{2}}=10.7379, \overline{(\Delta P)^{2}}=0.033236$ and therefore $\sqrt{(\Delta Q)^{2} \cdot \overline{(\Delta P)^{2}}}=0.597398$. The parameters of the squeezed coherent state are: $\bar{N}=24.2476, \bar{Q}=6.22366$, $\bar{P}=0, \overline{(\Delta Q)^{2}}=10.7379, \overline{(\Delta P)^{2}}=\frac{1}{4 \cdot \overline{(\Delta Q)^{2}}}=0.023282$ and therefore $\sqrt{(\Delta Q)^{2} \cdot \overline{(\Delta P)^{2}}}=0.5$. We set $\hbar=1$. The partial picture in second position of second line which is a front sight onto the Wigner quasiprobability shows weakly its negativities. A good graphical representation of the small negativities is only possible by showing a small part of the Wigner quasiprobability with distortion of the natural scales. It shows that the greatest negativities lie in the neighborhood of small values of $q$ corresponding to the zeros of the non-orthodox function $f(z)$; (made by "Mathematica 6" in 2011).

the maximally possible value $\frac{1}{\pi}$ for Wigner quasiprobabilities, in contrast to that for squeezed coherent states, which apart from their displacement from the coordinate origin in comparison the squeezed vacuum states are eigenstates of the parity operator to parity +1 . The Wigner quasiprobability for coherent phase states possesses also regions of negativity. They appear in the neighborhood of the (singular) zeros of the Husimi-Kano quasiprobability $Q(q, p)$ since the last 
is some averaging of the Wigner quasiprobability and this is only possible if the Wigner quasiprobability possesses regions of negativity in its neighborhood. According to (10.3) the singular zeros of $Q(q, p)$ (or $Q\left(\alpha, \alpha^{*}\right)$ ) are determined by the zeros of the unorthodox function $f(z)$ (see also Appendix D). That the Wigner quasiprobability for coherent phase states should possess regions of negativity is also clear from the product (Weierstrass) representation of analytic functions because such functions without zeros are functions of the exponential type and the squeezed coherent states are already the most general set of normalizable functions with wave functions and Wigner quasiprobabilities of Gaussian type.

Let us make a few remarks about the article [15]. According to its title, in this article the Wigner function of a phase state is calculated and some related functions are represented graphically. As phase state the authors use the London phase states $|\varphi\rangle$ given here in (8.7) which are non-normalizable (see (8.9)). They declare $\rho=|\varphi\rangle\langle\varphi|$ as the density operator but it is $\langle\varphi \mid \varphi\rangle=\infty$ and the obtained "Wigner function" is also non-normalizable and, therefore, is not a genuine Wigner quasiprobability. Expectation values cannot be calculated with such a function. One may think that the calculated function plays, at least, an auxiliary role for the calculation of interesting properties of normalizable phase states $|\varepsilon\rangle=\left|\mathrm{e}^{\mathrm{i} \bar{\varphi}}\right| \varepsilon|\rangle$ for $|\varepsilon|<1$ defined in (8.4) from which the London phase states (8.7) follow as their limiting case $|\varepsilon|=1$ without the normalization factor $\sqrt{1-|\varepsilon|^{2}}$. However, we did not find a relation where they may play an auxiliary role. The authors [15] made their London phase states finite by truncating the sum over $n$ in (8.7) with certain $n=n_{\max }$ but then the scalar product of these changed states is $\frac{n_{\max }}{2 \pi}$ and is not normalized to the value equal to 1 and the results for the Wigner function are badly defined. In case of our normalizable coherent phase states $|\varepsilon\rangle$ the truncation is made "in natural way" by the powers $|\varepsilon|^{n},(|\varepsilon|<1)$ for $n \rightarrow \infty$ which is a very slowly convergent procedure and which caused our extremely long calculation times.

We have to underline the following. To get use from the calculated functions in [15] (or its generalization for $\varphi \neq \varphi^{\prime}$ ) as an auxiliary quantity for calculations, for example, of expectation values it has to be given, at least, in general analytic form. However, due to the involved unorthodox entire function $f(z)$ this is very difficult to achieve. This also concerns our calculations of a genuine example of the Wigner quasiprobability for coherent phase states $|\varepsilon\rangle$ in Figure 5. This may only serve as a certain illustration but for the calculation of expectation values from the Wigner quasiprobability $W(q, p)$ this is inappropriate. Some expectation values for the coherent phase states $|\varepsilon\rangle$ can be effectively calculated in other way that we demonstrate in the next Sections.

\section{Expectation Values of Functions of the Number Operator $N$ for Coherent Phase States}

The calculation of expectation values via the Wigner quasiprobability is the most 
illustrative one concerning the analogy to classical phase space but it is not in every case the simplest one and in case of coherent phase states the difficulties are almost not to overcome. Therefore, we discuss here and in the next Section some other possibilities of calculation of expectation values for coherent phase states $|\varepsilon\rangle$.

The expectation values $\overline{N^{k}}$ of powers of the number operator $N$ are

$$
\begin{aligned}
\overline{N^{0}}=1, & \\
\overline{N^{k}} & =\left(1-|\varepsilon|^{2}\right) \sum_{n=0}^{\infty}(n+1)^{k}|\varepsilon|^{2(n+1)} \\
& =\frac{|\varepsilon|^{2}}{\left(1-|\varepsilon|^{2}\right)^{k}} \sum_{l=0}^{k} E_{1}(k, l)|\varepsilon|^{2 l}, \quad(k=1,2, \cdots),
\end{aligned}
$$

where $E_{1}(k, l)$ denotes the Eulerian numbers [17] [18] and also [16] with some of the first given in Table 1.

In particular

$$
\begin{gathered}
\bar{N}=\frac{|\varepsilon|^{2}}{1-|\varepsilon|^{2}}, \Leftrightarrow|\varepsilon|=\sqrt{\frac{\bar{N}}{1+\bar{N}}} \\
\overline{N^{2}}=\frac{|\varepsilon|^{2}\left(1+|\varepsilon|^{2}\right)}{\left(1-|\varepsilon|^{2}\right)^{2}}, \overline{(\Delta N)^{2}} \equiv \overline{N^{2}}-\bar{N}^{2}=\frac{|\varepsilon|^{2}}{\left(1-|\varepsilon|^{2}\right)^{2}} \\
\overline{N^{3}}=\frac{|\varepsilon|^{2}\left(1+4|\varepsilon|^{2}+|\varepsilon|^{4}\right)}{\left(1-|\varepsilon|^{2}\right)^{3}}, \quad \overline{(\Delta N)^{3}}=\frac{|\varepsilon|^{2}\left(1+|\varepsilon|^{2}\right)}{\left(1-|\varepsilon|^{2}\right)^{3}}
\end{gathered}
$$

\begin{tabular}{|c|c|c|c|c|c|c|c|c|c|c|}
\hline$k$ & $E_{1}(k, 0)$ & $E_{1}(k, 1)$ & $E_{1}(k, 2)$ & $E_{1}(k, 3)$ & $E_{1}(k, 4)$ & $E_{1}(k, 5)$ & $E_{1}(k, 6)$ & $E_{1}(k, 7)$ & $E_{1}(k, 8)$ & $\sum_{l=0}^{k} E_{1}(k, l)$ \\
\hline 0 & 1 & & & & & & & & & $1=0 !$ \\
\hline 1 & 1 & & & & & & & & & $1=1 !$ \\
\hline 2 & 1 & 1 & & & & & & & & $2=2 !$ \\
\hline 3 & 1 & 4 & 1 & & & & & & & $6=3 !$ \\
\hline 4 & 1 & 11 & 11 & 1 & & & & & & $24=4 !$ \\
\hline 5 & 1 & 26 & 66 & 26 & 1 & & & & & $120=5 !$ \\
\hline 6 & 1 & 57 & 302 & 302 & 57 & 1 & & & & $720=6 !$ \\
\hline 7 & 1 & 120 & 1191 & 2416 & 1191 & 120 & 1 & & & $5040=7 !$ \\
\hline 8 & 1 & 247 & 4293 & 15619 & 15619 & 4293 & 247 & 1 & & $40320=8 !$ \\
\hline 9 & 1 & 502 & 14608 & 88234 & 156190 & 88234 & 14608 & 502 & 1 & $362880=9 !$ \\
\hline
\end{tabular}

For $\overline{(\Delta N)^{2}}-\bar{N}$ which by definition is the boundary between sub- and super-Poissonian statistics we find

$$
\overline{(\Delta N)^{2}}-\bar{N}=\overline{a^{\dagger 2} a^{2}}-{\overline{a^{\dagger} a}}^{2}=\left(\frac{|\varepsilon|^{2}}{1-|\varepsilon|^{2}}\right)^{2} \geq 0 .
$$

Table 1. Eulerian numbers $E_{1}(k, l)$. 
This shows that among the coherent phase states are not states with sub-Poissonian statistics for which by definition should hold $\overline{a^{\dagger 2} a^{2}}-\overline{a^{\dagger} a}<0$. The measure $\overline{(\Delta N)^{3}}$ shows that the coherent phase states possess for $|\varepsilon| \rightarrow 1$ a great asymmetry around the mean values $(\bar{Q}, \bar{P})$. Furthermore from (12.2) follows

$$
2 \bar{N}+1=\overline{a a^{\dagger}+a^{\dagger} a}=\frac{1+|\varepsilon|^{2}}{1-|\varepsilon|^{2}} .
$$

We use this result in Section 13 in the calculation of $\overline{(\Delta Q)^{2}}$ and $\overline{(\Delta P)^{2}}$.

The Hilbert-Schmidt distance to the vacuum state with density operator $\rho=|0\rangle\langle 0|$ is

$$
\begin{aligned}
& d_{\mathrm{HS}}(|\varepsilon\rangle\langle\varepsilon|,| 0\rangle\langle 0|) \equiv \sqrt{\left\langle(|\varepsilon\rangle\langle\varepsilon|-| 0\rangle\langle 0|)^{2}\right\rangle} \\
& =\sqrt{2\left\{1-\left(1-|\varepsilon|^{2}\right)\right\}}=\sqrt{2}|\varepsilon| \leq \sqrt{2 \bar{N}} .
\end{aligned}
$$

For $|\varepsilon| \rightarrow 1$ the Hilbert-Schmidt distance [24] [25] to the vacuum state goes to the maximally possible ones equal to $\sqrt{2}$. The Hilbert-Schmidt distance of $|\varepsilon\rangle$ to an arbitrary coherent state $|\alpha\rangle$ is

$$
\begin{aligned}
& d_{\mathrm{HS}}(|\varepsilon\rangle\langle\varepsilon|,| \alpha\rangle\langle\alpha|)=\sqrt{2\{1-\langle\varepsilon \mid \alpha\rangle\langle\alpha \mid \varepsilon\rangle\}} \\
& =\sqrt{2\left\{1-\left(1-\varepsilon \varepsilon^{*}\right) \exp \left(-\alpha \alpha^{*}\right) f\left(\varepsilon \alpha^{*}\right) f\left(\varepsilon^{*} \alpha\right)\right\}} .
\end{aligned}
$$

The minimal distance to a coherent state as possible measure of non-classicality of a state [25] is obtained if $\varepsilon$ and $\alpha$ possess the same phase and is then determined by the minimum of (12.6) or the maximum of $\exp \left(-|\alpha|^{2}\right) f(|\varepsilon||\alpha|)^{2}=\exp \left(-\left(1-|\varepsilon|^{2}\right)|\alpha|^{2}\right) f_{0}(|\varepsilon||\alpha|)^{2} \quad$ with respect to variation of $|\alpha|$. Due to the difficulties with the unorthodox function $f(z)$ it is not easy to derive approximations. For $|\varepsilon| \rightarrow 1$ the minimal distance (12.6) goes to $\sqrt{2}$ that means to the maximal possible one for normalized states.

\section{Expectation Values for Canonical Operators $Q$ and $P$ and Their Variances for Coherent Phase States}

In this Section we discuss the calculation of expectation values of the canonical operators $Q$ and $P$ and of simple functions of these operators for coherent phase states $|\varepsilon\rangle$.

We begin with the calculation of the expectation value of the annihilation operator $a$ for which we find from (8.4) the following series

$$
\begin{aligned}
\bar{a} & \equiv\langle\varepsilon|a| \varepsilon\rangle=\left(1-\varepsilon \varepsilon^{*}\right) \sum_{m=0}^{\infty} \sum_{n=0}^{\infty} \varepsilon^{m} \varepsilon^{*_{n}}\langle n|a| m\rangle \\
& =\left(1-|\varepsilon|^{2}\right) \sum_{m=0}^{\infty} \sum_{n=0}^{\infty} \varepsilon^{m} \varepsilon^{*_{n}} \sqrt{n+1}\langle n+1 \mid m\rangle \\
& =\varepsilon\left(1-|\varepsilon|^{2}\right) \sum_{n=0}^{\infty} \sqrt{n+1}|\varepsilon|^{2 n}, \quad \overline{a^{\dagger}}=\bar{a}^{*} .
\end{aligned}
$$

In the same way we find for the expectation value of the operator $a^{2}$ 


$$
\begin{aligned}
\overline{a^{2}} & \equiv\left\langle\varepsilon\left|a^{2}\right| \varepsilon\right\rangle=\left(1-\varepsilon \varepsilon^{*}\right) \sum_{m=0}^{\infty} \sum_{n=0}^{\infty} \varepsilon^{m} \varepsilon^{* n}\left\langle n\left|a^{2}\right| m\right\rangle \\
& =\left(1-|\varepsilon|^{2}\right) \sum_{m=0}^{\infty} \sum_{n=0}^{\infty} \varepsilon^{m} \varepsilon^{* n} \sqrt{(n+2)(n+1)}\langle n+2 \mid m\rangle \\
& =\varepsilon^{2}\left(1-|\varepsilon|^{2}\right) \sum_{n=0}^{\infty} \sqrt{(n+2)(n+1)}|\varepsilon|^{2 n}, \quad \overline{a^{\dagger 2}}={\overline{a^{2}}}^{*} .
\end{aligned}
$$

The right-hand sides of (13.1) and (13.2) are series roughly speaking of the type of a Geometric series $G_{1}(0, z) \equiv \sum_{n=0}^{\infty} z^{n}=(1-z)^{-1}$, however, with additional slowly increasing coefficients in front of $z^{n}$ which we more generally denoted and evaluated in [16] by

$$
G_{\mu}(k ; z) \equiv \sum_{n=0}^{\infty}\left(\frac{(n+\mu) !}{n !}\right)^{k} z^{n} \equiv \frac{E_{\mu}(k ; z)}{(1-z)^{\mu k+1}} \equiv \frac{1}{(1-z)^{\mu k+1}} \sum_{l=0}^{\mu k} E_{\mu}(k, l) z^{l} .
$$

The numbers $E_{\mu}(k, l)$ are generalized Eulerian numbers for which we found the general formula

$$
E_{\mu}(k, l) \equiv \sum_{j=0}^{l} \frac{(-1)^{j}(\mu k+1) !}{j !(\mu k+1-j) !}\left(\frac{(l-j+\mu) !}{(l-j) !}\right)^{k}
$$

We see that the sum (13.1) is of the type $G_{1}\left(\frac{1}{2}, z\right)$ and (13.2) of the type $G_{2}\left(\frac{1}{2}, z\right)$ of a Generalized Geometric series where $k=\frac{1}{2}$ is not an integer and, therefore, where the Generalized Eulerian numbers $E_{1}\left(\frac{1}{2}, l\right)$ according to (13.4) are non-vanishing for arbitrary $l=0,1,2, \cdots$. For integer $k$ the Eulerian numbers $E_{1}(k, l)$ are different from zero only for $l \leq k$ as shown in preceding Section [16] [17] [18]. Thus we may write the result of evaluation of (13.1) in the form

$$
\bar{a}=\frac{\varepsilon}{\sqrt{1-|\varepsilon|^{2}}} \sum_{l=0}^{\infty} E_{1}\left(\frac{1}{2}, l\right)|\varepsilon|^{2 l}, \quad \overline{a^{\dagger}}=\bar{a}^{*}
$$

with

$$
\begin{aligned}
& E_{1}\left(\frac{1}{2}, l\right)=\sum_{j=0}^{l} \frac{(-1)^{j}\left(\frac{3}{2}\right) !}{j !\left(\frac{3}{2}-j\right) !} \sqrt{l+1-j}, \\
& \sum_{l=0}^{\infty} E_{1}\left(\frac{1}{2}, l\right)=\left(\frac{1}{2}\right) !=\frac{\sqrt{\pi}}{2} \approx 0.886227,
\end{aligned}
$$

and the result of evaluation of (13.2) in the form

$$
\overline{a^{2}}=\frac{\varepsilon^{2}}{1-|\varepsilon|^{2}} \sum_{l=0}^{\infty} E_{2}\left(\frac{1}{2}, l\right)|\varepsilon|^{2 l}, \quad \overline{a^{\dagger}}=\bar{a}^{*},
$$

with 
$E_{2}\left(\frac{1}{2}, l\right)=\sum_{j=0}^{l} \frac{(-1)^{j} 2 !}{j !(2-j) !} \sqrt{(l+2-j)(l+1-j)}, \quad \sum_{l=0}^{\infty} E_{2}\left(\frac{1}{2}, l\right)=\left(2 \frac{1}{2}\right) !=1$.

The splitting of the functions $(1-z)^{-\frac{3}{2}}$ in our case from $G_{1}\left(\frac{1}{2}, z\right)$ and of $(1-z)^{-2}$ from $G_{2}\left(\frac{1}{2}, z\right)$ is very important for the acceleration of the convergence of series in the evaluation, in particular, for $|z|<1$ in the neighborhood of $|z| \simeq 1$ and for approximate solutions it is then necessary to take into account only a few number of terms of the series $E_{1}\left(\frac{1}{2}, z\right)$ or $E_{2}\left(\frac{1}{2}, z\right)$. With a few terms of the expansion we find for the series involved in (13.5)

$$
\begin{aligned}
& \sum_{l=0}^{\infty} E_{1}\left(\frac{1}{2}, l\right)|\varepsilon|^{2 l} \\
& =1-\left(\frac{3}{2}-\sqrt{2}\right)|\varepsilon|^{2}-\left(\frac{3}{\sqrt{2}}-\frac{3}{8}-\sqrt{3}\right)|\varepsilon|^{4}-\left(\frac{3 \sqrt{3}}{2}-\frac{33}{16}-\frac{3}{4 \sqrt{2}}\right)|\varepsilon|^{6}-\cdots \\
& =1-0.0857864|\varepsilon|^{2}-0.0142695|\varepsilon|^{4}-0.00524613|\varepsilon|^{6}-\cdots
\end{aligned}
$$

satisfying for $|\varepsilon| \leq 1$ the inequality

$$
\frac{\sqrt{\pi}}{2} \leq \sum_{l=0}^{\infty} E_{1}\left(\frac{1}{2}, l\right)|\varepsilon|^{2 l} \leq 1, \quad(|\varepsilon|<1),
$$

and for the series involved in (13.7)

$$
\begin{aligned}
& \sum_{l=0}^{\infty} E_{2}\left(\frac{1}{2}, l\right)|\varepsilon|^{2 l} \\
& =\sqrt{2}-(2 \sqrt{2}-\sqrt{6})|\varepsilon|^{2}-(2 \sqrt{6}-\sqrt{2}-2 \sqrt{3})|\varepsilon|^{4}-(4 \sqrt{3}-2 \sqrt{5}-\sqrt{6})|\varepsilon|^{6}-\cdots \\
& =1.41421-0.378937|\varepsilon|^{2}-0.0206643|\varepsilon|^{4}-0.00657753|\varepsilon|^{6}-\cdots
\end{aligned}
$$

satisfying for $|\varepsilon| \leq 1$ the inequality

$$
1 \leq \sum_{l=0}^{\infty} E_{2}\left(\frac{1}{2}, l\right)|\varepsilon|^{2 l} \leq \sqrt{2} . \quad(|\varepsilon|<1) .
$$

In the following we need also the square of the expression (13.9) for which we find the first sum terms of the expansion

$$
\begin{aligned}
& \left(\sum_{l=0}^{\infty} E_{1}\left(\frac{1}{2}, l\right)|\varepsilon|^{2 l}\right)^{2} \\
& =1-(3-2 \sqrt{2})|\varepsilon|^{2}-(6 \sqrt{2}-5-2 \sqrt{3})|\varepsilon|^{4}-(6 \sqrt{3}+3-6 \sqrt{2}-2 \sqrt{6})|\varepsilon|^{6}-\cdots \\
& =1-0.171573|\varepsilon|^{2}-0.0211798|\varepsilon|^{4}-0.00804399|\varepsilon|^{6}-\cdots
\end{aligned}
$$

As an interesting observation we remark that in contrast to the expectation values $\bar{Q}$ and $\bar{P}$ in (13.13) in the coefficients in braces we do not have denominators different from 1 in the corresponding expansions for the squared 
expectation values $\bar{Q}^{2}$ and $\bar{P}^{2}$. We checked that this is true even up to $25 \mathrm{sum}$ terms in the approximation but do not try to generalize it for arbitrary order and to prove it. Although this seems to be unimportant for numerical calculations it is perhaps interesting for number-theoretic considerations (likely, this is connected with $k=\frac{1}{2}$ in the generalized Eulerian numbers $E_{1}(k, l)$ that their squares possess the mentioned property).

For the expectation values of the canonical operators $Q$ and $P$ we find

$$
\begin{gathered}
\bar{Q}=\sqrt{\frac{\hbar}{2}}\left(\bar{a}+\overline{a^{\dagger}}\right)=\sqrt{\frac{\hbar}{2}} \frac{\varepsilon+\varepsilon^{*}}{\sqrt{1-|\varepsilon|^{2}}} \sum_{l=0}^{\infty} E_{1}\left(\frac{1}{2}, l\right)|\varepsilon|^{2 l}, \\
\bar{P}=-\mathrm{i} \sqrt{\frac{\hbar}{2}}\left(\bar{a}-\overline{a^{\dagger}}\right)=-\mathrm{i} \sqrt{\frac{\hbar}{2}} \frac{\varepsilon-\varepsilon^{*}}{\sqrt{1-|\varepsilon|^{2}}} \sum_{l=0}^{\infty} E_{1}\left(\frac{1}{2}, l\right)|\varepsilon|^{2 l} .
\end{gathered}
$$

For their squares we calculate

$$
\begin{aligned}
& \bar{Q}^{2}=\frac{\hbar}{2} \frac{2 \varepsilon \varepsilon^{*}+\varepsilon^{2}+\varepsilon^{* 2}}{1-|\varepsilon|^{2}}\left(\sum_{l=0}^{\infty} E_{1}\left(\frac{1}{2}, l\right)|\varepsilon|^{2 l}\right)^{2}, \\
& \bar{P}^{2}=\frac{\hbar}{2} \frac{2 \varepsilon \varepsilon^{*}-\varepsilon^{2}-\varepsilon^{* 2}}{1-|\varepsilon|^{2}}\left(\sum_{l=0}^{\infty} E_{1}\left(\frac{1}{2}, l\right)|\varepsilon|^{2 l}\right)^{2},
\end{aligned}
$$

and for their product

$$
\bar{Q} \bar{P}=-\mathrm{i} \frac{\hbar}{2} \frac{\varepsilon^{2}-\varepsilon^{* 2}}{1-|\varepsilon|^{2}}\left(\sum_{l=0}^{\infty} E_{1}\left(\frac{1}{2}, l\right)|\varepsilon|^{2 l}\right)^{2} .
$$

We come now to the expectation values of the operators $Q^{2}$ and $P^{2}$ for which we find using (13.7)

$$
\begin{gathered}
\overline{Q^{2}}=\frac{\hbar}{2}\left(\overline{a a^{\dagger}+a^{\dagger} a}+\overline{a^{2}+a^{\dagger 2}}\right)=\frac{\hbar}{2}\left\{\frac{1+|\varepsilon|^{2}}{1-|\varepsilon|^{2}}+\frac{\varepsilon^{2}+\varepsilon^{* 2}}{1-|\varepsilon|^{2}} \sum_{l=0}^{\infty} E_{2}\left(\frac{1}{2}, l\right)|\varepsilon|^{2 l}\right\}, \\
\overline{P^{2}}=\frac{\hbar}{2}\left(\overline{a a^{\dagger}+a^{\dagger} a}-\overline{a^{2}+a^{\dagger 2}}\right)=\frac{\hbar}{2}\left\{\frac{1+|\varepsilon|^{2}}{1-|\varepsilon|^{2}}-\frac{\varepsilon^{2}+\varepsilon^{* 2}}{1-|\varepsilon|^{2}} \sum_{l=0}^{\infty} E_{2}\left(\frac{1}{2}, l\right)|\varepsilon|^{2 l}\right\} .
\end{gathered}
$$

We inserted here the result (12.4) for the expectation value of $\overline{a a^{\dagger}+a^{\dagger} a}$. The lastly needed expectation value of quadratic combinations of the canonical operators $Q$ and $P$ is

$$
\begin{aligned}
\frac{1}{2} \overline{Q P+P Q} & =-\mathrm{i} \frac{\hbar}{2} \overline{a^{2}-a^{\dagger 2}} \\
& =-\mathrm{i} \frac{\hbar}{2} \frac{\varepsilon^{2}-\varepsilon^{* 2}}{1-|\varepsilon|^{2}} \sum_{l=0}^{\infty} E_{2}\left(\frac{1}{2}, l\right)|\varepsilon|^{2 l}
\end{aligned}
$$

This expression is vanishing for $\varepsilon= \pm \varepsilon^{*}$ that means if $\varepsilon$ is a real or an imaginary number. In all other cases of complex numbers $\varepsilon$ it is non-vanishing.

Using the results of this Section we calculate in next Section variances to the expectation values of the canonical operators. 


\section{Uncertainties, Uncertainty Matrix and Uncertainty Correlations}

The uncertainties of the expectation values of the canonical operators emphasizing their transformation and invariance properties are well summarized in the following symmetric uncertainty matrix $S$ (e.g., [39])

$$
\boldsymbol{S}=\left(\begin{array}{cc}
\overline{(\Delta Q)^{2}}, & \frac{1}{2} \overline{\Delta Q \Delta P+\Delta P \Delta Q} \\
\frac{1}{2} \overline{\Delta Q \Delta P+\Delta P \Delta Q}, & \overline{(\Delta P)^{2}}
\end{array}\right),
$$

where $\Delta Q=Q-\bar{Q} I$ and $\Delta P=P-\bar{P} I$ and expectation values $\bar{A}$ are defined by $\bar{A}=\langle\varrho A\rangle$ with $\varrho$ the density operator of the considered state. In the preceding Section we prepared the calculation of this uncertainty matrix for coherent phase states with density operator $\varrho=|\varepsilon\rangle\langle\varepsilon|$ by the calculation of partial building stones.

By combination of the results in Equations (13.17) and (13.15) we find for the variances $\overline{(\Delta Q)^{2}}$ and $\overline{(\Delta P)^{2}}$

$$
\begin{aligned}
& \overline{(\Delta Q)^{2}} \equiv \overline{Q^{2}}-\bar{Q}^{2}=\frac{\hbar}{2}\left\{\frac{1+|\varepsilon|^{2}}{1-|\varepsilon|^{2}}-\frac{2|\varepsilon|^{2}}{1-|\varepsilon|^{2}}\left(\sum_{l=0}^{\infty} E_{1}\left(\frac{1}{2}, l\right)|\varepsilon|^{2 l}\right)^{2}\right. \\
&\left.+\frac{\varepsilon^{2}+\varepsilon^{* 2}}{1-|\varepsilon|^{2}}\left(\sum_{l=0}^{\infty} E_{2}\left(\frac{1}{2}, l\right)|\varepsilon|^{2 l}-\left(\sum_{l=0}^{\infty} E_{1}\left(\frac{1}{2}, l\right)|\varepsilon|^{2 l}\right)^{2}\right)\right\}, \\
& \overline{(\Delta P)^{2} \equiv} \equiv \overline{P^{2}}-\bar{P}^{2}= \\
& \frac{\hbar}{2}\left\{\frac{1+|\varepsilon|^{2}}{1-|\varepsilon|^{2}}-\frac{2|\varepsilon|^{2}}{1-|\varepsilon|^{2}}\left(\sum_{l=0}^{\infty} E_{1}\left(\frac{1}{2}, l\right)|\varepsilon|^{2 l}\right)^{2}\right. \\
&\left.-\frac{\varepsilon^{2}+\varepsilon^{* 2}}{1-|\varepsilon|^{2}}\left(\sum_{l=0}^{\infty} E_{2}\left(\frac{1}{2}, l\right)|\varepsilon|^{2 l}-\left(\sum_{l=0}^{\infty} E_{1}\left(\frac{1}{2}, l\right)|\varepsilon|^{2 l}\right)^{2}\right)\right\},
\end{aligned}
$$

and by combination of (13.18) and (13.16) for the uncertainty correlation $\frac{1}{2} \overline{\Delta Q \Delta P+\Delta P \Delta Q}$

$$
\begin{aligned}
& \frac{1}{2} \overline{\Delta Q \Delta P+\Delta P \Delta Q}=\frac{1}{2} \overline{Q P+P Q}-\bar{Q} \bar{P} \\
& =-\mathrm{i} \frac{\hbar}{2} \frac{\varepsilon^{2}-\varepsilon^{* 2}}{1-|\varepsilon|^{2}}\left(\sum_{l=0}^{\infty} E_{2}\left(\frac{1}{1}, l\right)|\varepsilon|^{2 l}-\left(\sum_{l=0}^{\infty} E_{1}\left(\frac{1}{1}, l\right)|\varepsilon|^{2 l}\right)^{2}\right) .
\end{aligned}
$$

We find the following few first terms of expansions of common partial expressions in (14.2) and (14.3)

$$
\begin{aligned}
\sum_{l=0}^{\infty} E_{2}\left(\frac{1}{2}, l\right)|\varepsilon|^{2 l}-\left(\sum_{l=0}^{\infty} E_{1}\left(\frac{1}{2}, l\right)|\varepsilon|^{2 l}\right)^{2} \\
=\sqrt{2}-1-(4 \sqrt{2}-3-\sqrt{6})|\varepsilon|^{2}+(7 \sqrt{2}-5-2 \sqrt{6})|\varepsilon|^{4} \\
+(3+2 \sqrt{3}+2 \sqrt{5}-6 \sqrt{2}-\sqrt{6})|\varepsilon|^{6}+\cdots \\
=0.414214-0.207365|\varepsilon|^{2}+0.000515451|\varepsilon|^{4}+0.00146645|\varepsilon|^{6}+\cdots
\end{aligned}
$$


The corresponding expansion for the expression $\left(\sum_{l=1}^{\infty} E_{1}\left(\frac{1}{2}, l\right)|\varepsilon|^{2 l}\right)^{2}$ was already given in (13.13).

The uncertainty sum is the trace $\langle\boldsymbol{S}\rangle$ of the uncertainty matrix $S$ and we find

$$
\begin{aligned}
\langle\boldsymbol{S}\rangle & \equiv \overline{(\Delta Q)^{2}}+\overline{(\Delta P)^{2}} \\
& =\hbar\left\{\frac{1+|\varepsilon|^{2}}{1-|\varepsilon|^{2}}-\frac{2|\varepsilon|^{2}}{1-|\varepsilon|^{2}}\left(\sum_{l=0}^{\infty} E_{1}\left(\frac{1}{2}, l\right)|\varepsilon|^{2 l}\right)^{2}\right\} .
\end{aligned}
$$

It depends only on the modulus $|\varepsilon|$ of the complex $\varepsilon=|\varepsilon| \mathrm{e}^{\mathrm{i} \varphi}$ but does not depend on the angle $\varphi$. Thus it is invariant with respect to rotations of the coherent phase states in the phase plane of variables $(q, p)$. In the limiting case $|\varepsilon| \rightarrow 0$ using (13.13) and in the limiting case $|\varepsilon| \rightarrow 1$ we obtain

$$
\lim _{|\varepsilon| \rightarrow 0}\left(\overline{(\Delta Q)^{2}}+\overline{(\Delta P)^{2}}\right)=\hbar, \quad \lim _{|\varepsilon| \rightarrow 1}\left(\overline{(\Delta Q)^{2}}+\overline{(\Delta P)^{2}}\right) \rightarrow \infty .
$$

The second invariant of the two-dimensional matrix $S$ with respect to similarity transformations is its determinant $[\boldsymbol{S}]$ which provides here the uncertainty product taking into account the uncertainty correlations for which we find using (13.17) and (13.18) together with (13.15) and (13.16)

$$
\begin{aligned}
{[\boldsymbol{S}] \equiv } & \overline{(\Delta Q)^{2}(\Delta P)^{2}}-\frac{1}{4}(\overline{\Delta Q \Delta P+\Delta P \Delta Q})^{2} \\
= & \frac{\hbar^{2}}{4}\left\{\left(\frac{1+|\varepsilon|^{2}}{1-|\varepsilon|^{2}}-\frac{2|\varepsilon|^{2}}{1-|\varepsilon|^{2}}\left(\sum_{l=0}^{\infty} E_{1}\left(\frac{1}{2}, l\right)|\varepsilon|^{2 l}\right)^{2}\right)^{2}\right. \\
& \left.-\left(\frac{2|\varepsilon|^{2}}{1-|\varepsilon|^{2}}\right)^{2}\left(\sum_{l=0}^{\infty} E_{2}\left(\frac{1}{2}, l\right)|\varepsilon|^{2 l}-\left(\sum_{l=0}^{\infty} E_{1}\left(\frac{1}{2}, l\right)|\varepsilon|^{2 l}\right)^{2}\right)^{2}\right\} .
\end{aligned}
$$

In the same way as the uncertainty sum $\langle\boldsymbol{S}\rangle$ it depends only on the modulus of $\varepsilon$ but not on its angle. If we apply the binomial formula to the two quadratic terms in (14.7) then the last two terms cancel but we do not come in this way to an essentially shorter representation. We come back to this below. In the limiting cases we find

$$
\begin{aligned}
& \lim _{|\varepsilon| \rightarrow 0}\left(\overline{(\Delta Q)^{2}(\Delta P)^{2}}-\frac{1}{4}(\overline{\Delta Q \Delta P+\Delta P \Delta Q})^{2}\right)=\frac{\hbar^{2}}{4}, \\
& \lim _{|\varepsilon| \rightarrow 1}\left(\overline{(\Delta Q)^{2}(\Delta P)^{2}}-\frac{1}{4}(\overline{\Delta Q \Delta P+\Delta P \Delta Q})^{2}\right) \rightarrow \infty .
\end{aligned}
$$

The most expressions which we may calculate from $Q$ and $P$ are not invariant with respect to the phase of the complex parameter $\varepsilon$. For example, the mean values $\bar{Q}$ and $\bar{P}$ depend on the phase of $\varepsilon$. If we choose

$$
\varepsilon=\varepsilon^{*}=|\varepsilon|,
$$

then we find 


$$
\bar{Q}=\sqrt{\frac{\hbar}{2}} \frac{2|\varepsilon|}{\sqrt{1-|\varepsilon|^{2}}} \sum_{l=0}^{\infty} E_{1}\left(\frac{1}{2}, l\right)|\varepsilon|^{2 l}, \quad \bar{P}=0 .
$$

The vector of the mean values $(\bar{Q}, \bar{P})$ lies on the positive $q$-axis and continuously increases there with increasing $|\varepsilon|<1$. For the squares we find

$$
\bar{Q}^{2}=\frac{\hbar}{2} \frac{4|\varepsilon|^{2}}{1-|\varepsilon|^{2}}\left(\sum_{l=0}^{\infty} E_{1}\left(\frac{1}{2}, l\right)|\varepsilon|^{2 l}\right)^{2}, \quad \bar{P}^{2}=0,
$$

and for the expectation values $\overline{Q^{2}}$ and $\overline{P^{2}}$

$$
\begin{aligned}
& \overline{Q^{2}}=\frac{\hbar}{2}\left\{\frac{1+|\varepsilon|^{2}}{1-|\varepsilon|^{2}}+\frac{2|\varepsilon|^{2}}{1-|\varepsilon|^{2}} \sum_{l=0}^{\infty} E_{2}\left(\frac{1}{2}, l\right)|\varepsilon|^{2 l}\right\}, \\
& \overline{P^{2}}=\frac{\hbar}{2}\left\{\frac{1+|\varepsilon|^{2}}{1-|\varepsilon|^{2}}-\frac{2|\varepsilon|^{2}}{1-|\varepsilon|^{2}} \sum_{l=0}^{\infty} E_{2}\left(\frac{1}{2}, l\right)|\varepsilon|^{2 l}\right\} .
\end{aligned}
$$

For the corresponding variances we now obtain the formally asymmetric expressions for $\overline{(\Delta Q)^{2}}$ and $\overline{(\Delta P)^{2}}$

$$
\begin{gathered}
\overline{(\Delta Q)^{2}}=\frac{\hbar}{2}\left\{\frac{1+|\varepsilon|^{2}}{1-|\varepsilon|^{2}}+\frac{2|\varepsilon|^{2}}{1-|\varepsilon|^{2}}\left(\sum_{l=0}^{\infty} E_{2}\left(\frac{1}{2}, l\right)|\varepsilon|^{2 l}-2\left(\sum_{l=0}^{\infty} E_{1}\left(\frac{1}{2}, l\right)|\varepsilon|^{2 l}\right)^{2}\right)\right\}, \\
\overline{(\Delta P)^{2}}=\frac{\hbar}{2}\left\{\frac{1+|\varepsilon|^{2}}{1-|\varepsilon|^{2}}-\frac{2|\varepsilon|^{2}}{1-|\varepsilon|^{2}} \sum_{l=0}^{\infty} E_{2}\left(\frac{1}{2}, l\right)|\varepsilon|^{2 l}\right\} .
\end{gathered}
$$

The uncertainty correlation (14.3) vanishes in the special case (14.9) that means

$$
\overline{\triangle Q \Delta P+\Delta P \Delta Q}=0,
$$

and the matrix (14.1) becomes diagonal. The uncertainty sum is invariant with respect to rotations of $\varepsilon$ and therefore we find from (14.13) again the expression (14.5). For the uncertainty product in the special case (14.9) follows from (14.13)

$$
\begin{aligned}
\overline{(\Delta Q)^{2}}(\Delta P)^{2} \\
=\frac{\hbar^{2}}{4}\left\{\left(\frac{1+|\varepsilon|^{2}}{1-|\varepsilon|^{2}}\right)^{2}-\left(\frac{2|\varepsilon|^{2}}{1-|\varepsilon|^{2}} \sum_{l=0}^{\infty} E_{2}\left(\frac{1}{2}, l\right)|\varepsilon|^{2 l}\right)^{2}\right. \\
\left.-\frac{4|\varepsilon|^{2}}{1-|\varepsilon|^{2}}\left(\sum_{l=0}^{\infty} E_{1}\left(\frac{1}{2}, l\right)|\varepsilon|^{2 l}\right)^{2}\left(\frac{1+|\varepsilon|^{2}}{1-|\varepsilon|^{2}}-\frac{2|\varepsilon|^{2}}{1-|\varepsilon|^{2}} \sum_{l=0}^{\infty} E_{2}\left(\frac{1}{2}, l\right)|\varepsilon|^{2 l}\right)\right\} .
\end{aligned}
$$

This is the same as (14.7) in an alternative representation but in the considered special case (14.9) the uncertainty correlation vanishes. In general, the uncertainty product is not invariant with respect of rotations of the state in the phase space or here with respect to the phase of $\varepsilon$. The uncertainties obey the following general chain of inequalities [39] 


$$
\begin{aligned}
\frac{\hbar}{2} & \leq \sqrt{[S]} \equiv \sqrt{\overline{(\Delta Q)^{2}}(\Delta P)^{2}}-\frac{1}{4}(\overline{\Delta Q \Delta P+\Delta P \Delta Q})^{2} \\
& \leq \sqrt{\overline{(\Delta Q)^{2}(\Delta P)^{2}}} \leq \frac{1}{2}\langle\boldsymbol{S}\rangle \equiv \frac{1}{2}\left(\overline{(\Delta Q)^{2}}+\overline{(\Delta P)^{2}}\right) .
\end{aligned}
$$

As known, in general, the minimal uncertainty product for a given state is only obtained under special positions of the axes $(q, p)$ of the phase space but the uncertainty product taking into account the uncertainty correlations provide it in every case. The inequality between the square of the uncertainty product taking into account the uncertainty correlation and the half uncertainty sum is the inequality between geometric and arithmetic mean. For imaginary parameter $\varepsilon$ instead of real one the roles of the axes are interchanged.

The uncertainties for coherent phase states $|\varepsilon\rangle$ are graphically represented in Figure 6.

\section{Conclusions}

We derived in this article formulae for the calculation of the Wigner quasiprobability with emphasis of relations which are symmetric in the canonical operators $(Q, P)$. We applied them to the eigenstates of the one-dimensional square well with infinitely high impenetrable walls and in quantum optics to the coherent phase states. Both considered examples are quantum-mechanical systems with $S U(1,1)$ symmetry. For the coherent phase states we discussed in detail the difficulties of calculations due to a very unorthodox entire function involved in such calculations. We tried to bring more light in the calculations of properties of coherent phase states and of their Wigner quasiprobability which did not be dealt with up to now in satisfactory form.

An interesting question without a clear answer is whether nature can realize
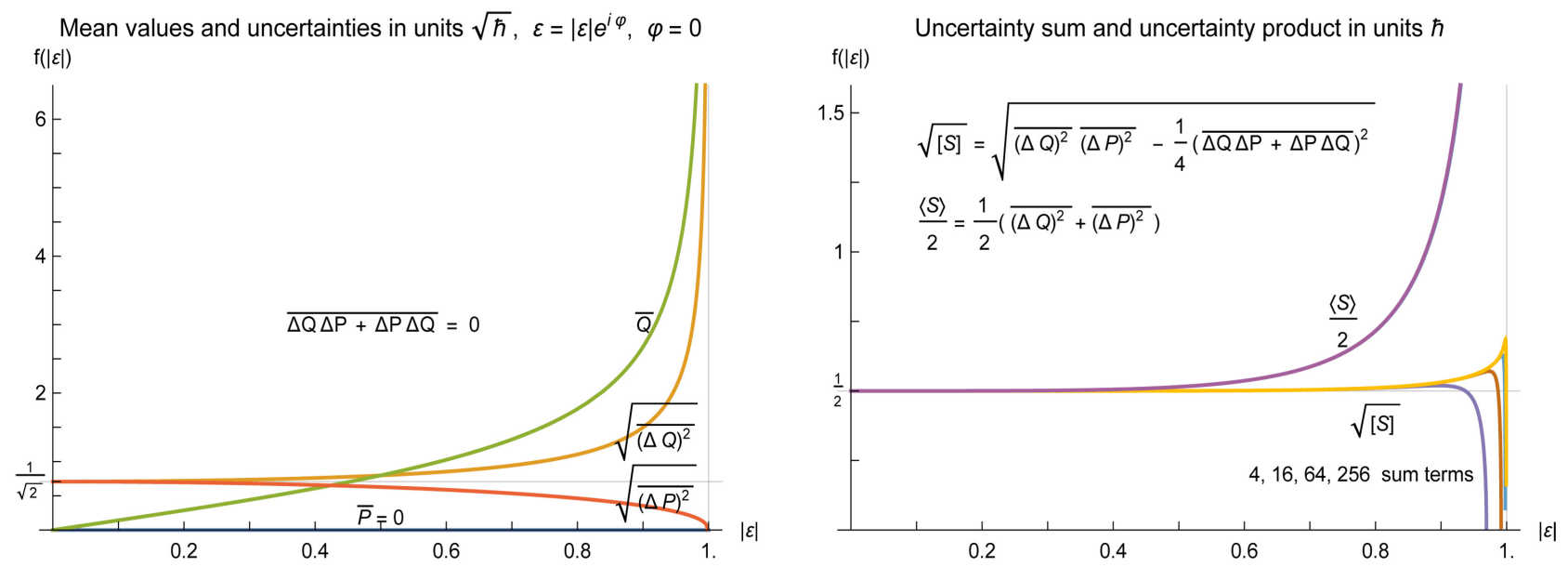

Figure 6. Uncertainties for coherent phase states $|\varepsilon\rangle$. In the partial figure to the left we have chosen $\varphi=0$ in $\varepsilon=|\varepsilon| \mathrm{e}^{\mathrm{i} \varphi}$ and, therefore, $\bar{P}=0$. The partial figure to the right is invariant concerning the choice of $\varphi$. In the lower curve we see 4 different approximations. They are generated with taking into account different numbers of sum terms in the expressions which were 4,16 , 64,256 . With the upper curve it was made the same but here the differences are not to see; ("Mathematica 10" in 2018). 
coherent phase states or not. It is meant by this not only an approximate realization, for example, by superposition of a few coherent states on a line beginning from the center or by squeezed coherent states. The reason as mentioned is that in the description of the genuine coherent phase states a very unorthodox function $\sum_{n=0}^{\infty} \frac{z^{n}}{\sqrt{n !}}$ plays a main role which by digital calculations makes considerable trouble. Is nature really capable to realize this by an analog physical process without such a trouble?

The Wigner quasiprobability is an essential mean for the illustration of the connection of quantum theory with classical mechanics but not in all cases the calculation of properties of states from the Wigner quasiprobability is the most suited and easiest one as the considered examples show (remind that it was first developed for corrections to thermodynamic equilibrium). Interesting in a wider sense is, for example, also the interferences seen in the Wigner quasiprobability for superpositions of coherent states in the regions of their overlapping which, however, could not be considered here. A deeper explanation of the origin of the Wigner quasiprobability in possible formulae for expectation values would be desirable. We did not discuss here the transition from quantum to classical mechanics by transition to the infinite number of commutative irreducible representations of the basic operators $(Q, P)$ of the Heisenberg-Weyl algebra for which the operators $|\alpha\rangle\langle\alpha|$ form appropriate bases in the space $H \times H^{*}$ [13].

Finally, we mention that the Wigner quasiprobability possesses also something which is to feel as a very aesthetical aspect.

\section{Acknowledgements}

S. Skorokhodov sent me a nice e-mail in 2008 (see [40] with essential passages in the footnote in Appendix D) but in preparation of the present work I was no more able with my present computer to open the appended file. I thank him very much. In the following years after 2008 I was involved in other topics and was confronted with other problems and so I only now genuinely returned to this.

\section{References}

[1] Wigner, E. (1932) On the Quantum Correction for Thermodynamic Equilibrium. Physical Review, 40, 749. https://doi.org/10.1103/PhysRev.40.749

[2] Kim, Y.S. and Noz, M.E. (1991) Phase Space Picture of Quantum Mechanics. World Scientific, Singapore. https://doi.org/10.1142/1197

[3] Weyl, H. (1928) Gruppentheorie und Quantenmechamik. Hirzel, Leipzig.

[4] Wünsche, A. (2002) Operator Algebras to General One-Dimensional Quantum-Mechanical Potentials with Discrete Spectrum. Journal of Optics B: Quantum and Semiclassical Optics, 4, 359-379. https://doi.org/10.1088/1464-4266/4/6/301

[5] Glauber, R.J. (1963) Coherent and Incoherent States of the Radiation Field. Physical Review, 131, 2766. https://doi.org/10.1103/PhysRev.131.2766

[6] Klauder, J.R. and Sudarshan, E.C.G. (1968) Fundamentals of Quantum Optics. W. A. Benjamin, New York. 
[7] Peřina, J. (1971) Coherence of Light. Van Nostrand Reinhold Company, London.

[8] Klauder, J.R. and Skagerstam, B.-S. (1985) A Coherent-State Primer. World Scientific Publishing, Singapore, 1-115.

[9] Klauder, J.R. and Skagerstam, B.-S. (1985) Coherent States. World Scientific Publishing, Singapore. https://doi.org/10.1142/0096

[10] Schleich, W.P. (2001) Quantum Optics in Phase Space. Wiley-VCH, Berlin. https://doi.org/10.1002/3527602976

[11] Wünsche, A. (2015) Generating Functions for Products of Laguerre 2D and Hermite 2D Polynomials. Applied Mathematics, 6, 2142-2168. https://doi.org/10.4236/am.2015.612188

[12] Perelomov, A.M. (1977) Generalized Coherent States and Some of Their Applications. Uspekhi Fizicheskih Nauk, 123, 23-55. (In Russian) https://doi.org/10.3367/UFNr.0123.197709b.0023

[13] Perelomov, A. (1986) Generalized Coherent States and Their Application. Springer, Berlin. https://doi.org/10.1007/978-3-642-61629-7

[14] Perelomov, A.M. (1987) Obobstschonnyje kogerentnyje sostoyanya i ikh primenyenyje. Nauka, Moskva.

[15] Herzog, U., Paul, H. and Richter, Th. (1993) Wigner Function of a Phase State. Physica Scripta, T48, 61-65. https://doi.org/10.1088/0031-8949/1993/T48/009

[16] Wünsche, A. (2018) Generalized Eulerian Numbers. Advances of Pure Mathematics, 8, Article ID: 83018. https://doi.org/10.4236/apm.2018.83018

[17] Riordan, J. (1958) An Introduction to Combinatorial Analysis. John Wiley \& Sons, New York

[18] Petersen, T.K. (2015) Eulerian Numbers. Birkhäuser (Springer), New York. https://doi.org/10.1007/978-1-4939-3091-3

[19] Tatarskii, V.I. (1983) The Wigner Representation in Quantum Mechanics. Uspekhi Fiz. Nauk, 139, 587. (In Russian)

[20] Hillery, M., O’Connell, R.F., Scully, M.O. and Wigner, E.P. (1984) Distribution Functions in Physics: Fundamentals. Physics Reports, 106, 121-167. https://doi.org/10.1016/0370-1573(84)90160-1

[21] Vourdas, A. (2004) Quantum Systems with Finite Hilbert Space. Reports on Progress in Physics, 67, 267-320. https://doi.org/10.1088/0034-4885/67/3/R03

[22] Flügge, S. (1999) Practical Quantum Mechanics. Springer, Berlin.

[23] Wünsche, A. (2017) About Classical to Quantum Weyl Correspondence. Applied Mathematics, 7, Article ID: 79975. https://doi.org/10.4236/apm.2017.710034

[24] Wünsche, A. (1995) The Distance to Poissonian Statistics as a Supplementary Measure in Quantum Optics. Journal of Applied Physics B, 60, 119-122.

[25] Dodonov, V.V., Man'ko, O.V., Man'ko, V.I. and Wünsche, A. (2000) Hilbert-Schmidt Distance and Nonclassicality of States in Quantum Optics. Journal of Modern Optics, 47, 633-654. https://doi.org/10.1080/09500340008233385

[26] Susskind, L. and Glogower, J. (1964) Quantum Mechanical Phase and Time Operator. Physics, 1, 49-61. https://doi.org/10.1103/PhysicsPhysiqueFizika.1.49

[27] Carruthers, P. and Nieto, M.M. (1968) Phase and Angle Variables in Quantum Mechanics. Reviews of Modern Physics, 40, 411. https://doi.org/10.1103/RevModPhys.40.411

[28] Man'ko, V.I. (Ed.) (1972) Kogerentnyje costoyaniya v kvantovoi teorii. [Coherent States in Quantum Theory.] Mir, Moskva. 
[29] Peřinová, Vl., Lukš, A. and Peřina, J. (1998) Phase in Optics. World Scientific, Singapore.

[30] Vourdas, A. and Wünsche, A. (1998) Resolutions of the Identity in Terms of Line Integrals of $S U(1,1)$ Coherent States. Journal of Physics A: Mathematical and General, 31, 9341-9352. https://doi.org/10.1088/0305-4470/31/46/024

[31] London, F. (1926) Winkelvariable und Kanonische Transformationen in der Undulationsmechanik. Zeitschrift für Physik, 37, 915-925.

https://doi.org/10.1007/BF01397484

[32] Erdélyi, A. (1953) Higher Transcendental Functions, Vol. 2 Bateman Project. McGraw-Hill, New York.

[33] Vladimirov, V.S. (1971) Uravnyeniya matematicheskoy fiziki. [Equations of Mathematical Physics.] Nauka, Moskva.

[34] Wünsche, A. (1999) Realizations of $S U(1,1)$ by Boson Operators with Application to Phase States. Acta Physica Slovaca, 49, 771-782.

[35] Wünsche, A. (2017) About Squeezed Coherent States in Non-Unitary Approach and Relation to Sub- and Super-Poissonian Statistics. Advances in Pure Mathematics, 7, Article ID: 712044 .

[36] Wünsche, A. (2001) A Class of Phase-Like States. Journal of Optics B: Quantum and Semiclassical Optics, 3, 206-218. https://doi.org/10.1088/1464-4266/3/4/303

[37] Wünsche, A. (2001) An Unorthodox Entire Function. Journal of Computational and Applied Mathematics, 133, 700-701. https://doi.org/10.1016/S0377-0427(00)00728-7

[38] Titchmarsh, E.C. (1939) The Theory of Functions. 2nd Edition, Oxford University Press, Oxford.

[39] Wünsche, A. (2015) Quantum-Mechanical Cumulant Expansions and Their Application to Phase-Space and to Phase Distributions. Physica Scripta, 90, Article ID: 074063. https://doi.org/10.1088/0031-8949/90/7/074063

[40] Skorokhodov, S. (2008) Private Communication 2008. Essential Part in Footnote in Appendix D. 


\section{Appendix A}

Fourier Transformation of Coherent States and Representation of Displaced Parity Operator

For the following we need some auxiliary integrals. By transition to polar (or real) coordinates one finds

$$
\frac{1}{\pi c^{2}} \int \frac{\mathrm{i}}{2} \mathrm{~d} z \wedge \mathrm{d} z^{*} \exp \left(w z^{*}-w^{*} z\right) \exp \left(-\frac{z z^{*}}{c^{2}}\right)=\exp \left(-c^{2} w w^{*}\right),
$$

where $c$ is a parameter. From this one calculates the following more general integrals (see (7.1))

$$
\begin{aligned}
& \frac{1}{\pi c^{2}} \int \frac{\mathrm{i}}{2} \mathrm{~d} z \wedge \mathrm{d} z^{*} \exp \left(w z^{*}-w^{*} z\right) \exp \left(-\frac{z z^{*}}{c^{2}}\right) z^{m} z^{*_{n}} \\
& =\left(-\frac{\partial}{\partial w^{*}}\right)^{m}\left(\frac{\partial}{\partial w}\right)^{n} \frac{1}{\pi c^{2}} \int \frac{\mathrm{i}}{2} \mathrm{~d} z \wedge \mathrm{d} z^{*} \exp \left(w z^{*}-w^{*} z\right) \exp \left(-\frac{z z^{*}}{c^{2}}\right) \\
& =(-1)^{m} \frac{\partial^{m+n}}{\partial w^{*} m} \partial w^{n} \exp \left(-c^{2} w w^{*}\right) \\
& =\exp \left(-c^{2} w w^{*}\right)(-1)^{n} c^{m+n} \mathrm{~L}_{m, n}\left(c w, c w^{*}\right) .
\end{aligned}
$$

With the substitutions $w \rightarrow \frac{w}{2}, w^{*} \rightarrow \frac{w^{*}}{2}, c \rightarrow \sqrt{2} b$ one obtains the special cases

$$
\begin{aligned}
& \int \frac{\mathrm{i}}{2} \mathrm{~d} z \wedge \mathrm{d} z^{*} \exp \left(\frac{w z^{*}-w^{*} z}{2}\right) \exp \left(-\frac{z z^{*}}{2 b^{2}}\right) z^{m}=2 \pi b^{2} \exp \left(-\frac{b^{2} w w^{*}}{2}\right)\left(b^{2} w\right)^{m}, \\
& \int \frac{\mathrm{i}}{2} \mathrm{~d} z \wedge \mathrm{d} z^{*} \exp \left(\frac{w z^{*}-w^{*} z}{2}\right) \exp \left(-\frac{z z^{*}}{2 b^{2}}\right) z^{*_{n}}=2 \pi b^{2} \exp \left(-\frac{b^{2} w w^{*}}{2}\right)\left(-b^{2} w^{*}\right)^{n} \text {. }
\end{aligned}
$$

We apply this now.

Using the first formula in (A.3) with $b=1$ one calculates the Fourier transform of a coherent state according to

$$
\begin{aligned}
& \frac{1}{2 \pi} \int \frac{\mathrm{i}}{2} \mathrm{~d} \alpha \wedge \mathrm{d} \alpha^{*} \exp \left(\frac{\beta \alpha^{*}-\beta^{*} \alpha}{2}\right)|\alpha\rangle \\
& =\sum_{n=0}^{\infty} \frac{|n\rangle}{\sqrt{n !}} \frac{1}{2 \pi} \int \frac{\mathrm{i}}{2} \mathrm{~d} \alpha \wedge \mathrm{d} \alpha^{*} \exp \left(\frac{\beta \alpha^{*}-\beta^{*} \alpha}{2}\right) \exp \left(-\frac{\alpha \alpha^{*}}{2}\right) \alpha^{n} \\
& =\exp \left(-\frac{\beta \beta^{*}}{2}\right) \sum_{n=0}^{\infty} \frac{\beta^{n}}{\sqrt{n !}}|n\rangle=|\beta\rangle,
\end{aligned}
$$

and in analogous way

$$
\frac{1}{2 \pi} \int \frac{\mathrm{i}}{2} \mathrm{~d} \alpha \wedge \mathrm{d} \alpha^{*}\langle\alpha| \exp \left(-\frac{\beta \alpha^{*}-\beta^{*} \alpha}{2}\right)=\langle\beta| .
$$

Due to

$$
D\left(\alpha, \alpha^{*}\right)|\beta\rangle=\exp \left(\frac{\alpha \beta^{*}-\alpha^{*} \beta}{2}\right)|\alpha+\beta\rangle
$$

one has 


$$
D\left(\alpha, \alpha^{*}\right)|\beta\rangle\left\langle-\beta\left|\left(D\left(\alpha, \alpha^{*}\right)\right)^{\dagger}=\exp \left(\alpha \beta^{*}-\alpha^{*} \beta\right)\right| \alpha+\beta\right\rangle\langle\alpha-\beta|,
$$

and the displaced parity operator $\Pi\left(\alpha, \alpha^{*}\right) \equiv D\left(\alpha, \alpha^{*}\right) \Pi\left(D\left(\alpha, \alpha^{*}\right)\right)^{\dagger}$ becomes

$$
\Pi\left(\alpha, \alpha^{*}\right)=\frac{1}{\pi} \int \frac{\mathrm{i}}{2} \mathrm{~d} \beta \wedge \mathrm{d} \beta^{*} \exp \left(\alpha \beta^{*}-\alpha^{*} \beta\right)|\alpha+\beta\rangle\langle\alpha-\beta| .
$$

This together with (6.1) leads to the representation (6.2) of the Wigner quasiprobability.

\section{Appendix B}

\section{Completeness Relation over Heisenberg-Weyl Group and Application to Displaced Number States}

From the two-dimensional integrals over the complex $z$-plane

$$
\frac{1}{\pi c^{2}} \int \frac{\mathrm{i}}{2} \mathrm{~d} z \wedge \mathrm{d} z^{*} \exp \left(-\frac{z z^{*}}{c^{2}}\right) z^{m} z^{* n}=n ! c^{2 n} \delta_{m, n}
$$

which can be calculated by transition to polar coordinates one obtains

$$
\begin{aligned}
& \frac{1}{\pi} \int \frac{\mathrm{i}}{2} \mathrm{~d} z \wedge \mathrm{d} z^{*} \exp \left(-z z^{*}\right) \mathrm{L}_{m, n}\left(z, z^{*}\right) \\
& =\sum_{j=0}^{\{m, n\}} \frac{m ! n !(-1)^{j}}{j !(m-j) !(n-j) !} \frac{1}{\pi} \int \frac{\mathrm{i}}{2} \mathrm{~d} z \wedge \mathrm{d} z^{*} \exp \left(-z z^{*}\right) z^{m-j} z^{*_{n-j}} \\
& =m ! \sum_{j=0}^{n} \frac{n !(-1)^{j}}{j !(n-j) !} \delta_{m-j, n-j}=m ! \delta_{m, n}(1-1)^{n}=n ! \delta_{m, n} \delta_{n, 0}=\delta_{m, 0} \delta_{n, 0} .
\end{aligned}
$$

Now, using the definitions (7.1) of Laguerre 2D polynomials and their differentiations (7.3) and, furthermore, partial integration we calculate the following integrals (remind $\mathrm{L}_{k, n}\left(z, z^{*}\right)=\left(\mathrm{L}_{n, k}\left(z, z^{*}\right)\right)^{*}$ )

$$
\begin{aligned}
& \frac{1}{\pi} \int \frac{\mathrm{i}}{2} \mathrm{~d} z \wedge \mathrm{d} z^{*} \exp \left(-z z^{*}\right) \mathrm{L}_{m, l}\left(z, z^{*}\right) \mathrm{L}_{k, n}\left(z, z^{*}\right) \\
& =(-1)^{k+n} \frac{1}{\pi} \int \frac{\mathrm{i}}{2} \mathrm{~d} z \wedge \mathrm{d} z^{*} \mathrm{~L}_{m, l}\left(z, z^{*}\right) \frac{\partial^{k+n}}{\partial z^{* k} \partial z^{n}} \exp \left(-z z^{*}\right) \\
& =\frac{1}{\pi} \int \frac{\mathrm{i}}{2} \mathrm{~d} z \wedge \mathrm{d} z^{*} \exp \left(-z z^{*}\right) \frac{\partial^{k+n}}{\partial z^{* k} \partial z^{n}} \mathrm{~L}_{m, l}\left(z, z^{*}\right) \\
& =\frac{1}{\pi} \int \frac{\mathrm{i}}{2} \mathrm{~d} z \wedge \mathrm{d} z^{*} \exp \left(-z z^{*}\right) \frac{m ! l !}{(m-n) !(l-k) !} \mathrm{L}_{m-n, l-k}\left(z, z^{*}\right) \\
& =m ! l ! \delta_{m-n, 0} \delta_{l-k, 0}=m ! l ! \delta_{m, n} \delta_{k, l} .
\end{aligned}
$$

From this follows using the decomposition (7.6) of displaced number states into number states

$$
\begin{aligned}
& \frac{1}{\pi} \int \frac{\mathrm{i}}{2} \mathrm{~d} \alpha \wedge \mathrm{d} \alpha^{*} D\left(\alpha, \alpha^{*}\right)|n\rangle\left\langle m\left|\left(D\left(\alpha, \alpha^{*}\right)\right)^{\dagger}=\frac{1}{\pi} \int \frac{\mathrm{i}}{2} \mathrm{~d} \alpha \wedge \mathrm{d} \alpha^{*}\right| \alpha, n\right\rangle\langle\alpha, m| \\
& =\frac{(-1)^{m+n}}{\sqrt{m ! n !}} m ! \delta_{m, n} \sum_{k=0}^{\infty} \sum_{l=0}^{\infty} \frac{|k\rangle\langle l|}{\sqrt{k ! l !}} \frac{1}{\pi} \int \frac{\mathrm{i}}{2} \mathrm{~d} \alpha \wedge \mathrm{d} \alpha^{*} \exp \left(-\alpha \alpha^{*}\right) \mathrm{L}_{m, l}\left(\alpha, \alpha^{*}\right) \mathrm{L}_{k, n}\left(\alpha, \alpha^{*}\right) \\
& =\delta_{m, n} \sum_{k=0}^{\infty} \sum_{l=0}^{\infty} \frac{|k\rangle\langle l|}{\sqrt{k ! l !}} l ! \delta_{k, l}=\langle m \mid n\rangle \sum_{l=0}^{\infty}|l\rangle\langle l|=\langle m \mid n\rangle I .
\end{aligned}
$$


For an arbitrary operator $A$ follows from this relation

$$
\begin{aligned}
& \frac{1}{\pi} \int \frac{\mathrm{i}}{2} \mathrm{~d} \alpha \wedge \mathrm{d} \alpha^{*} D\left(\alpha, \alpha^{*}\right) A\left(D\left(\alpha, \alpha^{*}\right)\right)^{\dagger} \\
& =\sum_{m=0}^{\infty} \sum_{n=0}^{\infty} \frac{1}{\pi} \int \frac{\mathrm{i}}{2} \mathrm{~d} \alpha \wedge \mathrm{d} \alpha^{*} D\left(\alpha, \alpha^{*}\right)|n\rangle\langle n|A| m\rangle\langle m|\left(D\left(\alpha, \alpha^{*}\right)\right)^{\dagger} \\
& =\sum_{m=0}^{\infty} \sum_{n=0}^{\infty}\langle n|A| m\rangle \frac{1}{\pi} \int \frac{\mathrm{i}}{2} \mathrm{~d} \alpha \wedge \mathrm{d} \alpha^{*}|\alpha, n\rangle\langle\alpha, m| \\
& =\sum_{m=0}^{\infty} \sum_{n=0}^{\infty}\langle n|A| m\rangle\langle m \mid n\rangle I=\langle A\rangle I .
\end{aligned}
$$

This is the (over-)completeness integral (5.14) for displaced operators $A$ and follows also from general orthogonality relations of group representations if one knows the invariant measure over the Lie group.

In an analogous way using the number representation of the coherent states one calculates

$$
\begin{aligned}
& \frac{1}{2 \pi} \int \frac{\mathrm{i}}{2} \mathrm{~d} \alpha \wedge \mathrm{d} \alpha^{*} D\left(\alpha, \alpha^{*}\right) \\
& =\sum_{m=0}^{\infty}|m\rangle\left\langle m\left|\left(\frac{1}{2 \pi} \int \frac{\mathrm{i}}{2} \mathrm{~d} \alpha \wedge \mathrm{d} \alpha^{*} \exp \left(-\frac{\alpha \alpha^{*}}{2}\right) \exp \left(\alpha a^{\dagger}\right) \exp \left(-\alpha^{*} a\right)\right) \sum_{n=0}^{\infty}\right| n\right\rangle\langle n| \\
& =\sum_{m=0}^{\infty} \sum_{n=0}^{\infty} \frac{|m\rangle\langle n|}{\sqrt{m ! n !}} \sum_{j=0}^{\{m,} \frac{(-1)^{n-j} m ! n !}{j !(m-j) !(n-j) !} \frac{1}{2 \pi} \int \frac{\mathrm{i}}{2} \mathrm{~d} \alpha \wedge \mathrm{d} \alpha^{*} \exp \left(-\frac{\alpha \alpha^{*}}{2}\right) \alpha^{m-j} \alpha^{*_{n-j}} \\
& =\sum_{n=0}^{\infty}|n\rangle\left\langle n\left|\sum_{j=0}^{n} \frac{n !(-2)^{n-j}}{j !(n-j) !}=\sum_{n=0}^{\infty}(-1)^{n}\right| n\right\rangle\langle n|=\Pi,
\end{aligned}
$$

where we used the evaluation of the integral in (B.1).

\section{Appendix C}

\section{Wigner Quasiprobability $W\left(\alpha, \alpha^{*}\right)$ from Husimi-Kano Quasiprobability $Q\left(\alpha, \alpha^{*}\right)$ via Bargmann Representation}

The Wigner quasiprobability $W\left(\alpha, \alpha^{*}\right)$ can be obtained from the Husimi-Kano quasiprobability $Q\left(\alpha, \alpha^{*}\right)$ by the relation

$$
\begin{aligned}
& W\left(\alpha, \alpha^{*}\right)=\exp \left(-\frac{1}{2} \frac{\partial^{2}}{\partial \alpha \partial \alpha^{*}}\right) Q\left(\alpha, \alpha^{*}\right), \\
& \Leftrightarrow Q\left(\alpha, \alpha^{*}\right)=\exp \left(\frac{1}{2} \frac{\partial^{2}}{\partial \alpha \partial \alpha^{*}}\right) W\left(\alpha, \alpha^{*}\right) .
\end{aligned}
$$

We introduce now in $Q\left(\alpha, \alpha^{*}\right)$ the Bargmann representation $\psi^{*}(\alpha)$ of a pure state $|\psi\rangle$ by an analytic function of the complex variable $\alpha$

$$
\begin{aligned}
& \psi^{*}(\alpha) \equiv\langle\psi \| \alpha\rangle=\sum_{n=0}^{\infty}\langle\psi \mid n\rangle \frac{\alpha^{n}}{\sqrt{n !}}, \\
& \psi\left(\alpha^{*}\right) \equiv\langle\alpha \| \psi\rangle=\sum_{n=0}^{\infty}\langle n \mid \psi\rangle \frac{\alpha^{* n}}{\sqrt{n !}}=\left(\psi^{*}(\alpha)\right)^{*},
\end{aligned}
$$

where $\| \alpha\rangle=\exp \left(\frac{\alpha \alpha^{*}}{2}\right)|\alpha\rangle$ are the analytic (non-normalized) coherent states. 
Then $Q\left(\alpha, \alpha^{*}\right)$ is

$$
Q\left(\alpha, \alpha^{*}\right)=\frac{1}{\pi} \exp \left(-\alpha \alpha^{*}\right) \psi^{*}(\alpha) \psi\left(\alpha^{*}\right),
$$

and the Wigner quasiprobability (C.1) becomes

$$
W\left(\alpha, \alpha^{*}\right)=\frac{1}{\pi} \exp \left(-\frac{1}{2} \frac{\partial^{2}}{\partial \alpha \partial \alpha^{*}}\right) \exp \left(-\alpha \alpha^{*}\right) \psi^{*}(\alpha) \psi\left(\alpha^{*}\right) .
$$

We will give this formula another principal form using the Lie group $S U(1,1)$. This may be useful for similar considerations to more general quasiprobabilities.

Introducing the operators

$$
K_{-} \equiv \frac{\partial^{2}}{\partial \alpha \partial \alpha^{*}}, \quad K_{+} \equiv \alpha \alpha^{*}, \quad K_{0} \equiv \frac{1}{2}\left(\alpha \frac{\partial}{\partial \alpha}+\alpha^{*} \frac{\partial}{\partial \alpha^{*}}+1\right),
$$

one finds that they satisfy the commutation relations (9.3) and, therefore, form a realization of the Lie algebra $s u(1,1)$ to the Lie group $S U(1,1)$. The corresponding Casimir operator $C$ is

$$
C \equiv\left(K_{0}\right)^{2}-\frac{1}{2}\left(K_{-} K_{+}+K_{+} K_{-}\right)=\frac{1}{4}\left\{\left(\alpha \frac{\partial}{\partial \alpha}-\alpha^{*} \frac{\partial}{\partial \alpha^{*}}\right)^{2}-1\right\} .
$$

Using the commutation relations

$\exp \left(\alpha \alpha^{*}\right) \frac{\partial^{2}}{\partial \alpha \partial \alpha^{*}} \exp \left(-\alpha \alpha^{*}\right)=\left(\frac{\partial}{\partial \alpha}-\alpha^{*}\right)\left(\frac{\partial}{\partial \alpha^{*}}-\alpha\right)$ one may transforms (C.4) to the form

$$
W\left(\alpha, \alpha^{*}\right)=\frac{1}{\pi} \exp \left(-\alpha \alpha^{*}\right) \exp \left\{-\frac{1}{2}\left(\frac{\partial}{\partial \alpha}-\alpha^{*}\right)\left(\frac{\partial}{\partial \alpha^{*}}-\alpha\right)\right\} \psi^{*}(\alpha) \psi\left(\alpha^{*}\right) .
$$

The entangled operator $\exp \left\{-\frac{1}{2}\left(\frac{\partial}{\partial \alpha}-\alpha^{*}\right)\left(\frac{\partial}{\partial \alpha^{*}}-\alpha\right)\right\}$ of $S U(1,1)$ can be disentangled using the relations in [37] (Appendix A, Equation (A.14)) according to

$$
\begin{aligned}
& \exp \left\{-\frac{1}{2}\left(\frac{\partial}{\partial \alpha}-\alpha^{*}\right)\left(\frac{\partial}{\partial \alpha^{*}}-\alpha\right)\right\} \\
& =\exp \left\{-\frac{1}{2}\left(K_{-}-2 K_{0}+K_{+}\right)\right\}=\exp \left(-K_{+}\right) \exp \left(-\frac{1}{4} K_{-}\right) 2^{2 K_{0}} \\
& =\exp \left(-\alpha \alpha^{*}\right) \exp \left(-\frac{1}{4} \frac{\partial^{2}}{\partial \alpha \partial \alpha^{*}}\right) 2^{\alpha \frac{\partial}{\partial \alpha}+\alpha^{*} \frac{\partial}{\partial \alpha^{*}+1}}
\end{aligned}
$$

belonging to the unimodular matrix $\left(\begin{array}{cc}\kappa & \lambda \\ \mu & v\end{array}\right)=\frac{1}{2}\left(\begin{array}{cc}1 & -1 \\ 1 & 3\end{array}\right)$ with $\varepsilon=0 \quad$ (see [37],

Equation (A.7)). This leads to the representation of (C.7)

$W\left(\alpha, \alpha^{*}\right)=\frac{1}{\pi} \exp (-\alpha \alpha)\left\{\exp \left(-\alpha \alpha^{*}\right) \exp \left(-\frac{1}{4} \frac{\partial^{2}}{\partial \alpha \partial \alpha^{*}}\right) 2^{\alpha \frac{\partial}{\partial \alpha}+\alpha^{*} \frac{\partial}{\partial \alpha^{*}+1}}\right\} \psi^{*}(\alpha) \psi\left(\alpha^{*}\right)$. 
The applied technique is a special case $(s=-1 / 2, r=1)$ of the technique applied in [11] (Appendix $\mathbf{A}$ there) and the operator $2^{\alpha \frac{\partial}{\partial \alpha}+\alpha^{*} \frac{\partial}{\partial \alpha^{*}}}$ is the operator of multiplication of both arguments $\left(\alpha, \alpha^{*}\right)$ of an arbitrary function $f\left(\alpha, \alpha^{*}\right)$ by the factor 2 . This leads to the new representation of the Wigner quasiprobability

$$
\begin{aligned}
W\left(\alpha, \alpha^{*}\right) & =\frac{2}{\pi} \exp (-2 \alpha \alpha) \exp \left(-\frac{1}{4} \frac{\partial^{2}}{\partial \alpha \partial \alpha^{*}}\right) \psi^{*}(2 \alpha) \psi\left(2 \alpha^{*}\right) \\
& =2 \exp (-2 \alpha \alpha) \exp \left(-\frac{1}{4} \frac{\partial^{2}}{\partial \alpha \partial \alpha^{*}}\right) \exp \left(4 \alpha \alpha^{*}\right) Q\left(2 \alpha, 2 \alpha^{*}\right)
\end{aligned}
$$

With the definition of Laguerre 2D polynomials $\mathrm{L}_{m, n}\left(z, z^{*}\right)$ in (7.1) and using (C.2) this is equivalent to

$$
W\left(\alpha, \alpha^{*}\right)=\frac{2}{\pi} \exp \left(-2 \alpha \alpha^{*}\right) \sum_{m=0}^{\infty} \sum_{n=0}^{\infty} \frac{\langle n \mid \psi\rangle\langle\psi \mid m\rangle}{\sqrt{m ! n !}} \mathrm{L}_{m, n}\left(2 \alpha, 2 \alpha^{*}\right) .
$$

The same relation was derived in other way in [23] (Equation (5.16)) and it was known also without using Laguerre 2D polynomials.

\section{Appendix D}

About the Unorthodox Entire Function $f(z) \equiv \sum_{n=0}^{\infty} \frac{z^{n}}{\sqrt{n !}}$

We make here some remarks about the entire function ${ }^{9}$

$$
f(z) \equiv \sum_{n=0}^{\infty} \frac{z^{n}}{\sqrt{n !}}=\sum_{m=0}^{\infty} \frac{z^{2 m}}{\sqrt{(2 m) !}}+\sum_{m=0}^{\infty} \frac{z^{2 m+1}}{\sqrt{(2 m+1)}} .
$$

playing a main role in calculations with coherent phase states and which is an unorthodox function for which it is difficult to obtain usable relations.

Using the well-known doubling formula of the argument of a Gamma function one may represent (D.1) with introduction of a new function $f_{0}(z)$ as follows $\left(\left(-\frac{1}{2}\right) !=\sqrt{\pi}\right)$

$$
\begin{aligned}
f(z) & =\sum_{m=0}^{\infty} \frac{1}{m !}\left(\frac{z^{2}}{2}\right)^{m}\left(\sqrt{\frac{m !\left(-\frac{1}{2}\right)}{\left(m-\frac{1}{2}\right)}}+z \sqrt{\frac{m !\left(+\frac{1}{2}\right) !}{\left(m+\frac{1}{2}\right) !}}\right) \\
& \equiv \exp \left(\frac{z^{2}}{2}\right) f_{01}(z) .
\end{aligned}
$$

with limiting case of the product of coefficients

${ }^{9}$ The more general function $\sum_{n=0}^{\infty} \frac{z^{n}}{(n !)^{\alpha}}$ is shortly considered in the monograph of Titchmarsh [36] on p. 255 and it is shown that their order (of growth) is $1 / \alpha$ that means 2 in our case in accordance with (D.2). 


$$
\lim _{m \rightarrow \infty} \sqrt{\frac{m !\left(-\frac{1}{2}\right)}{\left(m-\frac{1}{2}\right)}} \sqrt[!]{\frac{m !\left(+\frac{1}{2}\right)}{\left(m+\frac{1}{2}\right)}}=\sqrt{\frac{\pi}{2}}=1.253314 .
$$

The function $f(x)$ increases very rapidly for $x \rightarrow+\infty$ and from (D.2) it can be seen that it is favorable to split from $f(z)$ a function $\exp \left(\frac{z^{2}}{2}\right)$ in multiplicative way (in accordance with the order of the function) and to consider the remaining function $f_{0}(z)$ which for $x \rightarrow+\infty$ does not increase rapidly such as $f(x)$ and for $x \rightarrow-\infty$ does not vanish so rapidly as $f(x)$.

To give an impression of the function $f_{0}(z)$ we write down the first 6 sum terms of its Taylor series in analytic and in numerical representation

$$
\begin{aligned}
f_{0}(z)= & 1+z+\frac{1}{2}(-1+\sqrt{2}) z^{2}+\frac{1}{6}(-3+\sqrt{6}) z^{3}+\frac{1}{24}(3-6 \sqrt{2}+2 \sqrt{6}) z^{4} \\
& +\frac{1}{120}(15-10 \sqrt{6}+2 \sqrt{30}) z^{5}+\frac{1}{240}(-5+15 \sqrt{2}+4 \sqrt{5}-10 \sqrt{6}) z^{6}+\cdots \\
\approx & 1+z+0.207107 z^{2}-0.0917517 z^{3}-0.0244292 z^{4} \\
& +0.0121629 z^{5}+0.00276074 z^{6}+\cdots
\end{aligned}
$$

After the first constant term equal to 1 follow alternatingly two sum terms with positive and two sum terms with negative signs in its Taylor series. The zeros of the functions $f(z)$ and of $f_{0}(z)$ are the same but in front of powers $z^{n}$ we have in (D.4) coefficients with approximately $n$ sum terms involving square roots.

The derivatives of the function $f(z)$ are

$$
\frac{\partial}{\partial z} f(z)=\sum_{n=0}^{\infty} \sqrt{n+1} \frac{z^{n}}{\sqrt{n !}}, \Rightarrow \frac{\partial^{k}}{\partial z^{k}} f(z)=\sum_{n=0}^{\infty}(n+1)^{\frac{k}{2}} \frac{z^{n}}{\sqrt{n !}},
$$

or for the function $f_{0}(z)$

$$
\frac{\partial}{\partial z} f_{0}(z) \equiv \frac{\partial}{\partial z} \exp \left(-\frac{z^{2}}{2}\right) \sum_{n=0}^{\infty} \frac{z^{n}}{\sqrt{n !}}=\exp \left(-\frac{z^{2}}{2}\right) \sum_{n=0}^{\infty}(\sqrt{n+1}-z) \frac{z^{n}}{\sqrt{n !}} .
$$

The derivatives of $f(z)$ cannot be closed in finite way and lead to ever new functions of the starting type which grow weakly faster than $f(z)$ for $z=x \rightarrow+\infty$. This is one of the difficulties of treatment of the function $f(z)$.

On the real positive axis $z=x$ we obviously do not have zeros and all zeros possess a positive real part. In [32] we calculated (end of nineties) the first pairs of complex conjugate zeros which are (see footnote 10):

$$
\begin{aligned}
& z_{1}^{ \pm}=1.71697 \pm \mathrm{i} 3.18011, \quad\left|z_{1}\right|=3.61401, \quad z_{1} z_{1}^{*}=13.0611, \\
& z_{2}^{ \pm}=2.88669 \pm \mathrm{i} 4.09032, \quad\left|z_{2}\right|=5.00637, \quad z_{2} z_{2}^{*}=25.0637, \\
& z_{3}^{ \pm}=3.75716 \pm \mathrm{i} 4.81893, \quad\left|z_{3}\right|=6.11051, \quad z_{3} z_{3}^{*}=37.3383, \\
& z_{4}^{ \pm}=4.47871 \pm \mathrm{i} 5.44616, \quad\left|z_{4}\right|=7.05121, \quad z_{4} z_{4}^{*}=49.7195, \\
& z_{5}^{ \pm}=5.10(5 ?) \pm \mathrm{i} 6.00(5 ?), \quad\left|z_{5}\right|=7.88(2 ?), \quad z_{5} z_{5}^{*}=62 .(13 ?), \\
& z_{6}^{ \pm}=5.6(6 ?) \pm \mathrm{i} 6.5(1 ?), \quad\left|z_{6}\right|=8.6(3 ?), \quad z_{6} z_{6}^{*}=74 .(42 ?) \text {. }
\end{aligned}
$$


The function $f_{0}(z)$ possesses the same zeros as the function $f(z)$ and their calculation from this function is perhaps more appropriate (however, each coefficient to $z^{n}$ contains then approximately the sum of $n$ terms of square roots of integers).

It is almost evident that the function $f(z)$ does not possess zeros on the real negative axis. For this purpose one may calculate the product $f(z) f(-z)$ according to

$$
\begin{aligned}
f(-z) f(z) & =\sum_{k=0}^{\infty}(-1)^{k} \frac{z^{k}}{\sqrt{k !}} \sum_{l=0}^{\infty} \frac{z^{l}}{\sqrt{l !}}=\sum_{n=0}^{\infty} \frac{z^{n}}{\sqrt{n !}} \sum_{k=0}^{n}(-1)^{k} \sqrt{\frac{n !}{k !(n-k) !}} \\
& =\sum_{m=0}^{\infty} \frac{z^{2 m}}{\sqrt{(2 m) !}} \sum_{k=0}^{2 m}(-1)^{k} \sqrt{\frac{(2 m) !}{k !(2 m-k) !}} .
\end{aligned}
$$

To prove the absence of zeros of $f(z)$ on the negative axis (on the positive axis this is clear) it would be sufficient to prove (or more generally to prove expression in round brackets would be interesting)

$$
\sum_{k=0}^{2 m}(-1)^{k} \sqrt{\frac{(2 m) !}{k !(2 m-k) !}}>0, \quad\left(\sum_{k=0}^{2 m}(-1)^{k}\left(\frac{(2 m) !}{k !(2 m-k) !}\right)^{\alpha}>0,(0<\alpha<1) .\right.
$$

Then for real $z=x$ the product $f(-x) f(x)$ is positive in every case. Numerical calculations show "almost evidence" of this relation but a correct proof seems to be difficult and we did not find such up to now.

All this illustrates that we have a lot of difficulties when we have to work with the unorthodox entire function $f(z)$ and when we have to apply this functions within more complicated relations.

In 2008 S. Skorokhodov sent me a nice e-mail with essential passages given in the footnote ${ }^{10}$. It is a pity that with my present computer I can no more open the appended file.

\footnotetext{
${ }^{10}$ Dear ... Wuensche!

I am Skorokhodov Sergey from Computing Centre of the Russian Academy of Sciences. I have studied your paper "An unorthodox function" in Jour. of Comput. and Applied Mathem. V. $133 \mathrm{~N}$ 1-2 (2001). ... I have evaluated the first 35 zeros of the function (on a figure -- 0 ) and have derived a simple approximation to zeros on the base of combination of two hypergeometric functions ${ }_{1} F_{1}$ (on a figure in I-st quadrant -- $\mathrm{x}$ ). The error is rather small. ... I have evaluated also the zeros of the entire functions $f(\alpha ; z)=\sum_{n=0}^{\infty} \frac{z^{n}}{n !^{\alpha}}, \alpha \in(0,1)$. The distribution of zeros is a very interesting also.
} 